\title{
TRAC Code Modifications Made for APT Blanket Safety Analyses
}

by

L. L. Hamm

Westinghouse Savannah River Company

Savannah River Site

Aiken, South Carolina 29808

S. Y. Lee

M. A. Shadday

F. G. Smith, III

A document prepared for SENDING TO LANL at , , from - .

DOE Contract No. DE-AC09-96SR18500

This paper was prepared in connection with work done under the above contract number with the U.S.

Department of Energy. By acceptance of this paper, the publisher and/or recipient acknowledges the U.S. Government's right to retain a nonexclusive, royalty-free license in and to any copyright covering this paper, along with the right to reproduce and to authorize others to reproduce all or part of the copyrighted paper. 


\section{DISCLAIMER}

Portions of this document may be illegible in electronic image products. Images are produced from the best available original document. 


\section{TRAC CODE MODIFICATIONS MADE FOR APT BLANKET SAFETY ANALYSES}

L. Larry Hamm

Si Young Lee

M. Andy Shadday

Frank G. Smith, III

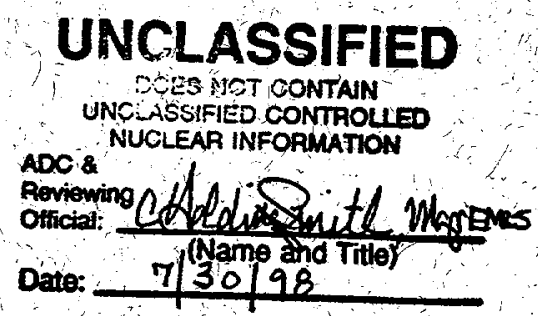

Westinghouse Savannah River Company Savannah River Site Aiken, SC 29808

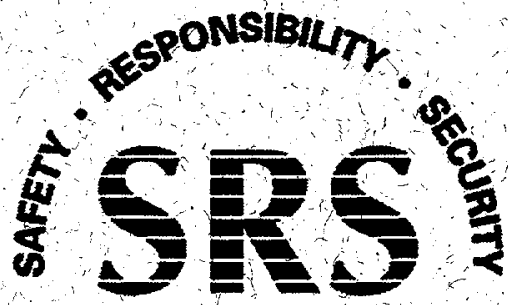




\section{DISCLAIMER}

This report was prepared as an account of work sponsored by an agency of the United States Government. Neither the United States Government nor any agency thereof, nor any of their employees, makes any warranty, express or implied, or assumes any legal liability or responsibility for the accuracy, completeness, or usefulness of any information, apparatus, product, or process disclosed, or represents that its use would not infringe privately owned rights. Reference herein to any specific commercial product, process, or service by trade name, trademark, manufacturer, or otherwise does not necessarily constitute or imply its endorsement, recommendation, or favoring by the United States Government or any agency thereof. The views and opinions of authors expressed herein do not necessarily state or reflect those of the United States Government or any agency thereof.

This report has been reproduced directly from the best available copy.

Available to DOE and DOE contractors from the Office of Scientific and Technical Information, P.O. Box 62, Oak Ridge, TN 37831; prices available from (615) 576-8401.

Available to the public from the National Technical Information Service, U.S. Department of Commerce, 5285 Port Royal Road, Springfield, VA 22161. 


\section{WSRC-TR-98-0054}

\section{KEYWORDS:}

Accelerator Production of Tritium Blanket System

Conceptual Design

TRAC Code

System Model

Safety Analysis

RETENTION - Permanent

\section{TRAC CODE MODIFICATIONS MADE FOR APT BLANKET SAFETY ANALYSES}

SAVANNAH RIVER TECHNOLOGY CENTER

L. Larry Hamm

Si Young Lee

M. Andy Shadday

Frank G. Smith, III

Publication Date: July 1998

Westinghouse Savannah River Company

Savannah River Site

Aiken, SC 29808

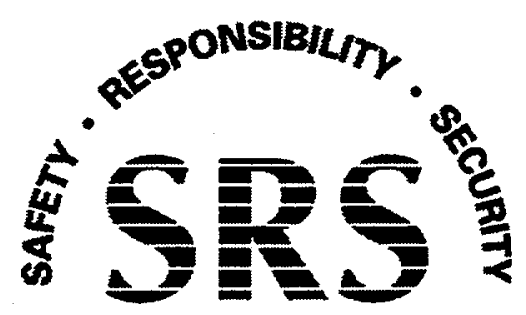

SAYANNAH RIVER SITE

Prepared for the U.S. Department of Energy under Contract No. DE-AC09-96SR18500 
DOCUMENT: WSRC-TR-98-0054

TITLE: TRAC CODE MODIFICATIONS MADE FOR APT

BLANKET SAFETY ANALYSES

APPROVALS

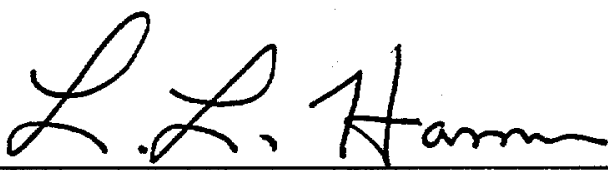

L. Larry Hamm, Task Leader (EM\&S Group/SRTC)

Date: $7-16-98$

hrgony Le

Si Young Lee, Co-author (EM\&S Group/SRTC)

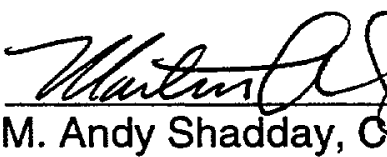

M. Andy Shadday, Colauthor (EM\&S Group/SRTC)

Trank A 1 meth II

Frank G. Smith, III, Co-author (PC\&C Group/SRTC)

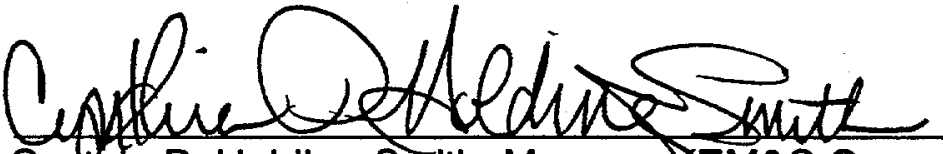

Cynthia P. Holding-Smith, Manager M \&S Group/SRTC)

m.Q. ¿ीra

Mattha A. Ebra, Manager (EDS/SRTC)

Date: $2 / 20 / 98$

Date: $7 / 20 / 98$

Date: $7-20-98$

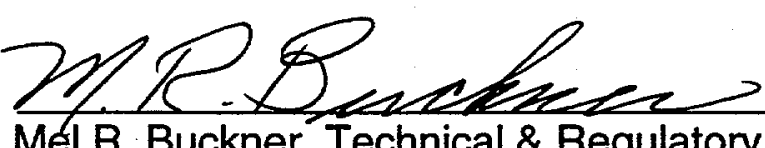

Mel R. Buckner, Technical \& Regulatory Lead (APT OPO) 


\section{Table of Contents}

1 Introduction 1

2 Background 1

3 TRAC Code $\quad 2$

3.1 TRAC Source Modifications ................................................................................. 2

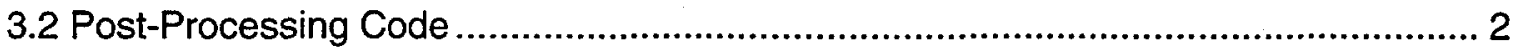

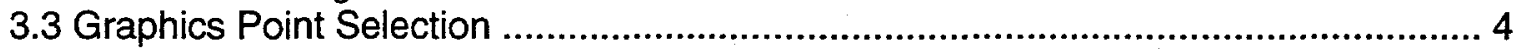

3.4 Configuration Management Control ................................................................ 4

4 Conclusions $\quad 4$

5 References $\quad 4$

Appendix A: Listing of Modified TRAC Subroutines for Graphical Output Purposes

Appendix B: Listing of Post-Processing Program TSPLIT

Appendix C: Listing of Example "graphin" File 
(This Page Intentionally Left Blank) 


\section{Introduction}

This report provides documentation of the necessary source code modifications made to the TRAC-PF1/MOD2 code version 5.4.28a [1] developed at Los Alamos National Laboratory (LANL). Functionally, the code modifications impact only a relatively small number of the FORTRAN subroutines and have no effect on the physical models or numerics contained within this version of TRAC. These modifications add one additional output file to a TRAC run in the form of an ASCll graphical data set. Verification testing was performed to ensure that no unwanted side effects resulted from the source code modifications. The modifications made are independent of computing platform and were tested on both IBM RS/6000 580's and 590's, and the SRS CRAY J90 computing machines.

\section{Background}

The original TRAC code has built-in output features in the form of: (1) an ASCII printout of results tabulated on a component-by-component basis; and (2) graphics files where post-processing using special purpose LANL algorithms are employed. Within the Savannah River Technology Center (SRTC), personnel have a great deal of experience using the commercially available software package named "TecPlot' ${ }^{\mathrm{TM}}$ " [2]. TecPlot ${ }^{\mathrm{TM}}$ is a widely used scientific and engineering data visualization software package for interactively creating multi-dimensional graphics. Based on scheduling, training, and purchasing considerations, it was determined that implementing minor source code modifications to TRAC in order to make it TecPlot ${ }^{\text {TM }}$ compatible would be more expedient than pursuing the special purpose software that already existed at LANL for TRAC.

The modifications were made to the standard output routines in order to generate an ASCII output file called "tecsum.grf". This output file contains results at each requested graphics point (i.e. TRAC component and specified cell number) written out at a specified time frequency. In a post-processing mode, the ASCII graphic output file is split into individual ASCII data set files for each graphics point using a special purpose FORTRAN program named "TSPLIT". The output from TSPLIT was formatted to be TecPlot ${ }^{\mathrm{T} M}$ ready.

Implementation and verification testing was performed on TRAC and TSPLIT to ascertain that no unwanted sides effects had been incorporated into TRAC and that correct graphical data sets were being produced. Identical test problems were run using both the original TRAC code and its modified version. The resulting "trcout" printed output files were then compared for potential differences. The only differences observed were those associated with the writing of a notice to the user that a "tecsum.grf" file had been created. Also, the results from the post-processing of the "tecsum.grf" file using TSPLIT were compared with those contained directly within the "trcout" file. No errors were identified in either test. 


\section{TRAC Code}

\subsection{TRAC Source Modifications}

The following is a listing of the TRAC subroutines affected by the above source code modifications:

$\begin{array}{llll}\text { clean.f } & \text { ecomp.f } & \text { wbreak.f } & \text { wcomp.f } \\ \text { wfill.f } & \text { whtstr.f } & \text { wpipe.f } & \text { wplen.f } \\ \text { wprizr.f } & \text { wpump.f } & \text { wtee.f } & \text { wturb.f } \\ \text { wvlve.f } & & & \end{array}$

A listing of each FORTRAN subroutine is provide in Appendix A. Note that limitations exist within the current set of modifications. Due to TRAC's staggered mesh (i.e. state variables at cell centers and kinematic variables. at cell faces) and the need to donor properties across cell faces, special logic is required to locate incoming material into a component's edge from its neighboring component. The current modifications do not handle this condition properly since no attempt is made to locate potential neighboring components. As a result, certain quantities at cell faces associated with a component's ends may not be correctly computed. This will occur for incoming material flowing into a given component and when the graphics point selected corresponds to that component's first or last cell, respectively. At present, this limitation is handled by only looking at those cell's where incoming material crossing that cell is contained within the existing component. By avoiding such edges, the Blanket Safety Analyses performed were not impacted. Future upgrades can eliminate such limitations.

\subsection{Post-Processing Code}

As stated above, a special purpose FORTRAN program was written to split out the "tecsum.grf" file generated at the end of a TRAC run. The code was named "TSPLIT" and a source listing of the program is provided in Appendix B. The graphics points selected are read into TRAC from the file named "graphin". This same file is also read into TSPLIT along with the "tecsum.grf" file. TSPLIT then splits the graphics dataset file into the various graphics files associated with each specified graphics point.

The program "tsplit" takes as input the TRAC code generates and writes out to the "tecsum.grf" file and splits it out into separate "tecplot" compatible files consistent with the requested graphics points inputted to TRAC through the "graphin" file. First the data within the "tecsum.grf" file is read in and stored within appropriate arrays consistent with component type.

Then, each individual component graph point data set is written to its own unique file:

- "tecNNN-XX.dat" files for graphics points in components that are not plenums or heat structures.

- "tecp $N N N-X X$.dat" files for graphics points in plenum components.

- "tecphiNNN-XX.dat" files for graphics points on inner surfaces of heat structures.

- "tecphoNNN-XX.dat" files for graphics points on outer surfaces of heat structures.

Where NNN - represents TRAC component number (0 to 999) and $X X$ - represents cell location within component (1-99). 
The following is a listing of the variables employed in TSPLIT and a brief description of each, including units assumed:

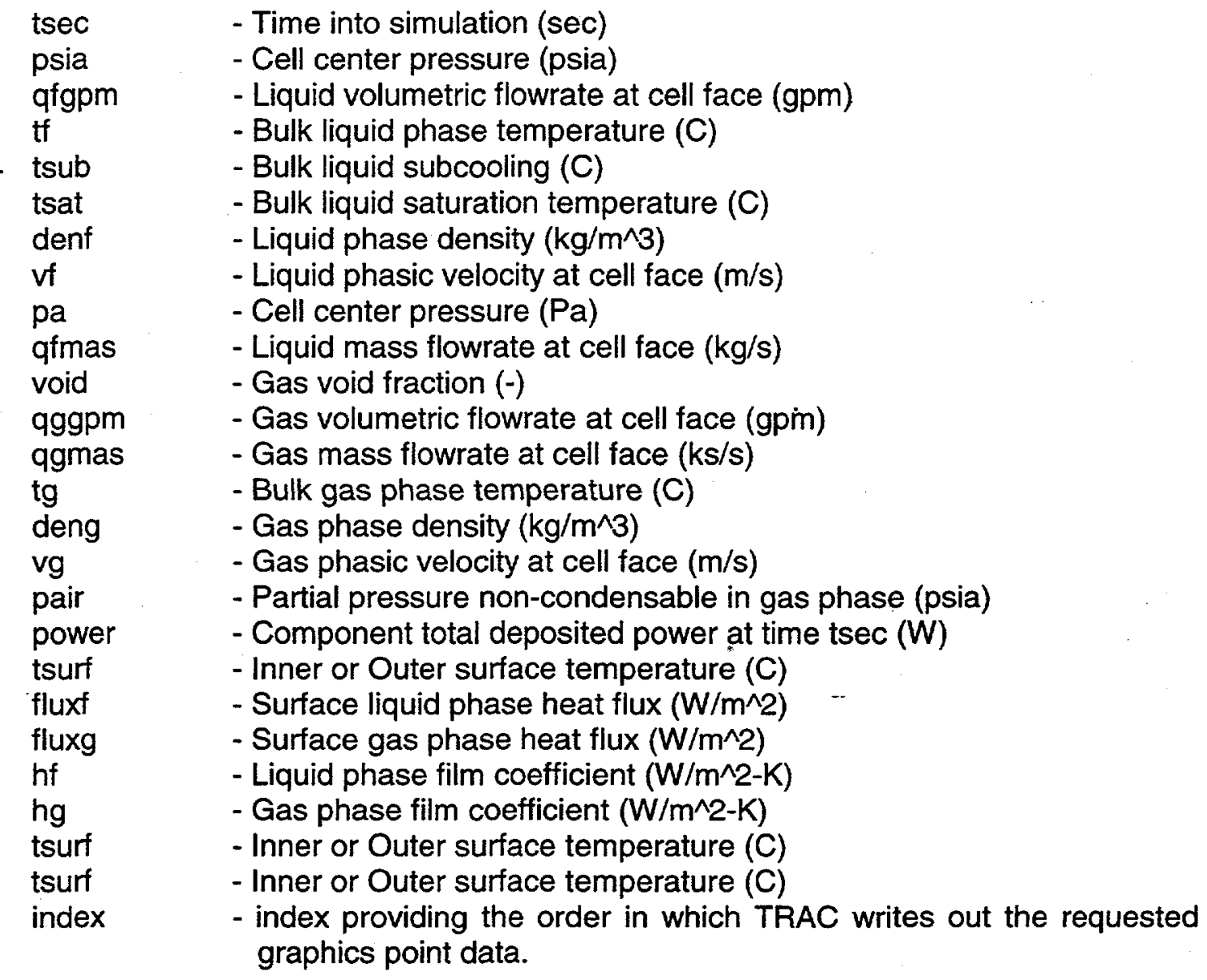




\subsection{Graphics Point Selection}

Appendix C contains an example of a typical "graphin" file used during Blanket System accident analysis runs with TRAC. The basic input to a "graphin" file consists of:

npoints - number of locations (points) within TRAC model where graphics requested.

component - component id number containing specified graph point.

cell

- cell number within component where graphics requested (number second leg of tee sequentially from end cell number on first leg) (If a heat structure then cell number is axial row of nodes where point resides).

ictype - type of component: (0 for fill, pipe, pressurizer, pump, turb, value); ( 1 for plenum); ( 2 for tee); or ( 3 for heat structure).

id_leg - which leg of component cell is located in: (1 always for fill, pipe, pressurizer, pump, plenum, turb, value); ( 1 or 2 for tee's); (for a heat structure 1 implies inner surface and 2 for outer surface).

\subsection{Configuration Management Control}

Two separate versions of TRAC are being used: (1) an IBM RS/6000 compatible version; and (2) a CRAY J90 compatible version. Separate makefile's were necessary. The modified subroutines presented above are identical on each platform and only one version of the TSPLIT program was used. The TSPLIT executable was created and run on SGI Indy's and Indigo's. Test runs have been made and verified on both platforms. Currently, the source codes are stored on the SRS 'AFS' (Andrew File System). These files will be stored and protected from modification until placed under specific software configuration control.

\section{Conclusions}

The choice to modify TRAC in order to accommodate the commercial graphics package Tecplot ${ }^{\mathrm{TM}}$ has been very successful - due to the relatively short time required to make the code modifications and the flexibility obtained in viewing TRAC results. Future upgrades will be performed as time permits.

\section{References}

1. Safety Code Development Group, "TRAC-PF1/MOD2: An Advanced Best Estimate Computer Program for Pressurized Water Reactor Thermal-Hydraulic Analysis," Los Alamos National Laboratory report LA-12031-M, Vol. 1 (NUREG/CR-5673), (July 21, 1993).

2. "Tecplot User's Manual - Version 7," Amtec Engineering, Inc., Bellevue, Washington, August 1996. 


\section{Appendix A: Listing of Modified TRAC Subroutines for Graphical Output Purposes}

The files listed below represents the entire source code modifications made to the TRAC-P code.

\section{Fortran file clean.f:}

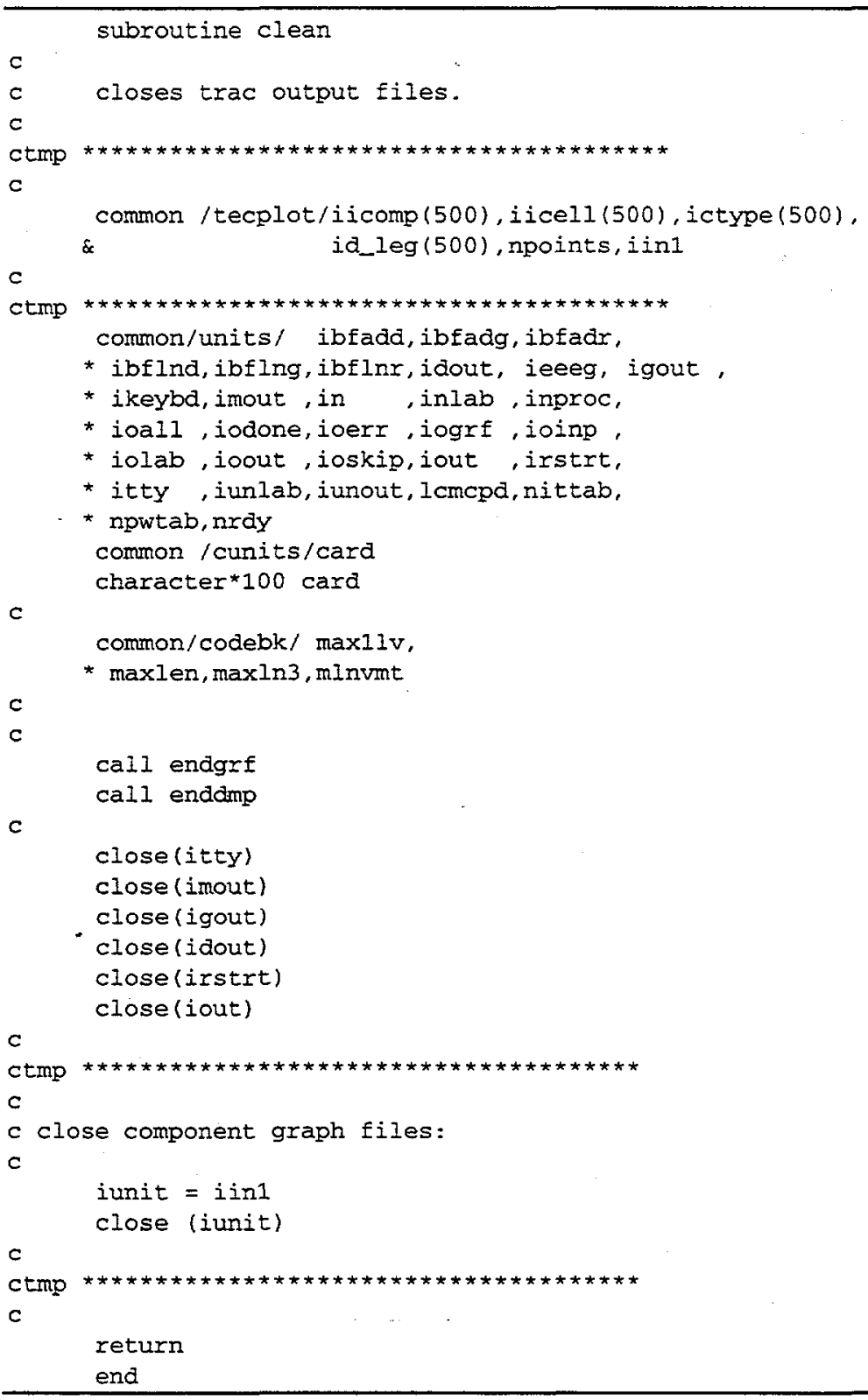

\section{Fortran file ecomp.f:}

subroutine ecomp (num, jstrt, jstop, iflgw, nodes, cfmass, xpintg,

cener, tsec, itee)

c

c subroutine ecomp writes one-dimensional component 
c

thermal-hydraulic solution parameters to file trcout

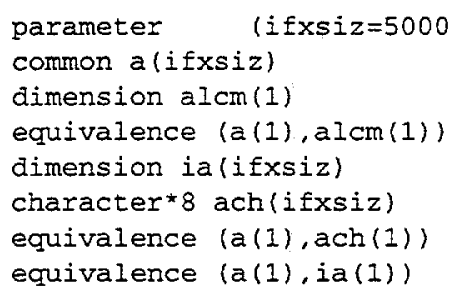

c

common/contrl/ cpuflg, damme, damx , delt,

* deltht, difmin, dprmx ,dtlmx ,dtmax ,

* atmin ,dto ,atrat ,atrmx , dtsmx ,

* atvax ,encmax, eps1 , eps2 ,epso ,

* ercemx, epss ,etime ,htlosi,htloso,

* odelt,powerc,pssmn ,pssmx , rfat

* rymax , tend , tercmx, timec , timet

* tmmax , varer, vcmn , vcrux , vmaxo,

* vmaxt, vmaxt3, vmcon, vmnew, vmold,

* vmxt3o,xtable, x0sm, yosm, $20 \mathrm{sm}$,

* omsasm,wsasm , wasasm, tosm (3), xvsm ,

* yvsm, zvsm, rmatsm $(3,3\}, \operatorname{Omsm}(3), \operatorname{wsm}(3)$,

* wdsm (3), errsm, dtsm

common/contrl/ dstep, iadded,iblaus, iccmx ,

* icmp , icmpmx, icp ,idiag, idiags, ieccpx, ieos, iff3d ,

* ifprep, igeom 3 , im100, im100x, imfr

* invan, iofftk, ipak , ipak3a, ipakon, ipkpmp,

* ireset, irsflg, irstfl, isolut, issflg, istdy .

* isttc, ithd, itmin, itpako,jfat, kccmx .

* lcmptr, levstg, llvflg, ncmn , ncmx

* ncontr, nconts, ncontt, ncrg ,ndial ,nencl,

* newrfa, nfrc1, nfrc3, nitav , nitmn,

* nitmx , nloopp, nosets, nsend ,nseo ,

* nsmn , nsmx ,nspl ,nspu ,nsso,

* nstab, nstp , nvgrav, nvpow , oitmax, sitmax,

* stayst, transi, motsm, statsm, saxsm

integer dstep, oitmax, sitmax, stdyst,

* transi, statsm, saxsm

$c$

dimension $1 d(3), \operatorname{ldn}(3)$

common/ptab/ lalp, lalpn, lalpd,

* lalpdn, lalv , lalvn , lalve , lalven,

* lara , laran, larel , lareln, larev,

* larevn, larl, larln, larv , larvn,

* Ibit , lbitn, Ichti, Ichtin, lchtia,

* lchtan, lcif, lcifn, lcone, lconen,

* la , lan, lea, lean, lel

* leln, lev, levn, Igam, Igamn,

* Inig, lhigo, Ihil ,Ihilo, Ihiv

* Inivo, lp , Ipn , Ipa ,Ipan ,

* Iqppc, Iqppco, Iroa, Iroar , Irol,

* Iroln, irov, Irovn, is , Isn

* Itce, Itcen, Ita , Itan, Iti

* Itln, ltv , Itvn , Itw , Itwn

* Itwa, Itwan, Itwe , Itwen, Ivl , Ivln.

* Ivlt, Ivlto, lvm , lvmn , Ivv,

* Ivrn , Ivvt , Ivvto

$c$ if the list of pointers is increased, must also increase (if

c necessary) kpttmp in subroutine aicomp such that kpttmp is greater

c than or equal to the total number of pointers defined in historian 
1 ils, ilu, ilun, iold

common/labelv/labels (777), labsv $(2,105)$, labun (150),

1

common/labelv/alpbet, Iuar, lucp, Iud, ludh, lue, Iuen,

1

2

3

\section{character*2}

character $* 3$

character $* 4$

character*5

character*6

character*7

character*8 labels, labun, Iuid, Iur, lusp, lusz, lutm

character*9 luha

character*10 lucp

character*11 luph

character*12 luhx, lutc, runcb

character*13 luh, luncb, Iupcb

character*14 labsv

character* 19 ludh

character* 26 alpbet

$\mathrm{c}$

ctmp

c

common /tecplot/iicomp (500), iicell (500), ictype (500),

$\&$ id_leg $(500)$, npoints, iinl

c

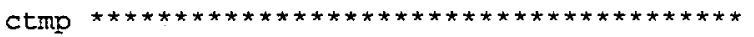

c

common/twostp/ npsfe, npsme ,ntsprn

$c$

dimension $\operatorname{tmp}(10,24)$

integer getbit

$c$

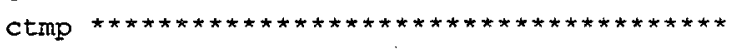

c

Conv_p $=14.696 / 101325.0$

conv_t $=273.15$

conv_a $=264.17 * 60.0$

c

ctmp

c

c

c

print out hydraulic-solution parameters

write (iout, 100) Iup, Iup, Iud, lutp, Iutp, lutp, Iur, Iur, luv, luv, Iud

100 format (/19x, 'ncd-gas'/7x, 'pressure pressure void fr. temp',

1 '.sat. temp.liq. temp.gas den.liq. den.vap. vel.liq',

2 vel.gas wf.liq.' $/$ cell' $, 3 x, a, 7 x, a, 7 x, a, 8 x, a, 8 x, a$,

$38 x, a, 5 x, a, 2 x, a, 5 x, a, 6 x, a, 6 x, a)$

$\mathrm{nn}=1+(j$ stop-jstrt $) / 10$

$j n=0$

do $130 \mathrm{n}=1, \mathrm{nn}$

$j 1=j \operatorname{strt}+(n-1) * 10$

$j 2=\min 0(j 1+9, j$ stop $)$

$j 0=j 1-1$

$j 3=j 2-j 0$

do $110 j=j 1, j 2$

$j j=j-j 0$

$j m 1=j-1$

$\operatorname{tmp}(j j, 1)=a(1 p n+j m 1)$

$\operatorname{tmp}(j j, 2)=a(1$ pan $+j m 1)$ 
$\operatorname{tmp}(j j, 3)=a(\operatorname{lalpn+jm1)}$

$\operatorname{tmp}(j j, 4)=a(1$ tsat $+j m 1)$

$\operatorname{tmp}(j j, 5)=a(1 t \ln +j m 1)$

$\operatorname{tmp}(j j, 6)=a(1 \operatorname{tvn}+j m 1)$

$\operatorname{tmp}(j j, 7)=a(1 \operatorname{roln}+j m 1)$

$\operatorname{tmp}(j j, 8)=a(1$ rovn $+j m 1)$

$\operatorname{tmp}(j j, 9)=a(1 v \ln +j m I)$

$\operatorname{tmp}(j j, 10)=a(1 \mathrm{vvn}+j m 1)$

if (getbit $\{a(1 b i t n+j m 1), 33) \cdot$ ne.0) a $(1 w f 1+j m 1)=-1.111 e-11$

$\operatorname{tmp}(j j, 11)=a(1 w f I+j m I)$

cfmass $=c$ fmass $+a(1 \mathrm{vol}+j m 1) *(a(\operatorname{larv}+j m l)+a(\operatorname{larl}+j m l))$

cener $=$ cener $+a(1 \mathrm{Vo} 1+j m 1) *(a(1$ arev $+j m 1)+a(1 \operatorname{are} l+j m 1))$

110 continue

if (ioout.eq.I) then

call uncnvt('pn', $\operatorname{tmp}(1,1), j 3,1,-1)$

call uncnvt('pan' , tmp $(1,2), j 3,1,-1)$

call uncnvt('tsat', $\operatorname{tmp}(1,4), j 3,1,-1)$

call uncnvt('tln', $\operatorname{tmp}(1,5), j 3,1,-1)$

call uncnvt('tvn', $\operatorname{tmp}(1,6), j 3,1,-1)$

call uncnvt ('roln', $\operatorname{tmp}(1,7), j 3,1,-1)$

call uncnvt ('rovn', $\operatorname{tmp}(1,8), j 3,1,-1)$

call uncnvt ('vln', tmp $\{1,9\}, j 3,1,-1)$

call uncrvt ('vvn' , $t m p(1,10), j 3,1,-1)$

endif

$j 1=j 1-j 0$

$j 2=j 2-j 0$

write (iout, 120$)(j n+j,(\operatorname{tmp}(j, k), k=1,11), j=j 1, j 2)$

120 format $(1 \mathrm{x}, \mathrm{i3}, 1 \mathrm{p}, 2 \mathrm{e} 12.5,6 \mathrm{e} 10.3,2 \mathrm{e} 11.3, \mathrm{e} 10.3)$

$j n=j n+10$

$\operatorname{ctmp}$

c Write to tecplot file data for component point if requested

$c$ and component not a plenum component or heat structure.

$c$

c Check for tee component and if a tee check that requested

$c$ cell is in first or second leg.

c

iunit $=i \operatorname{in} 1$

do ipt $=1$, npoints

jcell = iicell $(i p t)$

if ((ictype(ipt) .eq. 0 .and. itee.eq. 0 )

$\&$ or

$\& \quad$ (ictype $(i p t) . e q .2$.and. itee.eq. 1 .and.

$\& \quad$ id_leg(ipt).eq.1)

or.

(ictype(ipt).eq.2 .and. itee.eq. 2 .and.

id_leg (ipt) .eq.2)

) then

icomp $=i$ icomp $(i p t)$

if (icomp.eq. num) then

ngrps $=1+($ jcell - jstrt $) / 10$

if (ngrps.eq.n) then

$10 c=(j$ cell $-j$ strt +1$)-10 *($ ngrps -1$)$

c Compute phasic volumetric and mass flowrates.

c

void $=\operatorname{tmp}(10 \mathrm{c}, 3)$

denf $=\operatorname{tmp}(10 \mathrm{c}, 7)$

if (jcell.gt.jstrt and. $\operatorname{tmp}(10 \mathrm{c}, 9) . g e .0 .0)$ then

void $=\operatorname{tmp}(10 c-1,3)$

denf $=\operatorname{tmp}(100-1,7)$ 


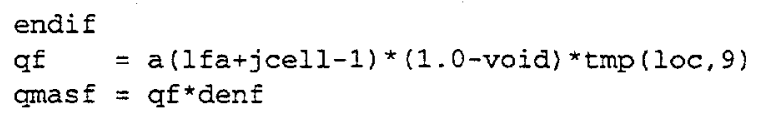

$c$

void $=\operatorname{tmp}(10 \mathrm{c}, 3)$

deng $=\operatorname{tmp}(10 \mathrm{C}, 8)$

if (jcell.gt.jstrt and tmp (loc,10).ge.0.0) then

void $=\operatorname{tmp}(10 c-1,3)$

deng $=\operatorname{tmp}(10 c-1,8)$

endif

gg $=a(1 f a+j c e l l-1) * v o i d * \operatorname{tmp}(10 c, 10)$

gmasg $=q g{ }^{\star}$ deng

c

write (iunit, 2666) ipt,

tsec, conv_p*tmp $(10 c, 1)$

$\operatorname{tmp}(100,5)-c o n v_{-} t$

. $\operatorname{conv} q^{*} q f$

$\operatorname{tmo}(10 c, 4)$ - tmp (10c, 5)

$\operatorname{tmp}(10 c, 7)$

$\operatorname{tmp}(100,1)$

tmp (10c, 3)

gmasg

$\operatorname{tmp}(100,6)-c o n v \_t \quad, \operatorname{tmp}(100,8)$

$\operatorname{tmp}(100,10)$

format (i5.17e15.7)

tmp (10c, 4)-conv_t,

, $\operatorname{tmp}(10 c, 9)$

- gmase

, conv_q* $q g$

, conv_p*tmp $(10 \mathrm{c}, 2)$

$c$

$\operatorname{cc} 2666$ format $(i 5,8 x, f 8.1,7 f 10.2,2 x, f 10.1,2 f 10.4,2 x$, e12.4, f10.4,

cc

$\&$

$3 \mp 10.1, f 10.4)$

endif

endif

endif

enddo

$c$

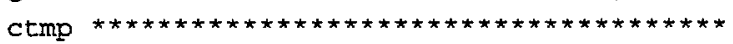

c

130 continue

c

$\operatorname{tmp}(1,1)=a(1 v 1 n+j$ stop $)$

$\operatorname{tmp}(2,1)=a(1 \mathrm{vrn}+j$ stop $)$

if (ioout.eg.1) then

call uncnvt ('vln', tmp, $2,1,-1$ )

endif.

if (getbit (a (1bitn+jstop), 33) ne.0) a (1wfl+jstop) $=-1.111 e-11$

jn=jstop-jstrt +2

write (iout, 140) jn, tmp $(1,1), \operatorname{tmp}(2,1), a(1$ wfl+jstop)

140 format ( $1 \mathrm{x}, 13,84 \mathrm{x}, 1 \mathrm{p}, 2 \mathrm{e} 11.3, \mathrm{e} 10.3$ )

c

c print out heat-transfer solution parameters

c

if (nodes.ne.0) then

write (iout, 200) Iud, luah, Iuha, lupw, lupw, lutp, Iutp

200

format(/6x,'idr liq.htc vap.htc if.htc*a lig.htfx',

1 'vap.htfx temp.chf node-wise wall temperatures'/

2 ' cell' $a, 3 x, a, 2 x, a, 3 x, a, 4 x, a, 6 x, a, 10 x, a)$

$\mathrm{k} 2=\min 0(6+$ nodes, 24$)$

$j n=0$

do $280 \mathrm{n}=1, \mathrm{nn}$

$j 1=j \operatorname{strt}+(n-1) * 10$

$j 2=\operatorname{mino}(j 1+9, j$ stop)

$j 0=j 1-1$

$j 3=j 2-j 0$

do $220 j=j 1, j 2$

$j m I=j-1$ 


\section{$j j=j-j 0$}

$\operatorname{tmp}(j j, 1)=a(1 h i 1+j m 1)$

$\operatorname{tmp}(j j, 2)=a(1 \mathrm{hiv}+j \mathrm{~m} 1)$

$\operatorname{tmp}(j j, 3)=a(1 \operatorname{ch} t i+j m 1)$

$\operatorname{tmp}(j j, 4)=a(1 h i l+j m l) *(a(1 t w n+n o d e s * j m l)-a(1 t l n+j m l))$

$\operatorname{tmp}(j j, 5)=a(\operatorname{lhiv}+j m 1) *(a(1 \mathrm{twn}+$ nodes*jml $)-a(1 \mathrm{tvn}+j m 1))$

$\operatorname{tmp}(j j, 6)=a(1 \operatorname{tch} f+j m 1)$

do $210 \mathrm{k}=7, \mathrm{k} 2$

$\operatorname{tmp}(j j, k)=a(1 \mathrm{t} w n+n o d e s * j m 1+k-7)$

210 continue

220 continue

if (ioout.eq.1) then

call unenvt('hil', tmp $(1,1), j 3,1,-1)$

call uncnve('hiv' , tmp $(1,2), j 3,1,-1)$

call unenvt ('chti', $\operatorname{tmp}(1,3), j 3,1,-1)$

call uncnvt ('qppl', $\operatorname{tmp}(1,4), j 3,1,-1)$

call uncnvt ('qppv', $\operatorname{tmp}(1,5), j 3,1,-1)$

call uncnvt ('tchf', $\operatorname{tmp}(1,6), j 3,1,-1)$

do $230 \mathrm{k}=7, \mathrm{k} 2$

call uncnvt('tw' $\operatorname{tmp}(1, k), j 3,1,-1)$

230 continue

endif

$j 1=j 1-j 0$

$j 2=j 2-j 0$

if $(k 2 . \mathrm{eq} .12)$ then

do $250 j=j 1, j 2$

write(iout, 240) jn+j, a(1iar+j+j0-1), (tmp $(j, k), k=1,12)$

240 format $(1 \mathrm{x}, 13, \mathrm{f5} .1,1 \mathrm{x}, 1 \mathrm{p}, 6 \mathrm{e} 10.3,2 \mathrm{x}, 0 \mathrm{p}, 6 \mathrm{f} 8.2, /(72 \mathrm{x}, 6 \mathrm{f} 8.2))$

250 continue

else

do $270 j=j 1, j 2$

write(iout, 260) jn+j,a(1idr+j+j0-1), (mp $(j, k), k=I, k 2)$

260 format $(1 x, i 3, f 5.1,1 x, 1 p, 6 e 10.3,2 x, 0 p, 6 \pm 8.2, /(72 x, 6 \pm 8.2))$

270 continue

endif

$j n=j n+10$

280 continue

endif

$c$

do $290 \mathrm{j}=j$ strt, jstop

- if $((a(\operatorname{lhla}-1+j)$.ne.0.0) or. $(a(\operatorname{lnva}-1+j)$ ne.0.0)) go to 300

290 continue

go to 330

300 continue

$\operatorname{tmp}(2,1)=0.0$

$\operatorname{tmp}(3,1)=0.0$

do $310 j=j$ strt, jstop

$\operatorname{tmp}(2,1)=\operatorname{tmp}(2,1)+a(1 h l a t w+j-1)-a(1 h l a+j-1) * a(1 t l n+j-1)$

$\operatorname{tmp}(3,1)=\operatorname{tmp}(3,1)+a(1$ hvat $w+j-1)-a(1$ hva $+j-1) * a(1 t v n+j-1)$

310 continue

$\operatorname{tmp}(1,1)=\operatorname{tmp}(2,1)+\operatorname{trmp}(3,1)$

if (ioout.eq.1) then

call uncnvt ('tpow', tmp $3,1,-1$ )

endif

write (iout, 320) (tmp $(j, 1)$, lupw, $j=1,3)$

c

$\operatorname{tmp}(1,1)=a(1$ tcen $)$

$\operatorname{tmp}(2,1)=a(1$ twan $)$

$\operatorname{tmp}(3,1)=a(1$ twen $)$

xepintg=xpintg

if (ioout.eq.1) then

call uncnvt ('eninl', tmp, 3,1,-1) 
caIl uncnvt ('tpow', xepintg, $1,1,-1$ )

endif

if(iflgw.ne.0.and.nodes.gt.0) write(iout, 325) tmp(1,1), lue,

1 xepintg, lue, $\operatorname{tmp}(2,1)$, Iue, $\operatorname{tmp}(3,1)$, lue

320 format (/' total power to the component from all heat-transfer',

1 'surfaces is',1p,e14.6,a/' total power to the liquid is',

2 e14.6,a,' and total power to the gas is', e14.6,a)

325 format $/ /, 1 \mathrm{x}$, 'total convective energy transported to the fluid',

' from component wall:',1pe15.7,a,l,1x,

'total energy input into the component wall=',1pel5.7.

a, /,19x, 'inside convective energy error: absolute='

, 1pe13.5, a, 5x, 'effective=', Ipe13.5,a)

330 continue

c

if (ntsprn.ne.0) then

if (idiags.eq.0) then

write (iout, 150) Iur, lud, luid, lutp, Iuha, luha,

1

lumf, lumf, luv, luv, lud

150

format (/5x, 'dis.sol. p.o.sol. fr.new if.drag

temp.ss. '.

'evap.

cond. mEw.liq. mfw.vap.

'velt.liq velt.gas wE.gas'/' cell ratio',

$a, 3 x, a, 3 x, a, 5 x, a, 4 x, a, 1 x, a, 2 x, a, 3 x, a, 5 x, a, 6 x, a, 6 x, a)$

else if (idiags.eq.1) then

C------For IDIAGS.EQ.1 print stabilizer variables arln and arvn,

c-.----instead of liquid and vapor mass flows.

write(iout, 155) lur, lud, luid, Iutp, Iuha, Iuha,

1 Iur, Iur, Iuv, luv, Iud

155 format (/5x, 'dis.sol. p.0.sol. fx.new if.drag temp.ss. '."

1 'evap. cond. velt.lig velt.gas wf.gas. arvn ' cell ratio.',

$3 a, 3 x, a, 3 x, a, 5 x, a, 4 x, a, 1 x, a, 2 x, a, 2 x, a, 4 x, a, 6 x, a, 6 x, a)$

endif

$j n=0$

do $420 \mathrm{n}=1, \mathrm{n}$

$j 1=j \operatorname{strt}+(n-1) * 10$

$j 2=\min 0(j 1+9, j$ stop $)$

$j 0=j 1-1$

$j 3=j 2-j 0$

do $400 j=j 1, j 2$

$j j=j-j 0$

$j m 1=j-1$

if (idiags.eq.0) then

C------IDIAGS.EQ.0

if (isolut.eq.0) then

$\operatorname{tmp}(j j, 1)=0.0$

$\operatorname{tmp}(j j, 2)=0.0$

else

$\operatorname{tmp}(j j, 1)=a(1$ concn $+j m 1)$

$\operatorname{tmp}(j j, 2)=a(1 s n+j m 1)$

endif

$\operatorname{tmp}(j j, 3)=a($ lrhs $+j m 1)$

$\operatorname{tmp}(j j, 4)=a($ Icifn $+j m I)$

$\operatorname{tmp}(j j .5)=a(I t s s n+j m I)$

$\operatorname{tmp}(j j, 6)=a($ lalven $+j m I)$

$\operatorname{tmp}(j j, 7)=a(1 \operatorname{chtin}+j m 1)$

$\operatorname{tmp}(j j, 8)=a(1 \mathrm{rmvm}+j m 1)-a(\operatorname{Irvmf}+j m 1)$

$\operatorname{tmp}(j j, 9)=a(1$ rvmf $+j m I)$

$\operatorname{tmp}(j j, 10)=a(1 v 1 t+j m I)$

$\operatorname{tmp}(j j, 11)=a(1 v v t+j m I)$

$\operatorname{tmp}(j j, 12)=a(1 w f v+j m 1)$

else if (idiags.eq.1) then

C-----IDIAGS.EQ.1 
if (isolut.eq.0) then $\operatorname{tmp}(j j, I)=0.0$

$\operatorname{tmp}(j j, 2)=0.0$

else

$\operatorname{tmp}(j j, 1)=a(1$ concn $+j m 1)$

$\operatorname{tmp}(j j, 2)=a(1 \mathrm{sn}+j \mathrm{~m} 1)$

endif

$\operatorname{tmp}(j j, 3)=a($ lrhs $+j m I)$

$\operatorname{tmp}(j j, 4)=a(I c i f n+j m I)$

$\operatorname{tmp}(j j, 5)=a(1 t s s n+j m 1)$

$\operatorname{tmp}(j j, 6)=a($ lalven+jm. $)$

$\operatorname{tmp}(j j, 7)=a(1$ chtin+jm1)

$\operatorname{tmp}(j j, 8)=a(\operatorname{larln}+j m 1)$

$\operatorname{tmp}(j j, 9)=a($ larvn $+j m 1)$

$\operatorname{tmp}(j j, 10)=a(1 \mathrm{vlt}+j \mathrm{mI})$

$\operatorname{tmp}(j j, 11)=a(1 v v t+j m 1)$

$\operatorname{tmp}(j j, 12)=a(1 w f v+j m 1)$

endif

400

continue

if (ioout.eq.1) then

if (idiags.eq. 0 ) then

C------IDIAGS.EQ.0

call uncnvt('s' , tmp $(1,2), j 3,1,-1)$

call uncnvt ('cifn', tmp $(1,4), j 3,1,-1)$

call uncnvt ('tssn' $\operatorname{tmp}(1,5), j 3,1,-1$ )

call uncnvt ('alven', tmp $(1,6), j 3,1,-1$ )

call uncnvt ('chtin', tmp $(1,7), j 3,1,-1$ )

call uncnvt ('rmvm', tmp $(1,8), j 3,1,-1$ )

call uncnvt ('rvmf' , tmp $(1,9), j 3,1,-1)$

call uncnvt ('vltn' , $\operatorname{tmp}(1,10), j 3,1,-1)$

call uncnvt ('vvtn' , $\operatorname{tmp}(1,11), j 3,1,-1)$

else if (idiags.eq.1) then

C-D-----IDIAGS.EQ.1

call unenvt ('s' , $\operatorname{tmp}(1,2), j 3,1,-1)$

call unenvt ('cifn', tmp $(1,4), j 3,1,-1$ )

call unenvt ('tssn' $\operatorname{tmp}(1,5), j 3,1,-1)$

call uncnvt ('alven', $\operatorname{tmp}(1,6), j 3,1,-1)$

call uncnvt('chtin', tmp $(1,7), j 3,1,-1)$

call uncnvt ('arln', tmp $(1,8), j 3,1,-1)$

call uncnvt ('arvn', tmp $(1,9), j 3,1,-1)$

call uncnvt ('vltn' , $\operatorname{tmp}(1,10), j 3,1,-1)$

call uncnvt ('vvtn' , tmp $(1,11), j 3,1,-1)$ endif

endif

$j 1=j 1-j 0$

$j 2=j 2-j 0$

write (iout, 410) $(j n+j,(\operatorname{tmp}(j, k), k=1,12), j=j 1, j 2)$

410 format ( $1 x, i 3,1 p, 2 e 9.2,0 p, f 6.3,1 p, 6 e 10.3,2 e 11.3, e 10.3)$ $j n=j n+10$

420 continue

$c$

if (idiags.eq. 0) then

C------IDIAGS.EQ.0

$\operatorname{tmp}(1,1)=a(1$ cifn+jstop)

$\operatorname{tmp}(2,1)=a(1$ rmvm+jstop) $-a$ (1rvmf $+j$ stop)

$\operatorname{tmp}(3,1)=a$ (1rvmf + jstop)

$\operatorname{tmp}(4,1)=a$ (IvIt +jstop)

$\operatorname{tmp}(5,1)=a$ (1vvt +jstop)

if (ioout.eq.1) then

call uncnvt('cifn', $\operatorname{tmp}(1,1), 1,1,-1)$

call uncnvt ('rmvm', $\operatorname{tmp}(2,1), 2,1,-1)$

call uncnvt ('vltn', tmp $(4,1), 2,1,-1)$ 
endif

jn=jstop-jstrt +2

write (iout, 430$) j n,(\operatorname{tmp}(j, 1), j=1,5), a(1 w f v+j s t o p)$

430 format $(1 \mathrm{x}, \mathrm{i3}, 24 \mathrm{x}, 1 \mathrm{p}, \mathrm{e} 10.3,30 \mathrm{x}, 2 \mathrm{e} 10.3,2 \mathrm{e} 11.3$, e10.3)

else if (idiags.eq.1) then

C------IDIAGS.EQ.1

$\operatorname{tmp}(1,1)=a(1 \subset i f n+j$ stop $)$

$\operatorname{tmp}(2,1)=a(1 \mathrm{vlt}+j$ stop $)$

$\operatorname{tmp}(3,1)=a$ (Ivvt +jstop)

if (ioout.eq.1) then

call uncnvt ('cifn', tmp $(1,1), 1,1,-1)$

call unenvt('vItn', tmp $(2,1), 2,1,-1)$

endif

jn $=j$ stop-jstrt +2

435

write (iout, 435) jn, (tmp $(j, 1), j=1,3), a$ (Iwfv+jstop)

format (1x, i3, 24x, 1p, e10.3, 50x, 2e11.3, e10.3)

endif

endif

c

return

end

\section{Fortran file wbreak.f:}

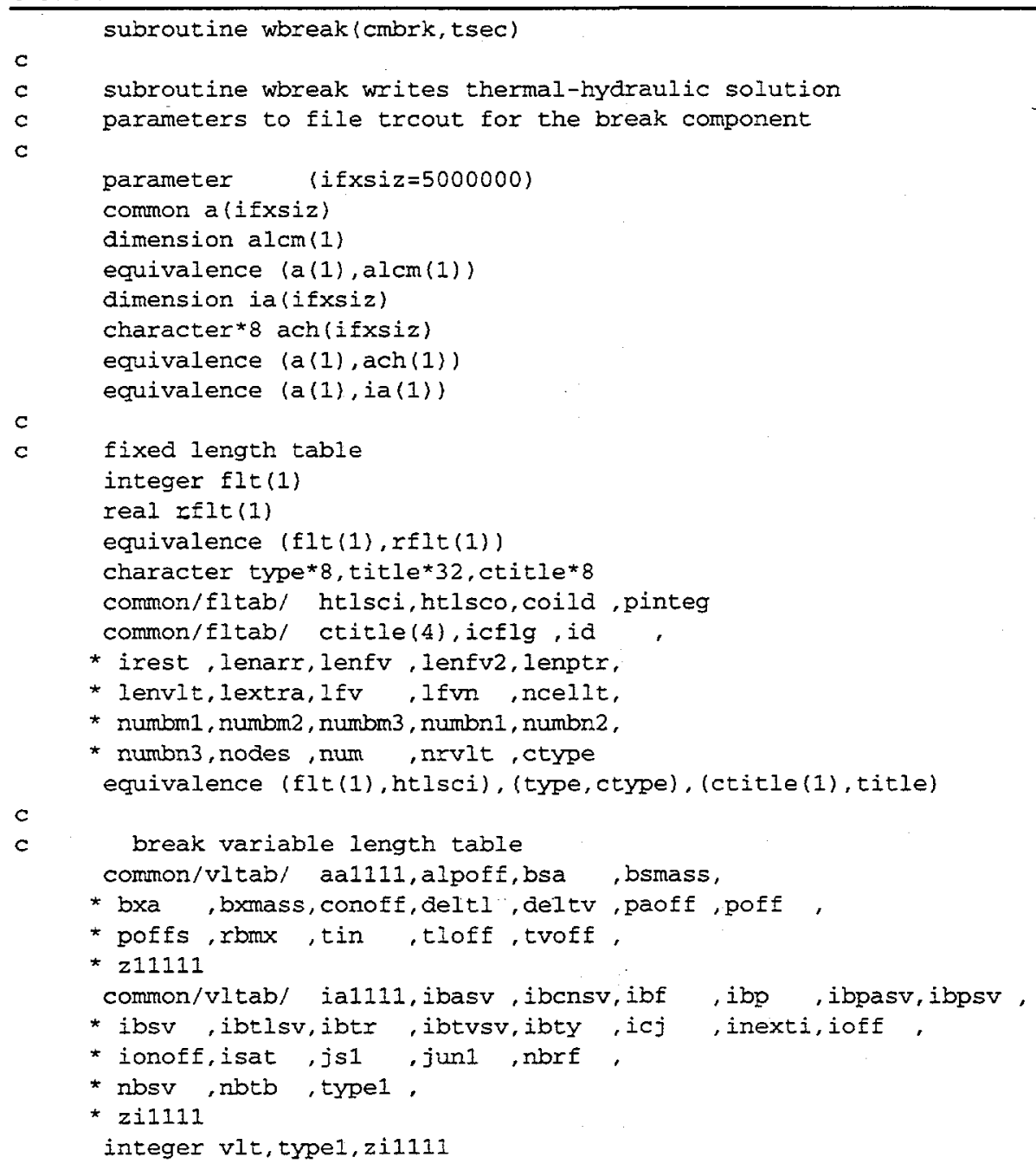


c----comdeck vltdim sets an upper limit to all variable length tables c-----via parameter ivltsz. comdeck genvlt calls vitdim directly, thus c----providing all variable length tables with a uniform length.

c----parameter ivltsz is used to set a uniform length to all

c----variable length tables.

parameter (ivltsz $=270$ )

dimension vit(ivitsz)

equivalence (vit (1), aal111)

c

$c$

c break pointer table

dimension $1 d(3), \operatorname{ldn}(3)$

common/ptab/ lalp, lalpn, lalpa.

* lalpdn, lalv, lalvn, lalve, lalven,

* lara , laran , larel , lareln, larev.

* larevn, larl , larln, larv , larvn.

* Ibit ,lbitn, Ichti, lchtin, Ichtia,

* Ichtan, lcif , Icifn, lcone, Iconen,

* Id , lar , lea , lear , lel

* leln, lev, levn, Igam, Igamn.

* Ihig ,Ihigo, Ihil , Ihilo, Ihiv

* Ihivo , Ip , Ipn , Ipa ,Ipan

* Iqppc, Iqppco, lroa , Iroan , Irol

* Iroln, irov, lrovn, is , Isn

* Itce, Itcen, Itd , Itan, itl

* itir, itv, itva , Itw itwa

* Itwa , Itwan , Itwe , Itwen, Ivi Ivin,

* Ivlt, lvlto. Ivm, lvmn, Ivv

* Ivrn , Ivvt ivvto

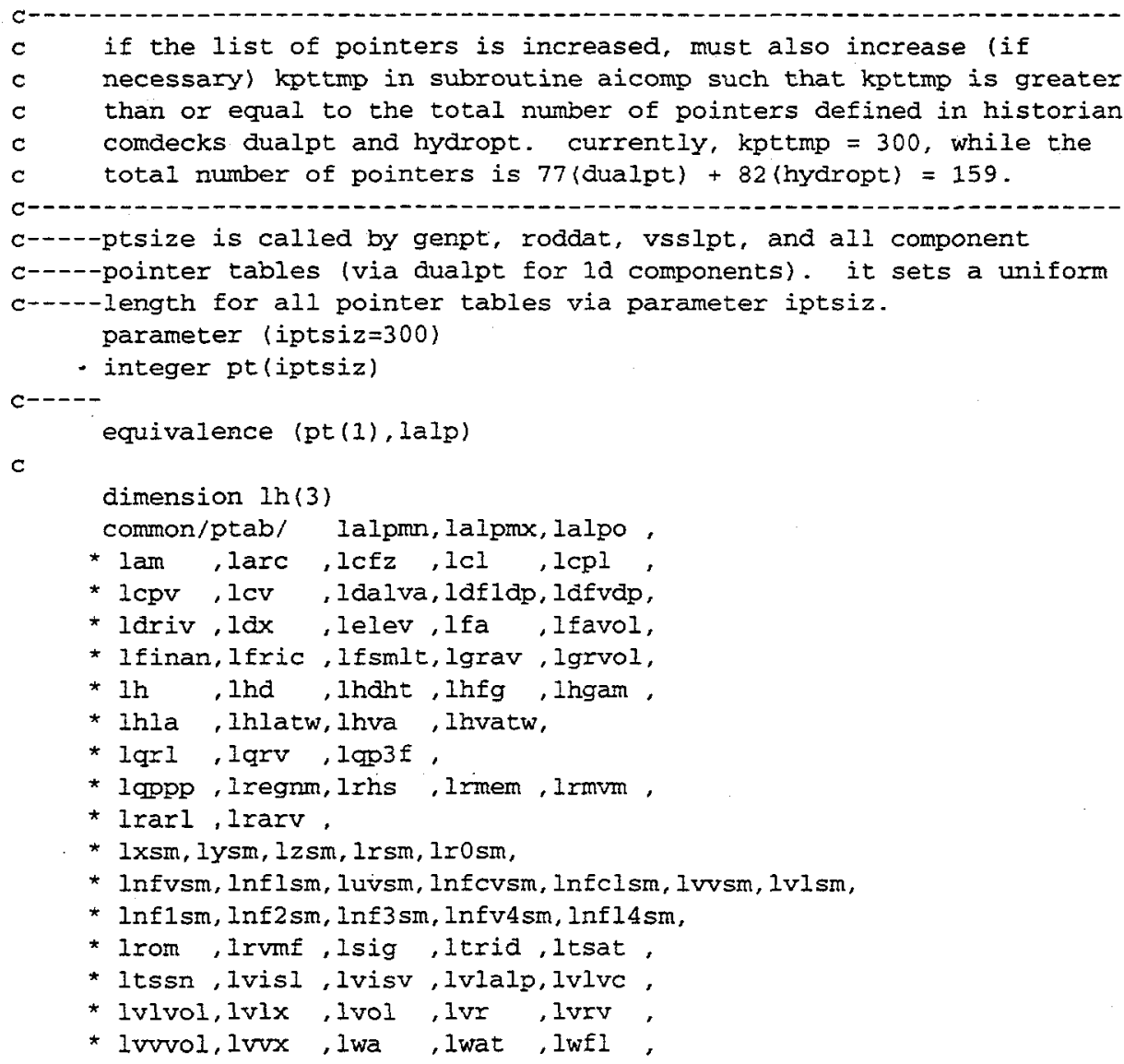


* Iwfv , lwfmfl, lwfmfv

$c$

c if the list of pointers is increased, must also increase (if

c necessary) kpttmp in subroutine aicomp such that kpttmp is greater

c than or equal to the total number of pointers defined in historian

c comdecks dualpt and hydropt. currently, kpttmp $=300$, while the

c total number of pointers is 77 (dualpt) +82 (hydropt) $=159$.

c

c in case new pointers are added after Iwfmfv, then the coding in

c subroutine aicomp (third executable statement) for evaluation of

c the local variable lenptl = locf (lwfmfv) - locf (pt(1)) + 1

c must be changed to use the name of the new pointer at the end

c of this section of the common block / ptab /.

common/ptab/ lalptb, lcontb, 1patb,

* Iptb , Irftb , Itltb, Itvtb

$c$

$c$

common/contrl/ cpuflg, damm, damx , delt,

* deltht, difmin, dprmx , dtlmx , dtmax ,

* atmir , ato , dtrat atrmx ,atsmx ,

* dtvmx , encmax, eps1 ,eps2, epso ,

* ercemx,epss ,etime, htlosi,htloso,

* odelt, powerc,pssmn ,pssmx, rfat,

* rumax , tend , tercmx, timec , timet,

* tmmax , varer, vcms , vcmx , vmaxo,

* vmaxt, vmaxt3, vmcon, vmnew, vmold,

* vmxt3o,xtable, x0sm, y0sm, z0sm,

* omsasm,wsasm, wosasm, tosm (3), xvsm .

* yvsm, zvsm, rmatsm(3,3), $\operatorname{omsm}(3), \operatorname{wsm}(3)$.

* wdsm (3), errsm, dtsm

common/contrl/astep, iadded, iblaus, iccmx ,

* icmp , icmpmx, icp , idiag, idiags, ieccpx,ieos, iff3d,

* ifprep, igeom3, im100, im100x, imfr.

* invan , iofftk, ipak , ipak3d, ipakon, ipkpmp,

* ireset,irsflg, irstfl, isolut, issflg, istdy .

* isttc, ithd , itmin, itpako, jfat, kccmx ,

* lcmptr, levstg, llvflg, ncmn , ncmx ,

* ncontr, nconts, ncontt, norg ,ndial ,nencl,

* newrfd, nfrc1, nfre3, nitav, nitmn.

* nitmx ,nloopp, nosets, nsend, nseo .

* nsmn , nsma , nspl , nspu, ,nsso .

* nstab, nstp , nvgrav, nvoow ,oitmax, sitmax,

* stdyst, transi, motsm, statsm, saxsm

integer dstep, oitmax, sitmax, stdyst,

* transi, statsm, saxsm

c

comnon/units/ ibfadd, ibfadg, ibfadr,

* ibflnd, ibflng, ibflnr, idout, ieeeg, igout,

* ikeybd, imout, in ,inlab ,inproc,

* ioall, iodone, ioerr, iogrf , ioinp,

* iolab, ioout, ioskip, iout ,irstrt,

* itty , iunlab, iunout, lcmcpa, nittab,

* npwtab, nrdy

common/cunits/card

character $* 100$ card

$c$

comon/labelv/factor (150), offset (150)

common/labelv/ih(26), itis (777), itsv(105),

1

ils, ilu, ilun, iold

common/Iabelv/labels (777), Iabsv (2, 105), labun(150), 

1 2 3

character*2 character*3 character* 4 character ${ }^{\star} 5$ character ${ }^{*} 6$ character*7

labels, labun, luid, lur, lusp, Iusz, Iutm character*9 luha character*10 lucp character*11 luph character*12 luhx, lutc, runcb character*13 luh, luncb, lupcb character*14 labsv character*19 ludh character*26 alpbet

c warning - ptrs common block variables

c il1111 to $\mathrm{nzzzzz}$ are zero initialized

c

common/ptrs/ il1111,1bd, lcntl,

* Icompt, lcontp, Icontr, ldra ,ldrc

* licvs, liitno, lijvs, lilcmp, liou, lisvf

* livcon, livijn, ljout, ljseq, ljun ,

* Ilcmhs, 11con , Iloopn, Imatb , Imcmsh,

* Imsct, Inbr , Injn , Insig, Insigp,

* Invenl, lorder, Iprptb, lptbln, ltitle,

* Ivsi, lwo

common/ptrs/ laol, laou, laov,

* lapvc , lapvcv, larel , larev , larl ,

* Idrv, ldvb, lidpcv, lod, 1vrh ,

* jaol, jaov, jarv, jarl, jod,

* jarel, jarev, jara , jarc ,jnjun,

* lilprb, livlfc, livvto, Iivito common/ptrs/ lbvec, lbw , lamat.

* lemat, Ienfxd, Ifxd ,Irmat, Ivmat ,

* Ivssc, Ivssip, nclear, nmat ,nvcell.

* nzzzzz

dimension ipt (76)

equivalence (ipt (1), i11111)

c

C

dimension $\operatorname{tmp}(4)$

c

write(iout, 100) num, junl

100 format $/ /$ ' the component type is a break, component number is'.

1 $c f=0.0$

$c e=0.0$

c

iflgw $=0$

xxpow $=0.0$

itee $=0$

call ecomp(num, 1, I, iflgw, 0, cf, xxpow, ce, tsec, itee)

C

if (( (stdyst.eq.2).or. (stdyst.eq.4)).and. (ia (lcontp).gt.0)) then do $160 \mathrm{i}=1$, ia (1contp)

$j 2=1$ cont $p+20 * i$

$j 1=j 2-17$ 
do $110 j=j 1, j 2$

if (ia $(j)$.eq.num) go to 120

110 continue

go to 160

120 continue

do $130 \mathrm{n}=1$, ncontr

if $(i a(j 1-2)$.eq.ia $(1 \operatorname{cont} r-5+5 * n))$ go to 140

130 continue

go to 160

140 continue

$k=1 \operatorname{cn} t 1+10+6 * i a(I \operatorname{cn} t 1+5)+7 * i a(\operatorname{len} t I+1)$

$1+17 *(i a(1$ cnt $1+2)$-ncontr-ncont $t+n)$

write (iout, 150) a (k), ia(j1-2)

150 format //' the pressure of this break component has been',

1 'adjusted by the factor',1p,e12.5/' by a constrained',

2 ' steady-state controlier applied to component', i4)

160 continue

endif

cmork=bsmass

tmp (1) =bxnass

$\operatorname{tmp}(2)=$ bxa

$\operatorname{tmp}(3)=$ bsmass

$\operatorname{tmp}(4)=$ bsa

if (ioout.eq.1) then

call uncnvt ('bxmass', tmp $(1), 2,1,-1)$

call uncnvt ('bsmass', $\operatorname{tmp}(3), 2,1,-1)$

endif

write(iout, 170) tmp (2), Iumf, tmp (4), lum

170 format (/' ncd-gas mass inflow to the break is', Ip, el3.5,a,

1 ' and total ncd-gas mass in to the break is',e13.5,a)

write(iout, 180) tmp(1), Iumf, tmp (3), lum

180 format (' coolant mass inflow to the break is',1p,e13.5.a,

- and total coolant mass in to the break is', e13.5,a)

c

return

end

\section{Fortran file wcomp.f:}

subroutine wcomp (comptr)

subroutine wcomp writes the control-parameter data to file

trcout and calls the w-subroutines which write out selected

component thermal-hydraulic solution data to file trcout

common block variables used:

comptr $=$ pointer to component data

ifree = first location in a array available for use

lenptr $=$ length of the pointer common block

lentbl $=$ length of the fixed-iength common block

lenvit $=$ length of the variable-length common block

ncomp $=$ number of components

nstep = time-step number

character*8 $\mathrm{nm}(5,11)$

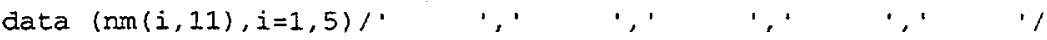

data $(\operatorname{nm}(i, 1), i=1,5), 1,1,11,1,1,1,111$

data $(\operatorname{nm}(i, 2), i=1,5) /\left(22222^{\prime}, 2^{\prime}, ' 22222^{\prime}, ' 2 \quad ', 22222^{\prime} /\right.$

data $(\operatorname{nm}(i, 3), i=1,5) / 33333^{\prime}, \circ \quad 3{ }^{\prime}, 33333^{\prime}, 3^{\prime}, .33333^{\prime} /$

data $(\operatorname{nm}(i, 4), i=1,5) / .4 \quad 4^{\prime},{ }^{\prime} 4 \quad 4^{\prime},{ }^{\prime} 44444^{\prime},{ }^{\prime} \quad 4^{\prime},{ }^{\prime} \quad 4^{\prime} /$

data $(\operatorname{mm}(i, 5), i=1,5) / .55555^{\prime}, .5 \quad$ '. $55555^{\prime}, \cdot \quad 5 \cdot, 55555^{\prime} /$ 
data $(\mathrm{nm}(i, 6), i=1,5) / \cdot 66666^{\prime}, \cdot 6$

data $(\mathrm{nm}(i, 7), i=1,5) / \cdot 77777^{\circ}$,

\begin{tabular}{|c|c|}
\hline ', $66666^{\prime}, \cdot 6$ & $6^{\prime}, \cdot 66666^{\prime}$, \\
\hline$\because 7 \quad \because 7$ & $\cdot, 7$ \\
\hline $8^{\prime}, 88888^{\prime}, \cdot 8$ & $8^{\prime},,^{\prime} 88888^{\prime}$ \\
\hline $9 \cdot, 99999:$, & 9', .99999' \\
\hline $0 ., 000 ., 0$ & $0 ., 00000$ \\
\hline
\end{tabular}

$c$

data $\operatorname{lnm}(i, 8), i=1,5) / .88888^{\prime}, \cdot 8$

data $(\operatorname{nm}(i, 9), i=1,5) / .99999^{\prime}, \cdot 9$

$0 ., \cdot 0$

ctmp

character $* 10$ filename

character $* 80$ commnt

logical first

save first

parameter (maxgpts $=500$ )

common /tecplot/iicomp (maxgpts), i icell (maxgpts), ictype (maxgpts), $\&$ id_leg (maxgpts), npoints, iinl

ctmp

$c$

common/contrl/ cpuflg, dammc, damx, delt,

* deltht, difmin, dprmx , atlmx , dtmax

* dtmin, ato ,atrat ,atrmx , atsmx ,

* dtvmx , encmax, eps1 ,eps 2 ,epso ,

* excemx, epss , etime, htlosi,htloso,

* odelt, powerc, pssmn , pssmx , rfat,

* rvmax , tend , tercmx, timec, timet,

* tmmax , varer, vcmn , vcrnx ,vmaxo,

* vmaxt, vmaxt3, vmcon, vmnew, vmold,

* vmxt3o,xtable, x0sm, yosm, z0sm,

* omsasm, wsasm, wdsasm, t0sm(3), xvsm ,

* yvsm, zvsm, rmatsm $(3,3), \operatorname{omsm}(3), \operatorname{wsm}(3)$.

* wasm(3), errsm, atsm

common/contrl/ dstep, iadded,iblaus, iccmx ,

* icmp, icmpmx, icp , idiag, idiags, ieccpx,ieos, iff3d,

* ifprep, igeom3,im100, imlo0x,imfr,

* invan , iofftk, ipak , ipak3d,ipakon, ipkpmp.

* ireset, irsflg, irstfl, isolut, issflg, istdy.

* isttc, ithd, itmin, itpako,jfat, kccmx .

* Icmptr, levstg, Ilvflg, ncmn , ncmx

* ncontr, nconts, ncontt, ncrg ,ndial, nencl,

* newrfa, nfrcl , nfrc3, nitav, nitmn,

* nitmx ,nloopp, nosets, nsend, nseo ,

* nsmin , nsmx ,nspl, nspu, ,nsso,

* nstab , nstp , nvgrav, nvpow , oitmax, sitmax,

* stdyst, transi, motsm, statsm, saxsm

integer dstep, oitmax, sitmax, stdyst,

* transi, statsm, saxsm

c

common/dimen/ ifree, jnvssl, kvel1t,

* kvel2t, kvel3t, last, laim, lenbd, lendim,

* lentbl, lfree , llast , Imldp , Inlapv, Inrdpt,

* locrdp, Istart, Istrdp, Iver, main

* memflg,moff, ncomp, ncompt,

* nhtstr, njnmx , njnt ,njun ,nloops,

* ninvssl, npx ,nstgj , nthm ,nthmld,

* nthm3d, numtcr, nvcon , nvelx , nvely,

* nvelz, nwrda

$c$

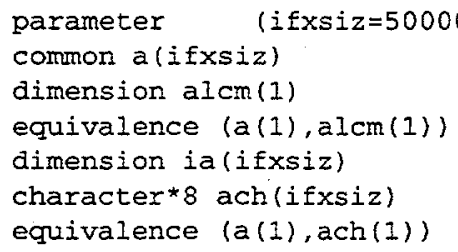




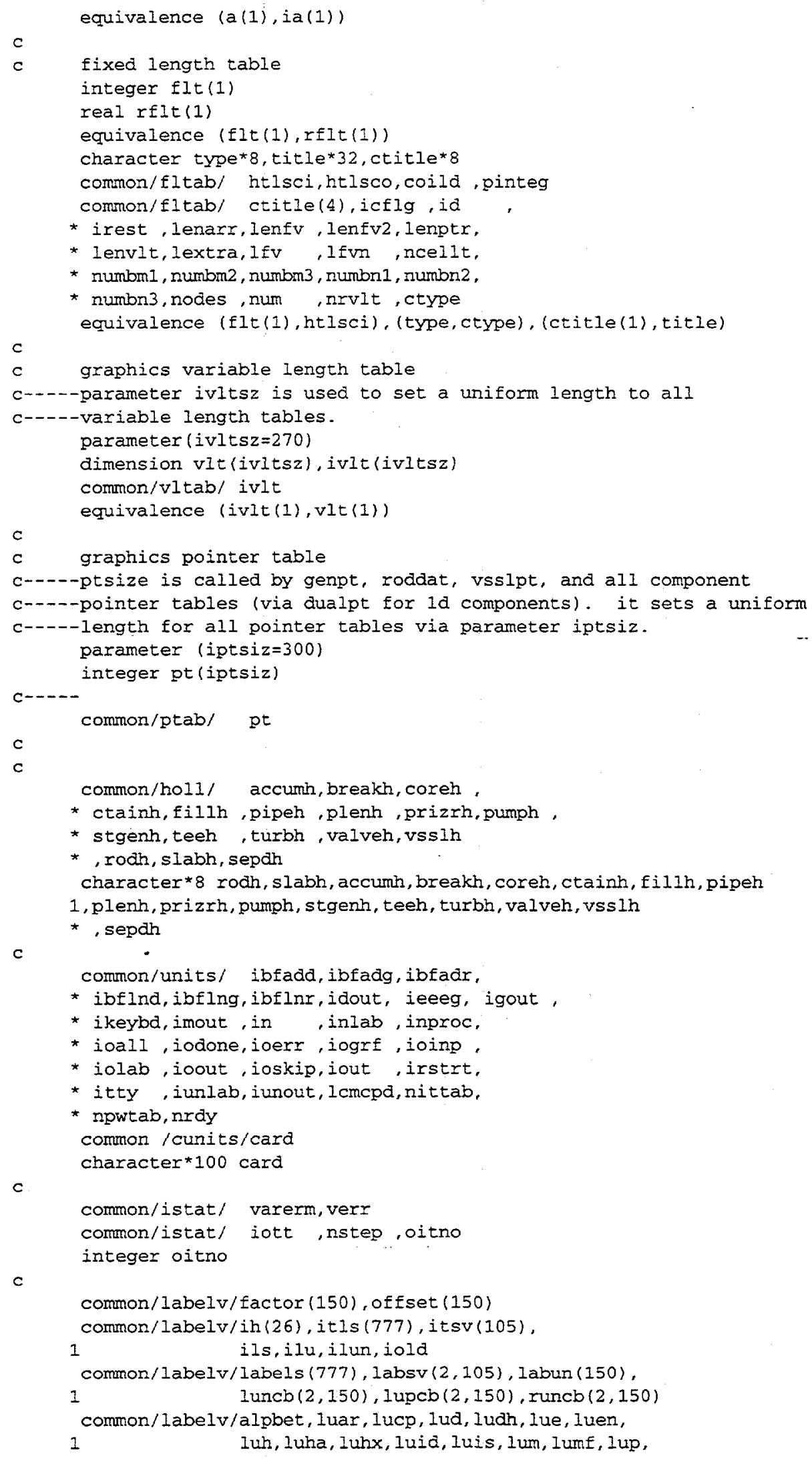


character*2 lud, Iutp, lus

character*3 lut, Iuz

character*4 luar, lue, lum, luvo

character*5 lup, lupd, luv, luvf

character*6 luis

character*7 Iuen, Iumf, lupt, lupw

character* 8 labels, Iabun, Iuid, lur, Iusp, Iusz, Iutm

character*9 luha

character*10 lucp

character*11 Iuph

character*12 luhx, lutc, runcb

character*13 1uh, Iuncb, lupcb

character*14 labsv

character*19 1udh

character*26 alpbet

common/timer/ adate, atime,cput ,

* timcpu, timei , timiom, timsys, timtot

common/timer/ nstept

warning - ptrs common block variables

i11111 to nzzzzz are zero initialized

common/ptrs/ i11111,Ibd ,Icntl.

* lcompt, lcontp, lcontr, Idra , Idrc

* licvs, Iiitno, lijvs, lilcmp, liou , Iisvf,

* livcon, Iivljn,ljout, Ijseq, ljun ,

* Ilcmhs, I Icon, Iloopn, Imatb, Imcmsh,

* Imsct, Inbr , Injn, Insig, Insigp.

* Invcnl, lorder, lprptb, lptbln, ititle.

* Ivsi , Iwp

common/ptrs/ laol, laou , laov

* Idpvc , ldpvcv, ldrel , Idrev , Idrl

* Iarv , lavb, Iidpcv,10a , Ivrh ,

* jaol ,jaov ,jarv ,jarl ,jod

* jarel, jarev, jara , jarc ,jnjun ,

* Iilprb, livlfc, livvto, livlto

common/ptrs/ Ibvec, lbw, lamat,

* Iemat, lenfxa, Ifxa ,Irmat, Ivmat,

* Ivssc , Ivssip, nclear, nmat ,nvcell.

* nzzzzz

dimension ipt (76)

equivalence (ipt (1), i11111)

$c$

common /stncom/ stnmax, tmstnu, tlamin, tmtid

common /stncom/ istnu, jstnu, kstnu, nstnu

common /stncom/ itldm, jtldm, ktldm, ntldm

character*8 lun, status $(2,3)$

character*14 rI(5), sp

equivalence (n1, numbn1), (n2, numbn2), (n3, numbn3)

equivalence (m1, numbm1), (m2, numbm2), (m3, numbm3)

dimension comptr (1), tmp (6)

integer arptr, comptr

data status/' on-r', 'everse ', ' $0^{\prime}$, 'ff

1

on-f', 'orward '/, sp/'

, ', 
c

if (first) then

iinI $=40$

first $=$.false.

c

c Open and read in the selected point where graphical data

$c$ requested.

$c$

open (iinl, file='graphin', status='old')

read (iin1, '(a80)') commnt

read (iin1, *) npoints

c

c If requested number of graphics points greater than maxgpts,

$c$ then write error message and terminate.

$c$

if (npoints.gt.maxgpts) then

write $(*, 2090)$ maxgpts

write (iout, 2090) maxgpts

2090

format $(/)$,

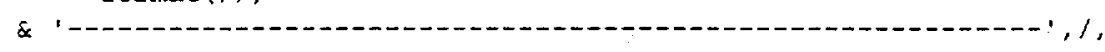

$\& \cdot * \star *$ ERROR $* * * 1,1$,

$\&$ ' No. of graphics points requested in graphin file exceeds', /,

\& ' maximum allowed $(1, i 3,1)$. Program terminated.', /,

\& $\cdot * \star *$ ERROR $* * * 1,1$,

$\&$

stop

endif

$\mathrm{c}$

read (iinl, '(a80)') commnt

do ipts $=1$, npoints

read (iinl,*) iicomp(ipts), iicell(ipts), ictype(ipts),

$\&$

endào

id_leg (ipts)

$c$

c Open summary tecplot graphics file (one file will contain all

c component graphics; external routine "tecsplit" is requred

c to split out the graphics into multiple tecplot files for

c each requested graphics point):

$c$

write $(*, 2100)$

write (iout, 2100)

2100

format $(/)$,

\& ' '-

\& " Tecplot graphics sumary file generated: "tecsum.grf" ',/,

\& ' - '

iunit $=$ iinl

filename $=$ 'tecsum.grf'

open (iunit, file=filename, status=' unknown')

write (iin1, 2105)

2105 format

\& 'This file contains graphics input from TRAC for tecsplit')

c

c Adjust cell id up one when component is a tee to be consistent

$c$ with storage scheme used in TRAC when cell is in the second

$c$ leg of that tee.

c

do ipts=1, npoints

if (ictype (ipts) .eq.2 .and. id_leg(ipts).eq.2) then

iicell(ipts) $=i$ icell (ipts) $+I$

endif

endio

c 
endif

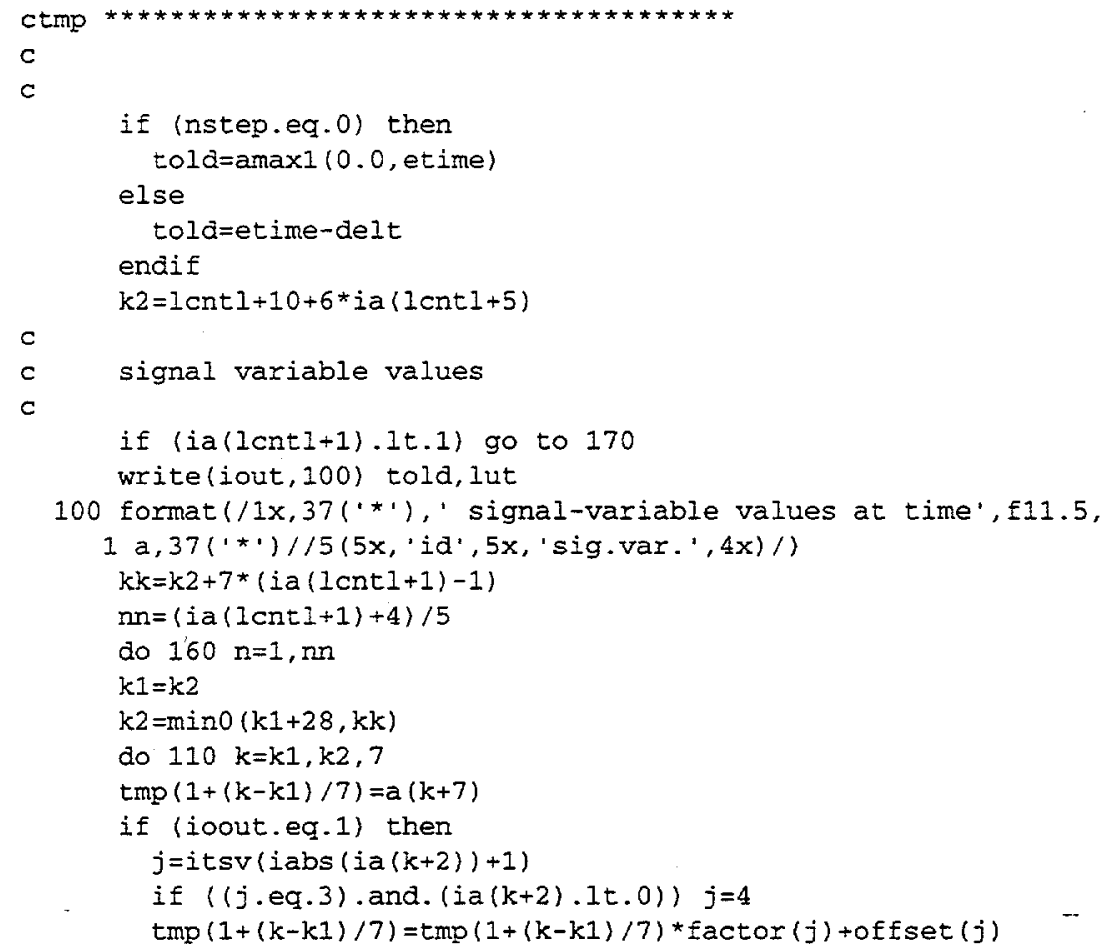


$\mathrm{k} 2=\min 0(\mathrm{k} 1+68, \mathrm{kk})$

do $190 \mathrm{k}=\mathrm{k} 1, \mathrm{k} 2,17$

$\operatorname{tmp}(1+(k-k 1) / 17)=a(k+17)$

if ((ioout.eq.1).or. (iunout.eq.1)) then

lun=ach $(11+2)$

call uncnvt (Iun, tmp $(1+(k-k 1) / 17), 1,1,-i o o u t)$

$\mathrm{rl}(1+(\mathrm{k}-\mathrm{k} 1) / 17)=\mathrm{sp}(1: 2) / /$ runcb $($ ioout +1 , it $1 \mathrm{~s}($ iold $))$

endif

$11=11+6$

190 continue

write (iout, 120) (ia $(k+1), \operatorname{tmp}(1+(k-k 1) / 17), k=k 1, k 2,17)$

if (iunout.eq.1) then

write (iout, 150) $(\mathrm{r} I(1+(\mathrm{k}-\mathrm{k} 1) / 17), \mathrm{k}=\mathrm{k} 1, \mathrm{k} 2,17)$

endif

$k 2=k 2+17$

200 continue

$k 2=11$

c

c

$\mathrm{c}$

210 continue

if $($ ia $(1 \operatorname{cnt} 1+4) .1 t .1)$ go to 270

$11=k 2$

$k 2=k 2+i a(1 \operatorname{cn} t I+4)+5 * i a(1 \ln t I+6)$

write (iout, 220) told, lut

220 format $\left(/ 1 x, 41\left({ }^{\prime} *\right), \cdot\right.$ trip set status at time', f11.5,a,

$140\left({ }^{*} \cdot\right) / / 5(5 x$, 'id', $4 x$, 'set status', 3x)/)

$\mathrm{k} 1=\mathrm{k} 2$

$\mathrm{k} 2=\mathrm{k} 1+80 *(\mathrm{ia}(1 \operatorname{cnt} 1+4)-1)$

write (iout, 230) (ia $(k+1),($ status $(i, i a(k+3)+2), i=1,2), k=k 1, k 2,80)$

230 format $(i 7,2 a, i 8,2 a, i 8,2 a, i 8,2 a, i 8,2 a)$

c

c

$c$

trip signal values

write(iout, 240) told, Iut

240 format (/1x,39('*'),' trip signal values at time',fll.5,a,

$139\left({ }^{\prime} * 1\right) / / 5\left(5 x,{ }^{\prime} i d^{\prime}, 5 x,{ }^{\prime}\right.$ txp.sig.', $\left.\left.4 x\right) /\right)$

$\mathrm{kk}=\mathrm{k} 2$

$k 2=k 1$

$\mathrm{nn}=(\mathrm{ia}(1 \mathrm{cnt} l+4)+4) / 5$

do $260 . \mathrm{n}=1, \mathrm{mn}$

$\mathrm{k} 1=\mathrm{k} 2$

$\mathrm{k} 2=\operatorname{mino}(\mathrm{kl}+320, \mathrm{kk})$

do $250 \quad k=k 1, k 2,80$

$\operatorname{tmp}(1+(k-k 1) / 80)=a(k+19)$

if ((ioout.eq.1).or. (iunout.eq.1)) then

$1 \mathrm{un}=\operatorname{ach}(1 I+1)$

cal1 uncnvt (lun, tmp $(1+(k-k 1) / 80), 1,1,-$ ioout)

$r l(1+(k-k 1) / 80)=\operatorname{sp}(1: 2) / /$ runcb $($ ioout +1 , itls (iold) $)$

endif

$11=11+1$

250 continue

write (iout, 120) (ia $(k+1), \operatorname{tmp}(1+(k-k 1) / 80), k=k 1, k 2,80)$

if (iunout.eq. 1 ) then

write (iout, 150) $(r 1(1+(k-k 1) / 80), k=k 1, k 2,80)$

endif

$k 2=k 2+80$

260 continue

$c$

270 continue

if (stnmax.ne.0.0) then

if (istnu.eq. 0 ) then 


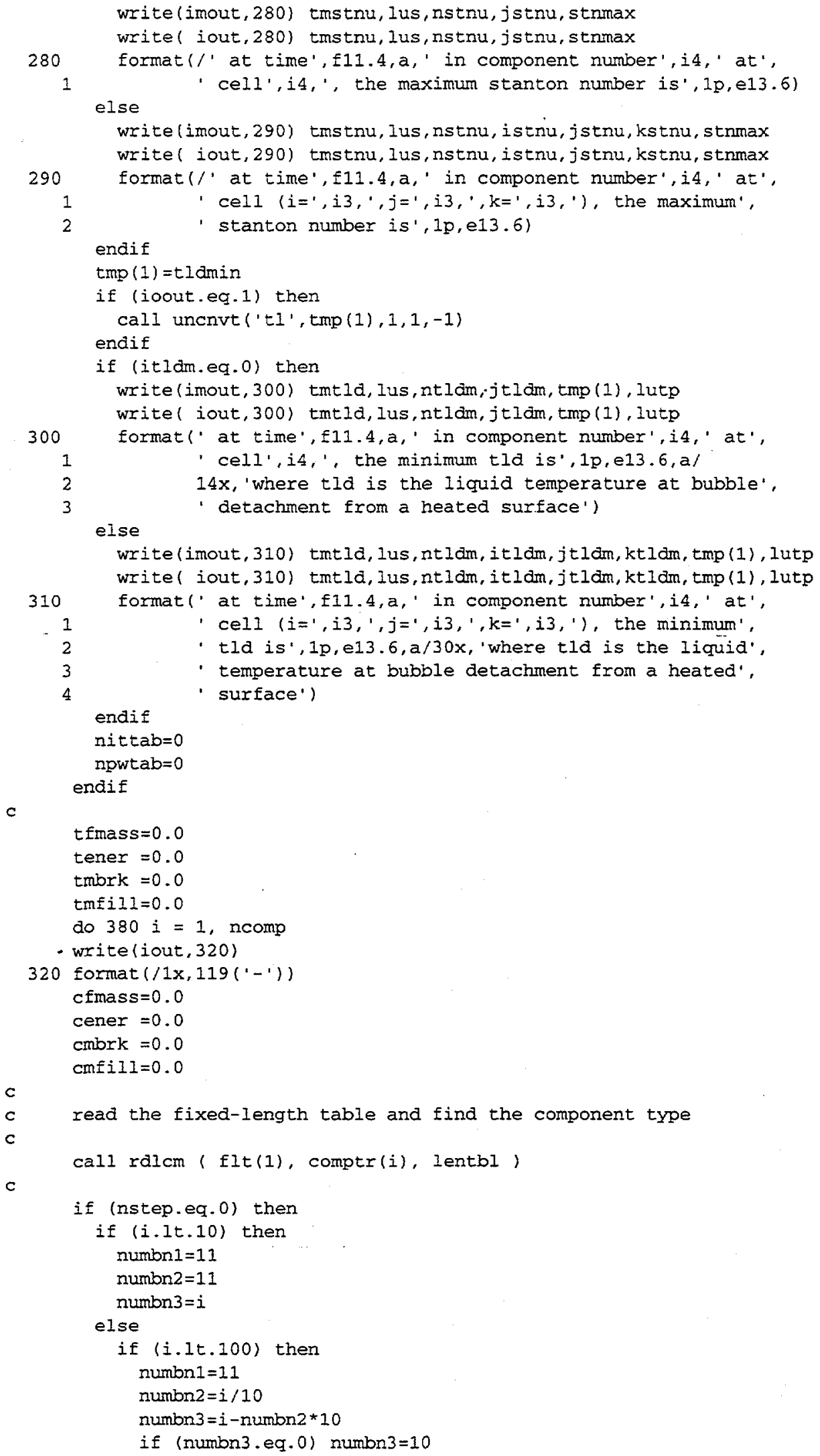




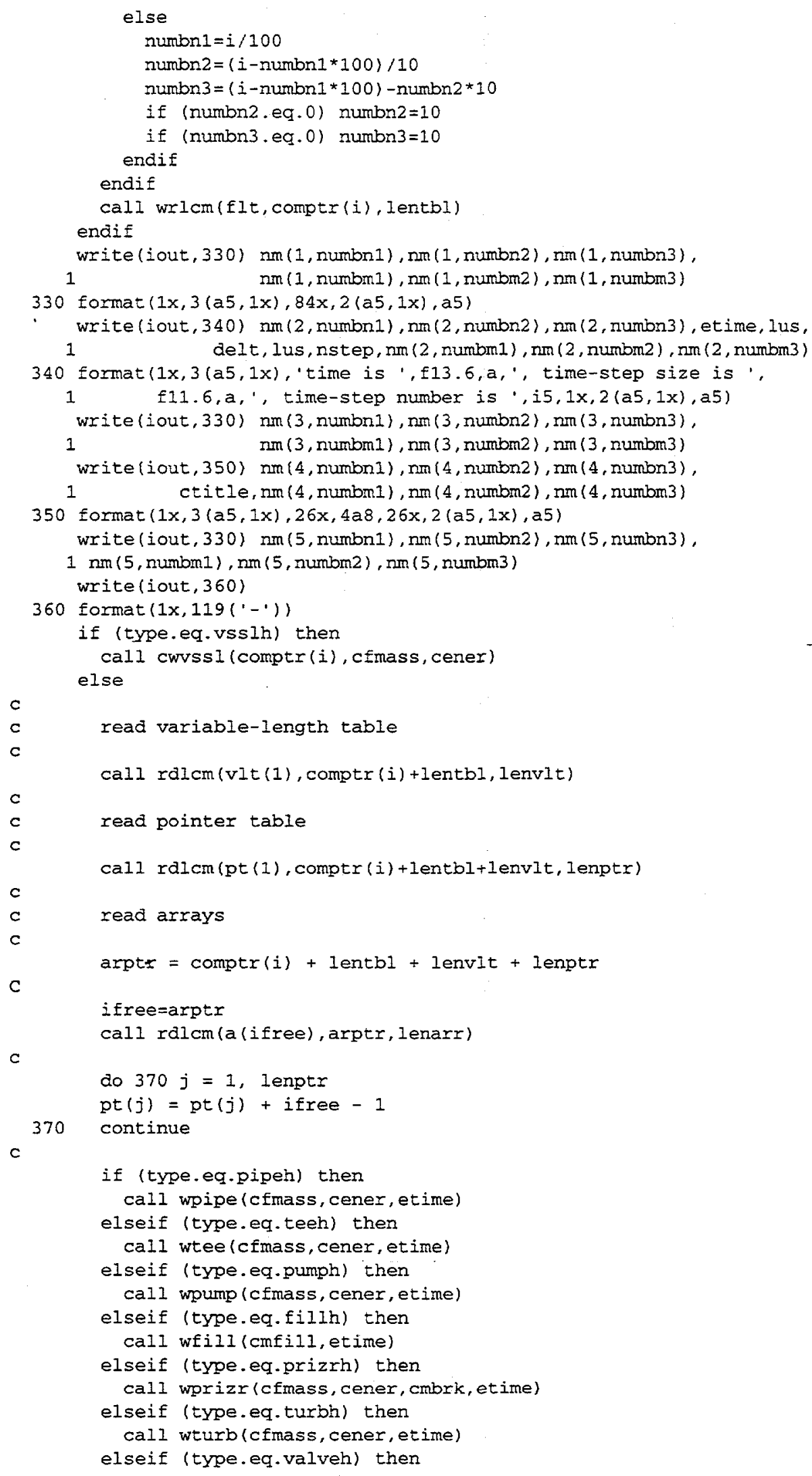


call wvlve (cfmass, cener, etime)

elseif (type.eq.sepdh) then

call wtee (cfmass, cener, etime)

elseif (type.eq.breakh) then

call wbreak (cmbrk, etime)

elseif (type.eq.plenh) then

call wplen (cfmass, cener, a (lbd), lenbd, etime) endif

endif

tfmass $=$ tfmass + cfmass

tener $=$ tener+cener

tmbrk $=$ tmbrk +cmbrk

$\operatorname{tmfill=tmfill\div \mathrm {cmfil}}$

380 continue

if (nhtstr.gt.0) call whtstr

write (iout, 390)

390 format (//' system results')

$t \operatorname{tmp}(1)=t$ fmass

$\operatorname{tmp}(2)=$ tmbrk

$\operatorname{tmp}(3)=\operatorname{tmfil}$

$\operatorname{tmp}(4)=$ tener

$i=1$

if (nstep.gt.0) then

$i=3$

$\operatorname{tmp}(5)=h t l o s i$

$\operatorname{tmp}(6)=h t$ loso

endif

if (ioout.eq.1) then

call uncnvt ('tfmass', $\operatorname{tmp}(1), 3,1,-1$ )

call uncnvt('tener', $\operatorname{tmp}(4), i, 1,-1)$

endif

if (nstep.gt.0) then

write (iout, 400) tmp (5), Iue, tmp (6), lue

400 format $/ /$ total power loss by $1-\mathrm{d}$ comp.wall is',1p, el4.6, a,

1 ' on the inner surface and',e14.6,a,' on the outer',

2

' surface'

endif

write (iout, 410) tmp (4), lue, tmp (1), lum

410 format (/23x,' system total coolant energy is',1p,e14.6,a//

$125 \mathrm{x}$, 'system total coolant mass is', e14.6, a)

write(iout, 420) tmp(2), Ium

420 format (/' total coolant mass discharged by break components is',

1 Ip,e14,6,a)

write(iout, 430) tmp (3), lum

430 format $(/ 4 \mathrm{x}$, 'total coolant mass injected by fill components is'.

$1 \quad 1 p, e 14.6, a)$

tmtot $=\operatorname{tmp}(1)+\operatorname{tmp}\{2\}-\operatorname{tmp}(3)$

write (iout, 440) tmtot, Ium

440 format (/8x, 'system computed initial total coolant mass is',

$1 \quad 1 \mathrm{p}, \mathrm{el} 4.6, \mathrm{a} / \mathrm{l}$

c

return

end

\section{Fortran file wfill.f:}

subroutine wfill (cmfill, tsec)

subroutine wfill writes thermal-hydraulic solution

parameters to file trcout for the fill component 


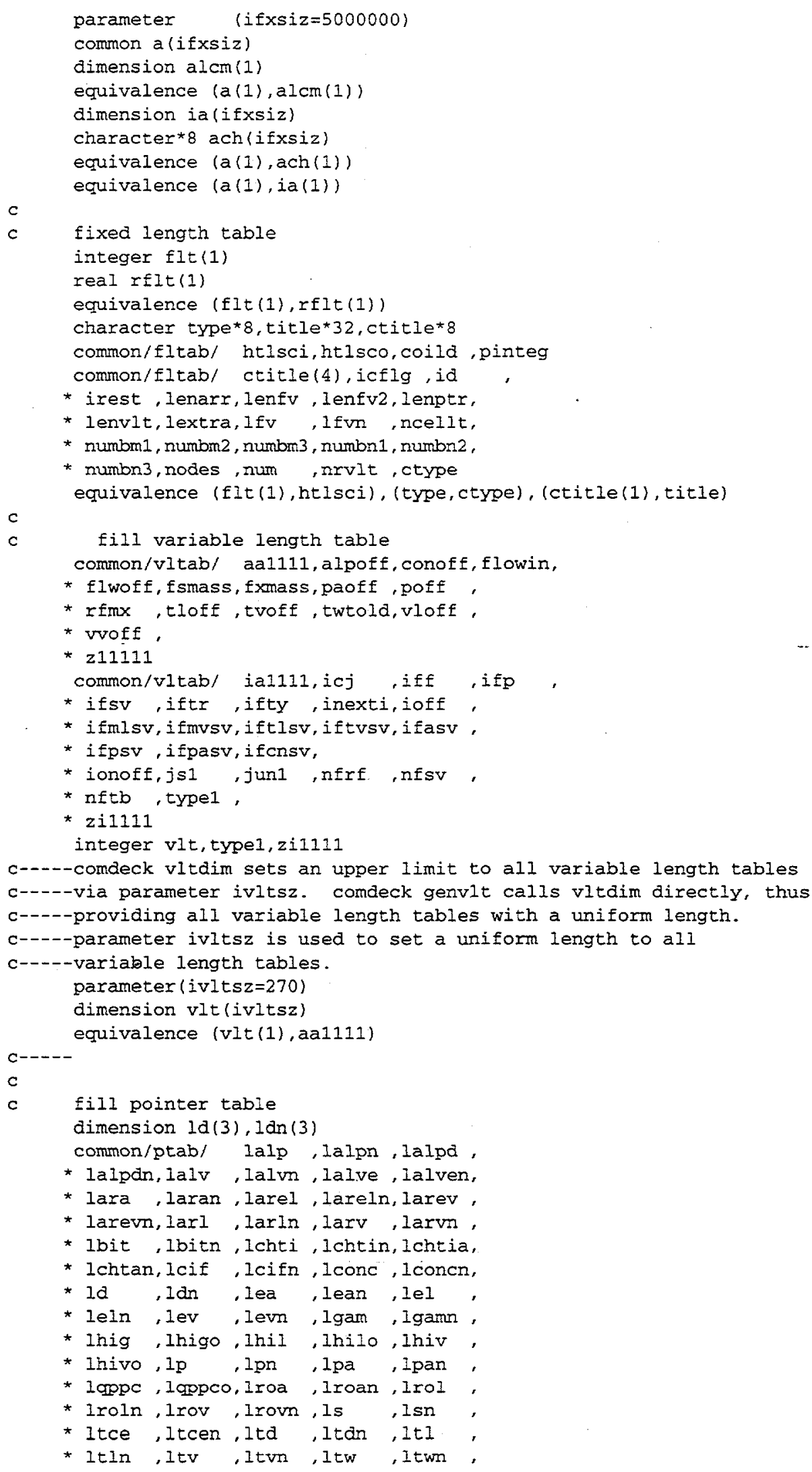




\section{TRAC CODEMODIFICATIONS}

Page:

* Itwa , Itwan, Itwe , Itwen, Ivi , Ivln

* lvit, ivito, Ivm, lvmn, ivv.

* Ivvn, ivvt, 1vvto

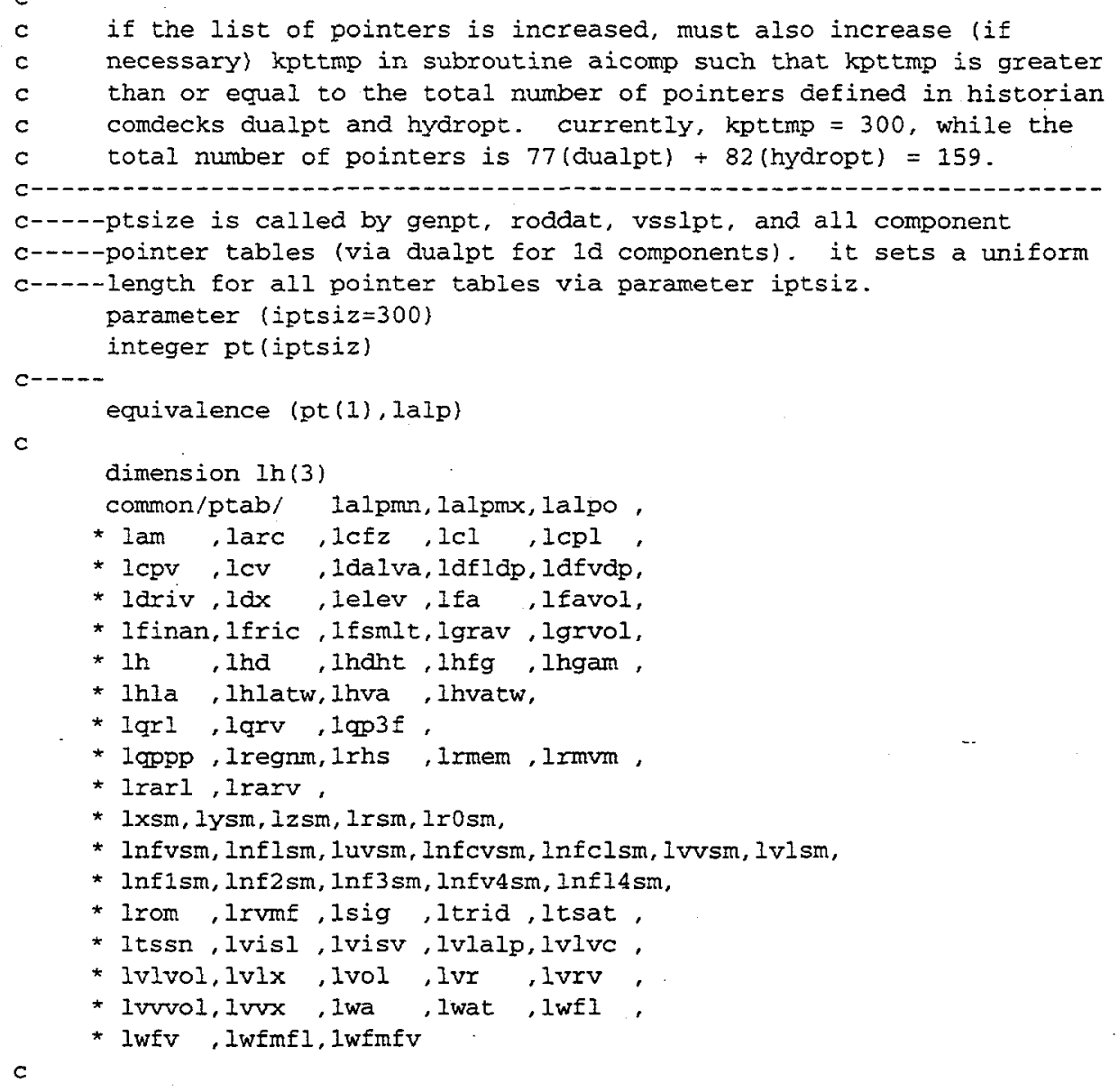

c if the list of pointers is increased, must also increase (if

c - necessary) kpttmp in subroutine aicomp such that kpttmp is greater c than or equal to the total number of pointers defined in historian c comdecks dualpt and hydropt. currently, kpttmp $=300$, while the c total number of pointers is 77 (dualpt) +82 (hydropt) $=159$.

c in case new pointers are added after lwfmfv, then the coding in c subroutine aicomp (third executable statement) for evaluation of c the local variable lenpt1 = locf (Iwfmfv) - locf $(p t(1))+1$

c must be changed to use the name of the new pointer at the end c of this section of the common block / ptab /.

c -...-.-.-

common/ptab/ lalptb, lcontb, lpatb ,

* 1ptb , Irftb, Itltb , Itvtb , Ivmtb.

* Ivvtb

c

$c$

common/units/ ibfadd, ibfadg, ibfadr,

* ibflnd, ibflng, ibflnr, idout, ieeeg, igout,

* ikeyba, imout, in ,inlab, inproc,

* ioall, iodone, ioerr, iogrf , ioinp ,

* iolab, ioout, ioskip,iout ,irstrt,

* itty , iunlab, iunout, Icmcpd, nittab, 
* npwtab, nray

common /cunits/card

character $* 100$ card

c

common/labelv/factor (150), offset (150)

common/labelv/ih(26), itls (777), itsv(105),

1

ils, ilu, ilun, iold

common/labelv/labels (777), labsv (2, 105), labun (150) luncb $(2,150), \operatorname{lupcb}(2,150), \operatorname{runcb}(2,150)$

common/labelv/alpbet, luar, lucp, lud, Iudh, Iue, luen,

3 luh, luha, Iuhx, Iuid, luis, Ium, Iumf, Iup, lupd, Iuph, Iupt, Iupw, Iur, Ius, Iusp, Iusz,

character*2 lud, lutp, lus

character*3 lut, luz

character*4 luar, lue, lum, luvo

character*5 lup, Iupd, Iuv, Iuvf

character*6 luis

character $* 7$ luen, lumf, lupt, lupw

character*8 labels, Iabun, luid, lur, Iusp, Iusz, Iutm

character*9 luha

character $* 10$ lucp

character*11 Iuph

character*12 Iuhx, Iutc, runcb

character*13 Iuh, luncb, Iupcb

character*14 labsv

character*19 ludh

character $* 26$ alpbet

c

dimension tmp (2)

c

write (iout, 100) num, jun1

100 format $("$ the component type is a fill, component number is',

1

$c f=0.0$

$\mathrm{ce}=0.0$

$c$

if $l g w=0$

xxpow $=0.0$

itee $=0$

$c$

call ecomp (num, 1, I, iflgw, 0,cf, xxpow, ce, tsec, itee)

cmfill=fsmass

$\operatorname{tmp}(1)=$ fxmass

$\operatorname{tmp}(2)=$ fsmass

if (ioout.eq.1) then

cal1 uncnvt ('fxmass', $\operatorname{trp}(1), 1,1,-1$ )

call uncnvt ('fsmass', $\operatorname{tmp}(2), 1,1,-1)$

endif

write(iout, 110) tmp (1), lumf, tmp (2), Ium

110 format (/" fill coolant mass outflow is',1p, el3.5,a,

- and total mass from the fill is',el3.5,a)

c

return

end

\section{Fortran file whtstr.f:}

subroutine whtstr

$c$

subroutine whtstr writes writes thermal-hydraulic solution

parameters to file trcout for the heat-structure component 
integer $f I t(1)$

real rflt (1)

equivalence (flt(1), rflt(1))

character type*8,title*32, ctitle*8

common/fltab/ htlsci,htlsco, coild, pinteg

common/fltab/ ctitle(4),icflg, id

* irest, lenarr, lenfv, lenfv2, lenptr,

* lenvit, lextra, lfv , Ifvn, ncellt,

* numbm1, numbm2, numbm3, numbn1, numbn2,

* numbn3, nodes, num ,nrvit, ctype equivalence (Elt (1), htIsci), (type, ctype), (ctitle(1), title)

c

common/units/ ibfadd, ibfadg, ibfadr,

* ibflnd, ibflng, ibflnr, idout, ieeeg, igout ,

* ikeybd, imout, in ,inlab, inproc,

* ioall, iodone,ioerr , iogrf , ioinp.

* iolab, ioout, ioskip, iout ,irstrt,

* itty ,iunlab, iunout, lcmcpd, nittab,

* npwtab,nrdy

common /cunits/card

character $* 100$ card

$c$

common/istat/ vaxerm, verr

common/istat/ iott, nstep, oitno

integer oitno

c

common/labelv/factor (150), offset (150)

common/labelv/ih (26), itls (777), itsv(105),

1 ils, ilu, ilun, iold

common/labelv/labels (777), labsv (2, 105), Iabun (150),

1 luncb $(2,150), 1$ upcb $(2,150)$, runcb $(2,150)$

common/labelv/alpbet, luar, lucp, lud, ludh, Iue, luen,

1

1
2

3 Iun, Iuha, Iuhx, luid, Iuis, Ium, Iumf, Iup, lupd, Iuph, Iupt, Iupw, Iur, Ius, Iusp, Iusz, lut, Iutc, Iutm, lutp, luv, luvf, Iuvo, Iuz

character*2 lud, Iutp, Ius

character*3 lut, Iuz

character*4 luar, lue, 1um, luvo

character*5 Iup, lupd, Iuv, luvf

character*6 luis

character*7 luen, lumf, lupt, lupw

character ${ }^{*}$ labels, Iabun, luid, lur, Iusp, Iusz, Iutm

character*9 luha

character*10 lucp

character*11 luph

character*12 luhx, 1utc, runcb

character*13 luh, Iuncb, 1upcb

character $* 14$ labsv

character*19 Iudh

character ${ }^{2} 6$ alpbet

c warning - ptrs common block variables

c il1111 to nzzzzz are zero initialized

c.

c

ctmp

c

common /tecplot/iicomp (500), iicell (500), ictype (500),

$\&$

id_leg (500), npoints, iin1

dimension tsurfi $(50)$, tsurfo(50)

dimension qsgi (50), qsgo $(50)$, qsfi (50), qsfo(50)

dimension filmfi(50), filmfo(50), filmgi (50), filmgo (50) 


\section{TRAC CODE MODIFICATIONS}

Page:

dimension $t f i(50), t f o(50), \operatorname{tg} i(50), \operatorname{tgo}(50)$

$c$

ctmp

$c$

c

common/ptrs/ il1111,lbd ,lentl,

* 1compt, lcontp, Icontr, Idra , Idrc

* licvs, liitno, lijvs, lilcmp,liou , Iisvf .

* livcon, livljn, ljout, ljseq , ljun

* Ilcmhs, llcon, Iloopn, Imatb, Imemsh,

* Imsct, Inbr , Injn , Insig , Insigp.

* Invenl, lorder, lprptb, lptbln, 1title.

* Ivsi . Iwp

common/ptrs/ laol, laou, laov

* Idpve , Idpvev, Idrel , Idrev, Idrl

* larv , lavb, Iiapcv, lod , Ivrh.

* jaol, jaov, jdrv, jdrl, jod

* jarel ,jarev, jara ,jarc ,jnjun.

* lilprb, livlfc, Iivvto, livlto

common/ptrs/ lbvec, lbw , ldmat,

* Iemat, lenfxd, Ifxd , Irmat , Ivmat .

* Ivssc , Ivssip, nclear, nmat , nvceli,

* nzzzzz

dimension ipt (76)

equivalence (ipt (1), i11111)

$c$

rod data

dimension dtnht (2), atxht (2), sa(2), tk(3),

* ibu(4), irc (4), ircjem (4), ircjtb $(4,4)$

common/vltab/ aal111, amh2, bcro

* bcr1 beff, bppo ,bpp1 ,arfb

* ari ,drio, dtnht, dtpk , dexht ,

* dznht, eneff, extsou, fsi ,fso ,ftci

* ftcm ,ftco ,fucrac,hori ,haro ,hgapo,

* hIi ,hlo , hvi , hvo ,parat.

* plar ,powexp, qratot, reac , reacn.

* react, rmck , rmckn, rpower, rpowpf, rpowr ,

* rpowri, rpowrn, rpowro, rpowto, rpwoff,

-* rpwscl, rrpwmx, rzpwinx, sa , saf

* sat ,shelv, shtd, stimet, tk

* tii, tlo, tneut, tpowi, tpowo.

* tramax, trhmax, tvi , tvo ,watlev,

* width , zpwin , zpwoff, zuptop, zupbot,

* ziptop, zipbot, z11111

common/vltab/ ial1ll, iaf, iaxcnd,ibu .

* idbci , idbco, iext, ionoff, ipatch,

* ipwdep, ipwrad, irc ,ircjfm, ircjtb, irf .

* irftr, irftr2, ixp ,irpwsv, ixpwtr,

* irpwty, isnotb, ittcs, izf , izp , izpwsv,

* izpwtr, Ienrd, Ifvnr, Ifvnr1,1fvr ,

* Ifvr1 , Iiqlev, lndrd, Infvr , Infvr1.

* Inptrr, locrod,mla , mlat , ncrx , nerz ,ndg

* ndgx , ndh , ndhx ,nfbpwt, nfci .

* nfcil ,nfuel, nhist, nint ,nmwrx ,

* nonoff, nopowr, nramax, nrfd ,nrhmax,

* nridr , nrods , nrpwi ,nrpwr , nrpwrf,

* nrpwsv, nrpwtb, nrts , nset ,nset2.

* nzmax ,nzpwrf, nzpwsv, nzpwtb, nzpwi .

* nzpwz, nzznhc, zi1111

integer zi1111 
c----comdeck vitdim sets an upper limit to all variable length tables c-----via parameter ivitsz. comaeck genvlt calis vitdim directly, thus c----providing all variable length tables with a uniform length.

c----parameter ivltsz is used to set a uniform length to all

c-----variable length tables.

parameter (ivltsz $=270$ )

dimension vlt(ivltsz)

equivalence (vit(1), aa1111)

c-----

$c$

c general rod-data pointers

c

common/ptab/ Ibeta, Icdg, Icdgn,

* lcah , lcdhn, Iclen, iclenn, lcpowr,

* ledh , Ifpuo2, Ifta , Igmix , Igmles,

* Igravr, Ihceli, Ihcelo, Ihcomi, Ihcomo,

* Ihigh, Ihs , Ihtmli, Ihtmlo, Ihtmvi,

* 1htmvo, lidrod, 1 lamda, 1 lamdh,

* 11chci, Ilchco, Imatrd, Infax , Inrdx ,

* Intsxx, lpgapt, lplvol, lpowli, lpowlo,

* 1powvi, lpowvo, Ipslen, l radrd, Ircal.

* Ircbm, Ircn , Irctc, Irctf , Irdpwr,

* Iraz , lrpkf , Irpwrf, Irpwrt, lrpwtb,

* Irs, Isrp, itc, Ixn, Ixo,

* 1zpw , lzpwf , 1zpwfb, 1zpwrf, 1zpwtb,

* lzpwzt,lzs , Izzrd

c

c rod-data pointers

c

common/ptab/ lalpr, lalvr, lbitr,

* Ibitrn, Iburn, Icepwn, Icepwo,

* lchtir,lclr , lcnd

* lcnar, lconcr, lcpdr, Icplr, Icpnd .

* lcpvr, lcvr , Idrlat, ldrvat, larz

* ldrzn, lear, Ielr, Iemis, levr,

* Ifinar, lhdr, Ihfgr, Ihganr, Ihgap.

* Ihlar, Ihlatr,Ihlsr , Ihrfg , Ihrfgo.

* Ihrfl, Ihrflo, Ihrfv ,Ihrfvo, Ihrlg,

* Ihrlgo,Ihrli, Ihrllo, Ihrlv, Ihrlvo, Ihqrad, Ihqrdo,

* Ihvar , linvatr, lhvsr, lidht , lidrgr,

* lintf., Inoht, Ipar ,Ipgap ,Ipint .

* Iplav , lpr , Iqchff, lqchfo, Iqchfr.

* lqchro, lqwrx , Iradr , Iradrn, Irdhlo.

* Irdhlr, lrdhvo, Irdhvr, lrft, Irftn,

* Irlqlv, lrnd , lrndr, lroar, lrolr.

* Iromr , lrovr , Irpowf, Isigr, Isr

* 1stru , 1tcefn,1 tcefo, I twaen, I twaeo,

* Itween, Itweeo, Itchff, Itchfr, ltid.

* Itlr, ltinr

$c$

common/ptab/ Itsatr, Itssnr, ltvr,

* ltvnr , lvisir, lvisvr, lvlcr , lvizr ,

* Ivinzr, Ivolr, Ivver, Ivvzr, Iwatr,

* lzht

c-----ptsize is called by genpt, roddat, vsslpt, and all component

c----pointer tables (via dualpt for Id components). it sets a uniform

c-----length for all pointer tables via paraneter iptsiz.

parameter (iptsiz=300)

integer pt(iptsiz)

c--.--

equivalence $(p t(1), 1$ beta)

dimension fmax $(7), \operatorname{lok}(7,2)$ 
real maxfln

common/sscon/ cf, eps, epspow,

* fflw , fmax, maxfln, rpcf', rtwfp ,

* stime, tpowr

common/sicon/ ipovel, ipowr, isscut,

c

* lok ,ncores, nef, net, nopow

dimension ceoșlp $(40)$

common/tsatcn/ aeos14, ceos1, ceos2,

* ceos 3, ceoslp

common/tsatcn/igas, iliq

c

c

integer org

dimension tmp (10)

character 1 il

character*25 taf $(10)$

data taf/'inner-surface pressure ', 'outer-surface pressure

1

- inner-surface area

' ' 'outer-surface area

2 'inner and outer sf. areas', 'inner-surface th.conduct.'

3 'outer-surface th.conduct.', 'inner \& outer sf.th.cond.',

4 'wall thermal conductivity', 'sf.areas \& wall th.cond. '/

c

$c$

$c$

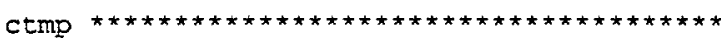

c

conv_t $=273.15$

c

ctmp

$c$

c

do 510 icmp=1, nhtstr

c

$1 \mathrm{cmhs}=i \mathrm{a}(1 \mathrm{cmhs}+i \mathrm{cmp}-1)$

call rdlcm ( $f 1 t$, lcmhs, lentbl)

$1 \mathrm{cmh}=1 \mathrm{cmhs}$

call $\mathrm{rrdlcm}$ ( $1 \mathrm{cmh}$ )

$c$

write (iout, 100)

100 format $\left(/ 1 x, 119\left({ }^{\prime}-{ }^{\prime}\right)\right)$

if (nstep.eq.0) then

$i=i c m p+n c o m p$

if (i.1t.10) then

numbn $1=11$

numbn2 $=11$

numbon $3=i$

else

if (i.1t.100) then

numbn $1=11$

numbn $2=i / 10$

numbn $3=i-$ numbn $2 * 10$

if (numbn 3 . eq. 0 ) numbn $3=10$

else

numbn $1=i / 100$

numbn2 $=(i-$ numbn $1 * 100) / 10$

numbn $3=(i-$ numbn $1 * 100)-$ numbn $2 * 10$

if (numbn2. eq. 0) numbn2 $=10$

if (numbn3.eq. 0) numbn $3=10$

endif

endif

call wrlcm(flt, lcmis, lentbl) 


\section{endif}

write (iout, 110) $\mathrm{nm}(1$, numbn1), $\mathrm{nm}(1$, numbn2), nm(1, numbn3), 1 $\operatorname{nm}(1, n u m b m 1), n m(1, n u m b m 2), n m(1, n u m b m 3)$

110 format $(1 x, 3(a 5,1 x), 84 x, 2(a 5,1 x), a 5)$

write (iout, 120$) \mathrm{nm}(2, \operatorname{numbn} 1), \mathrm{nm}(2, \operatorname{numbn} 2), \mathrm{nm}(2$, numbn 3$)$, etime, lus, 1 delt, lus, nstep, nm (2, numbm1), nm (2, numbm 2$), n m(2, n u m b m 3)$

120 format $(1 x, 3(a 5,1 x)$, 'time is ', f13.6,a,', time-step size is '.

$1 \quad f 11.6, a,{ }^{\prime}$, time-step number is ', is, $\left.1 \mathrm{x}, 2(\mathrm{a} 5,1 \mathrm{x}), \mathrm{a} 5\right)$

write (iout, 110) $\mathrm{nm}(3$, numbn1), $\mathrm{nm}(3, \operatorname{numbn} 2), \mathrm{nm}(3$, numbn 3$)$,

1 $\mathrm{nm}(3$, numbm1 1$), \mathrm{nm}(3$, numbm 2$), \mathrm{nm}(3$, numbm 3$)$ write (iout, 130) $\mathrm{nm}(4$, numbn1), $\mathrm{nm}(4$, numbn2), $\mathrm{nm}(4$, numbn 3$)$,

I ctitle, $\operatorname{nm}(4$, numband), nm (4, numbm2), nm (4, numbm3)

130 format $(1 x, 3(a 5,1 x), 26 x, 4 a 8,26 x, 2(a 5,1 x), a 5)$

write (iout, 110$) \mathrm{nm}(5$, numbn 1$), \mathrm{nm}(5$, numbn 2$), \mathrm{nm}(5$, numbn 3$)$,

1 $\mathrm{nm}(5$, numbm1 $), \mathrm{nm}(5$, numbom 2$), \mathrm{nm}(5$, numbm 3$)$

write (iout, 140)

140 format $\left(1 \mathrm{x}, 119\left({ }^{\prime}-'\right) /\right)$

write (iout, 150) type, num

150 format $/ /$ ' the heat-structure component type is a ', as,

I ' and the component number is', i4)

$\mathrm{c}$

if (nopowr.eq. 0 ) then

$\operatorname{tmp}(1)=$ rpower

if (ioout.eq.1) then

call uncnvt ('rpower', tmp, $1,1,-1$ )

endif

tpower $=\operatorname{tmp}(1)$

if (irpwty.ge.11) then

write (iout, 160) tmp (1), Iupw, rmckn, (a (1srp+i-1), $i=1,5)$

160 format (/' reactor-core power is',1p,e12.5,a, and neutron',

'multiplication constant keff is', Op, $f 9.6 / /$ ' prg',

'. sho is',f9.6,', tf.fdbk. tho is', f9.6,', tc.fabk',

'. rho is', f9.6,', vf. Edbk. tho is', f9.6,', sl.fdbk',

'. tho is', f9.6)

eise

if (ixpwty.It.5) then

write (iout, 170) tmp (1), lupw, rmekn

170 format (/' reactor-core power is',1p,e13.6,a, ' and neutron'

1 'multiplication constant keff is', 0p, f9.6)

else

write (iout, 180) tmp (1), lupw

180

format (/" total power generated is', 1p, e13,6,a) endif

endif

endif

c

call manage $(a,-$ nrods, lenrd, locrod, icp, -20)

do 460 ncr $=1$, nrods

c

call manage $(a$, ncr, org $, 1,1,2)$

ncraz $=i a($ lnoht + org)

norl $=$ ncr -1

$\mathrm{ni}=0$

no $=0$

if (idbci.eq.2) ni=ia(lidrod+ncrl)

if ((idbci.ne.2) and. (idbco.eq.2)) no=ia(lidrod+ncrl)

if ((idbci.eq.2) .and. (idbco.eq.2)) no=ia (lidrod+ncrl+nrods)

if (nopowr.ne.0) then

write(iout, 190) type, ncr, ni, no 


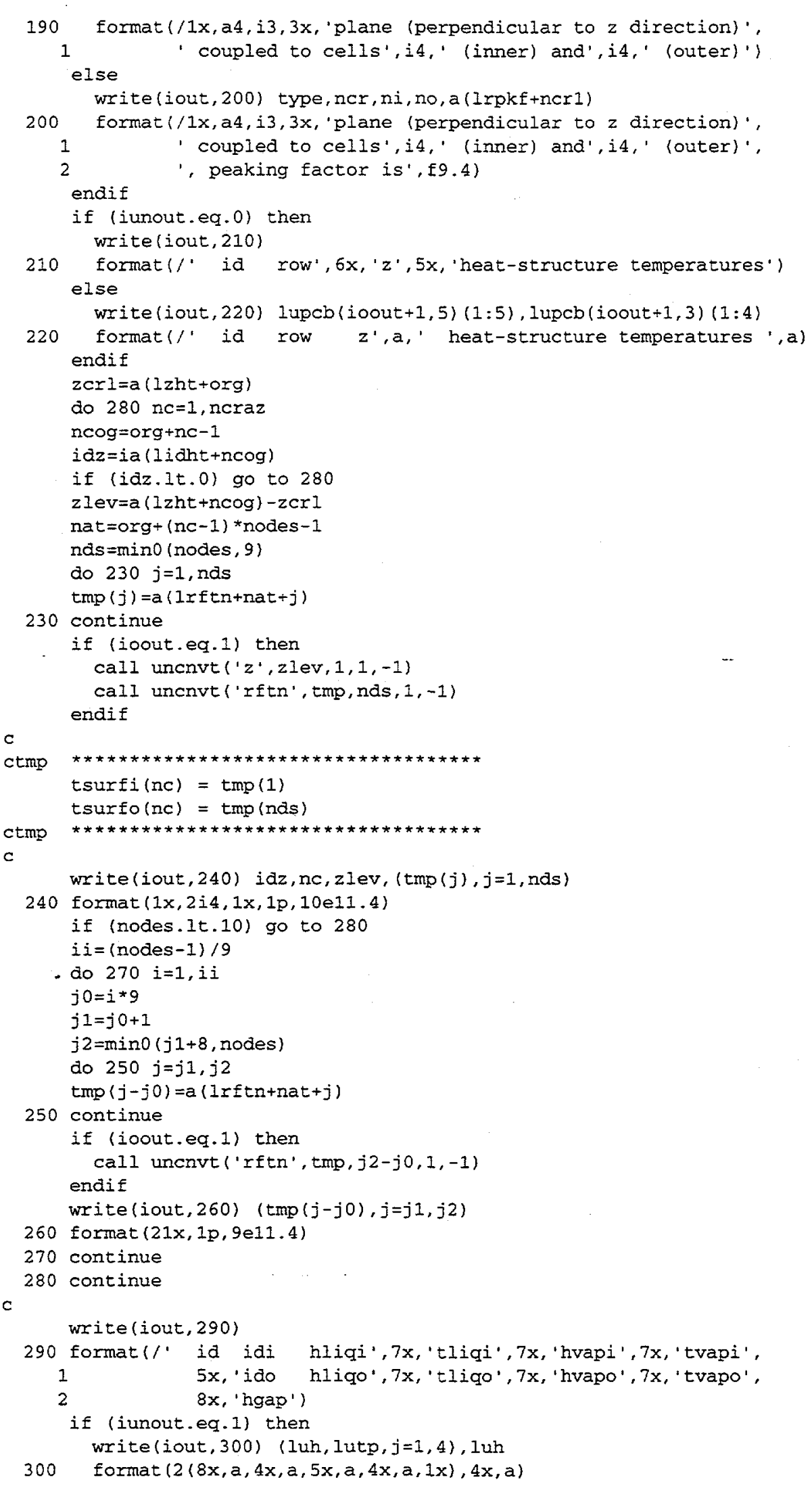


endif

$j b d=1$

del $z p=0.0$

do $370 \mathrm{nc}=1$, ncraz

$\mathrm{ncog}=$ org $\mathrm{n} \mathrm{nc}-1$

delzm=delzp

delzp=a $(1 z h t+\min 0(n \operatorname{cog}+1$, org $+n c r a z-1))-a(1 z h t+n c o g)$

rdzmp $=1.0 /($ delzm+delzp)

idz=ia (lidht + ncog)

if (idz.lt.0) go to 370

if (idbci.eq.2) then

$\operatorname{tmp}(1)=a(\operatorname{lh} f \mathrm{f}+\mathrm{ncog}+\mathrm{lndrd})$

$\operatorname{tmp}(3)=a(1 \mathrm{hrfv}+\mathrm{ncog}+\mathrm{lndrd})$

$\operatorname{tmp}(2)=\operatorname{arnax} 1(\operatorname{ceos} \operatorname{lp}(32), a(1 t \ln x+o r g+j b d+1 n d r d))$

tmp (4) $=\operatorname{amax} 1(\operatorname{ceoslp}(34), a(1$ tvnr + org $+j b d+\operatorname{lndrd})$ )

idhti $i=a($ liht $f+n c o g+\operatorname{lnd} d x)+0.01$

else

$\operatorname{tmp}(1)=\mathrm{hli}$

$\operatorname{tmp}(3)=$ hvi

$\operatorname{tmp}(2)=\operatorname{amax} 1(\operatorname{ceos} \operatorname{lp}(32), t 1 i)$

$\operatorname{tmp}(4)=\operatorname{amax} 1(\operatorname{ceos} I p(34), t v i)$

icht $i=0$

endif

if (idbco.eq.2) then

$\operatorname{tmp}(5)=a(1 h r f l+n c o g)$

$\operatorname{tmp}(7)=a(\operatorname{lhr} f \mathrm{v}+\mathrm{ncog})$

$\operatorname{tmp}(6)=\operatorname{amax} 1(\operatorname{ceoslp}\{32), a(1 t \ln r+o r g+j b d))$

tmp $(8)=\operatorname{amax} 1(\operatorname{ceosip}(34), a(1$ tvnr $+o r g+j b d))$

idhto $=a($ liht $f+n \operatorname{cog})+0.01$

else

$\operatorname{tmp}(5)=h l o$

$\operatorname{tmp}(7)=$ hvo

tmp $(6)=\operatorname{amax} 1(\operatorname{ceoslp}(32), t 10)$

$\operatorname{tmp}(8)=\operatorname{amax} 1(\operatorname{ceos} l p(34)$, tvo $)$

idhto $=0$

endif

if (idz.1t.1000) go to 310

$j \mathrm{bd}=i d z-1000$

if (idbci.eq.2) then

hdzm=a (Ihrll+org+jbd-1+indrd)*delzm

$h d z p=\operatorname{tmp}(1) * \operatorname{del} z p$

hdzmp=hdzm+hdzp

tmp (1) =hdzmp* rdzmp

if (hdzmp.gt.0.0) then

hdzm=hdzm*tmp (2)

hdzp=hdzp *amaxl (ceoslp (32) , a (ltlnr+org+jbd+lndrd))

$\operatorname{tmp}(2)=(h d z m+h d z p) / h d z m p$

endif

$h d z m=a(1 h r l v+o r g+j b d-1+1 n d r d) * d e l z m$

hazp $=t m p(3) \star d e l z p$

hdzmp=hdzm+hdzp

$\operatorname{tmp}(3)=h d z m p * r d z m p$

if (hazmp.gt.0.0) then

hdzm=hdzm*tmp (4)

$h d z p=h d z p * a \max 1(\operatorname{ceos} \mathrm{p}(34), a(1$ tumr+org $+j b d+$ Indrd $))$

$\operatorname{tmp}(4)=(h d z m+h d z p) / h d z m p$ endif

endif

if (idbco.eq.2) then

hdzm=a $(1 \mathrm{hr} 11+$ org $+j b d-1) * \mathrm{del} z \mathrm{~m}$

$h d z p=\operatorname{tmp}(5) * \operatorname{del} z p$

$h d z m p=h d z m+h d z p$ 


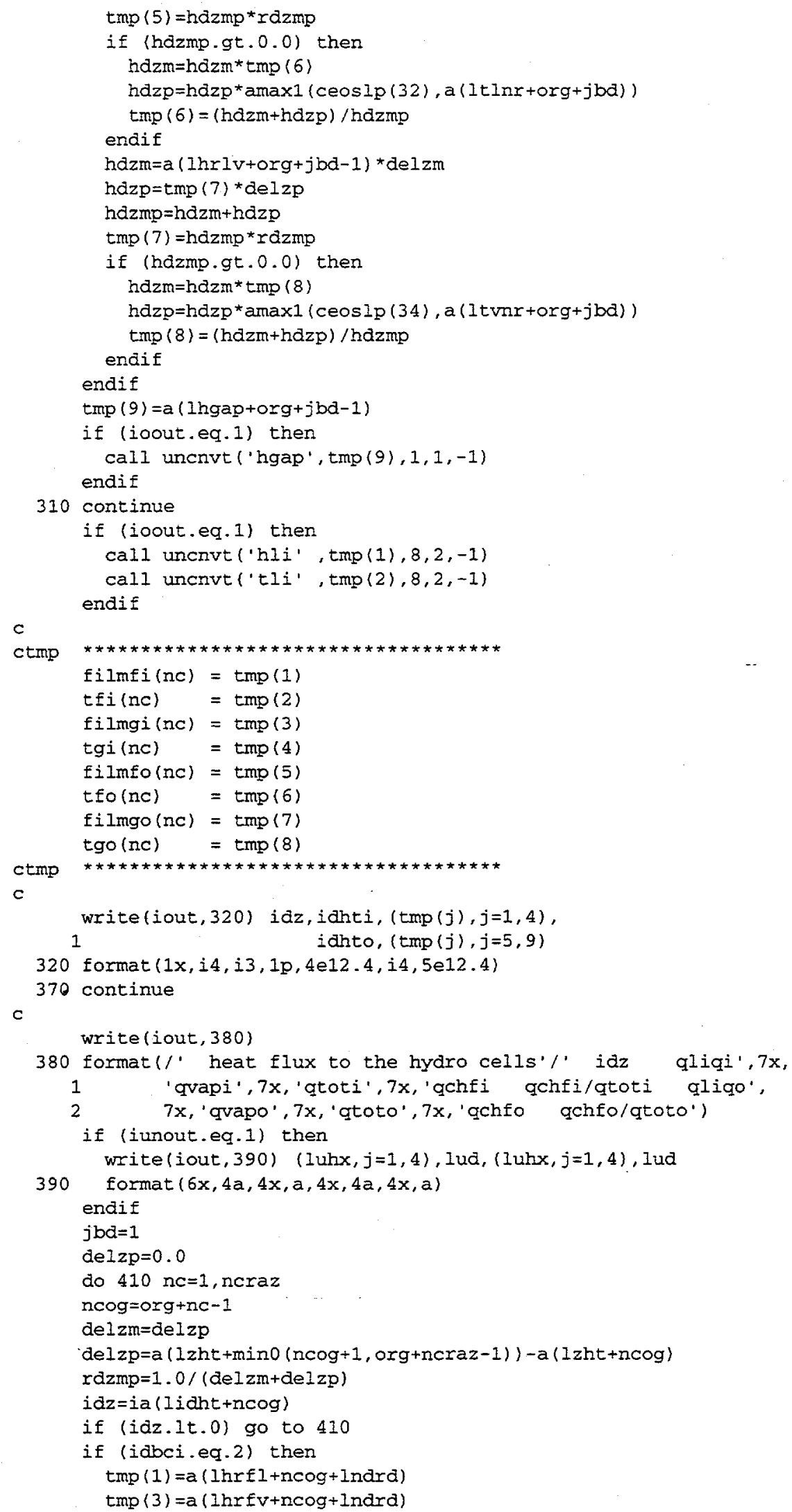


$\operatorname{tmp}(2)=\operatorname{amax} 1(\operatorname{ceos} 1 p(32), a(1 \mathrm{t} \ln r+o r g+j b d+1 n d r d))$

$\operatorname{tmp}(4)=\operatorname{amax} 1(\operatorname{ceoslp}(34), a(1$ tvnr $+o r g+j b d+1$ ndrd $))$

qchf $i=a(1$ qch $f+o r g+j b d-1+1$ ndrd $)$

else

$\operatorname{tmp}(1)=h 1 i$

$\operatorname{tmp}(3)=\mathrm{hvi}$

$\operatorname{tmp}(2)=\operatorname{amax} 1(\operatorname{ceos} 1 p(32), t l i)$

$\operatorname{tmp}(4)=\operatorname{amax} 1(\operatorname{ceos} \operatorname{lp}(34), \mathrm{tvi})$

qch $i=0.0$

endif

if (idbco.eq.2) then

tmp (5) =a (lhrfl+ncog)

$\operatorname{tmp}(7)=a(\operatorname{lhrfv}+\mathrm{ncog})$

$\operatorname{tmp}(6)=\operatorname{amaxl}(\operatorname{ceoslp}(32), a(1 t \ln r+o r g+j b d))$

tmp (8) =amaxl (ceoslp (34), a (ltvnr+org $+j b d)$ )

qchfo=a ( 1 qchfr + org $+j b d-I$ )

else

$\operatorname{tmp}(5)=\mathrm{hlo}$

$\operatorname{tmp}(7)=$ hvo

tmp $(6)=\operatorname{amax} l(c e o s l p(32), t l o)$

tmp (8) $=\operatorname{amax} 1(\operatorname{ceos} l p(34)$, tvo $)$

$q \mathrm{ch} f \mathrm{o}=0.0$

endif

if (idz.1t.1000) go to 395

$j b d=i d z-1000$

if (idbci.eq.2) then

hdzm=a (lhrll+org+jbd-1+Indrd $) * d e l z m$

hdzp $=\operatorname{tmp}(1) * d e l z p$

hazmp=hdzm+hdzp

$\operatorname{tmp}(1)=h d z m p * r d z m p$

if (hdzmp.gt.0.0) then

hdzm=hdzm*tmp (2)

$h d z p=h d z p * \operatorname{amax} 1(\operatorname{ceos} l p(32), a(1 t \ln r+o r g+j b d+1 n d r d))$

$\operatorname{tmp}(2)=(h d z m+h d z p) / h d z m p$

endif

$h d z m=a(1 h r l v+o r g+j b d-1+1 n d r d) * d e l z m$

hdzp $=\operatorname{tmp}(3) *$ delzp

hdzmp=hdzm+hdzp

$\operatorname{tmp}(3)=h d z m p * r a z m p$

if (hdzmp.gt.0.0) then

hdzm=hdzm*tmp (4)

$h d z p=h d z p * a \max 1(\operatorname{ceos} l p(34), a(1 t v n r+o r g+j b d+l n d r d))$

$\operatorname{tmp}(4)=(h d z m+h d z p) / h d z m p$

endif

gchfi $=(q c h f i * d e l z m+a(\operatorname{lqchf} r+o r g+j b d-1+\operatorname{lndrd}) * \operatorname{delzp}) * r d z m p$ endif

if (idbco.eq.2) then

hdzm=a $(1 \mathrm{hr} 11+$ org $+j \mathrm{bd}-1) * d e l z m$

hdzp $=\operatorname{tmp}(5) *$ delzp

hazmp=hdzm+hdzp

$\operatorname{tmp}(5)=h d z m p * r d z m p$

if (hdzmp.gt.0.0) then

hdzm=hdzm*tmp (6)

$h d z p=h d z p * \operatorname{amax} 1$ (ceoslp (32), a ( It lnr+org+jbd))

$\operatorname{tmp}(6)=(h d z m+h d z p) / h d z m p$

endif

hdzm=a $(1 \mathrm{hrlv}+$ org $+j b d-1)$ *delzm

hdzp $=\operatorname{tmp}(7) *$ del $z p$

hdzmp=hdzm+hdzp

$\operatorname{tmp}(7)=$ hdzmp ${ }^{*}$ rdzmp

if (hdzmp.gt.0.0) then

$h d z m=h d z m * t m p(8)$ 


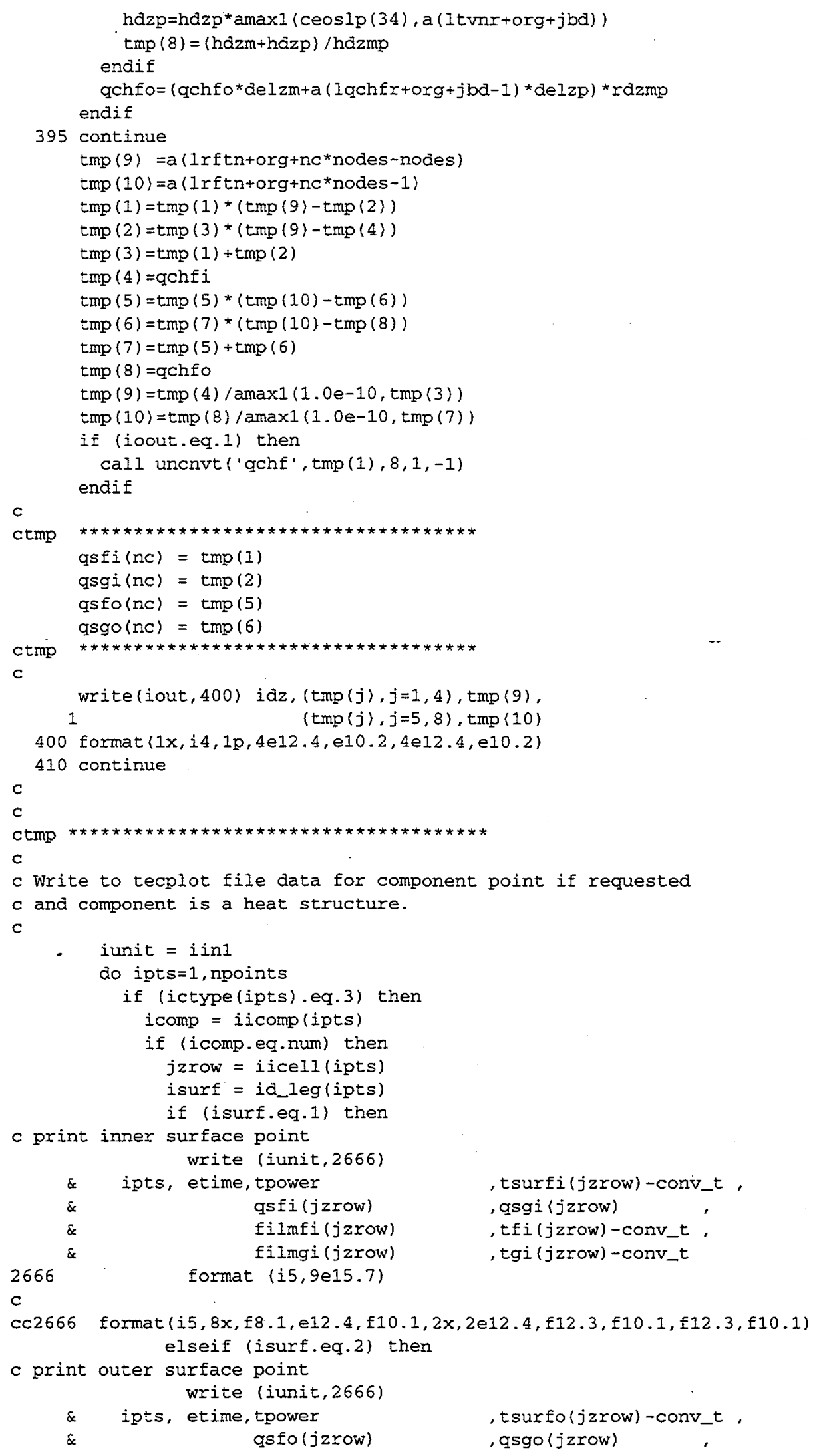




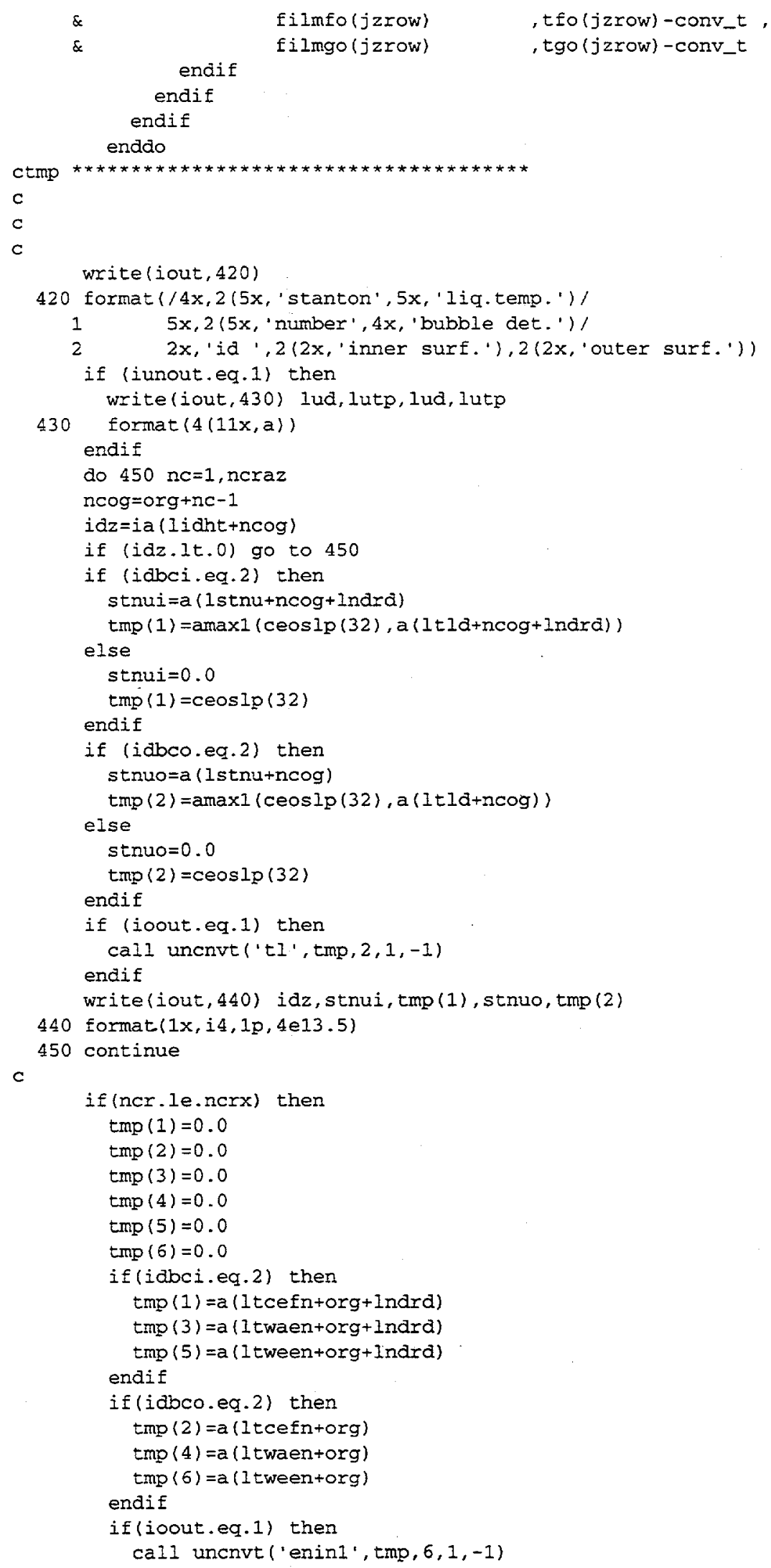




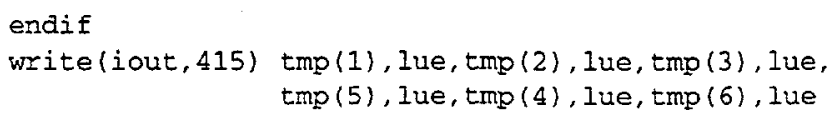


c return end

\section{Fortran file wpipe.f:}

subroutine wpipe(cfmass, cener, tsec) 
* lcmptr, levstg, llvflg, ncmn , nomx

* ncontr, nconts, ncontt, nerg ,ndial ,nencl,

* newrfa, nfrc1, nfrc3, nitav, nitmn,

* nitmx ,nloopp, nosets, nsend, nseo ,

* nsma , nsmx , nspl , nspu , nsso ,

* nstab, nstp , nvgrav, nvpow , oitmax, sitmax,

* stdyst, transi, motsm, statsm, saxsm

integer dstep, oitmax, sitmax, stdyst,

* transi, statsm, saxsm

c fixed length table

integer fit(1)

real rflt(1)

equivalence (flt(1), rflt(1))

character type*8, title*32, ctitle*8

common/Eltab/ htlsci,htlsco, coild, pinteg

comon/fltab/ ctitle(4),icflg, id

* irest, lenarr, lenfv, lenfv2, lenptr.

* lenvit, lextra, Ifv , lfvn, ncellt,

* numbmi, numbm2, numbm3, numbn1, numbon2.

* numbn3, nodes, num , nrvlt, ctype

equivalence (flt (1), htlsci), (type, ctype), (ctitle(1), title)

c

common/units/ ibfadd, ibfadg, ibfadr,

* ibflnd, ibflng, ibflnr, idout, ieeeg, igout,

* ikeyba, imout, in ,inlab, inproc,

* ioall, iodone, ioerr, iogrf, ioinp.

* iolab , ioout , ioskip,iout ,irstrt,

* itty , iunlab, iunout, lcmcpa, nittab,

* npwtab, nrdy

common / cunits/card

c character $\star 100$ card

common/istat/ varerm, verr

common/istat/ iott, nstep, oitno

integer oitno

c

common/labelv/factor (150), offset (150)

common/labelv/ih (26), itls (777), itsv(105),

1

ils, ilu, ilun, iold

- common/labelv/labels (777), labsv $(2,105), 1$ abun (150),

1 luncb $(2,150), \operatorname{lupcb}(2,150), \operatorname{runcb}(2,150)$

common/labelv/alpbet, Iuar, 1ucp, 1ud, ludh, lue, luen,

1 Iuh, Iuha, Iuhx, luid, Iuis, Ium, Iunf, Iup,

2 Iupd, Iuph, lupt, lupw, Iur, Ius, lusp, Iusz,

3 Iut, Iutc, Iutm, Iutp, Iuv, Iuvf, Iuvo, Iuz

character*2 lud, lutp, lus

character*3 lut, luz

character*4 luar, Iue, Ium, Iuvo

character $* 5$ lup, Iupd, Iuv, Iuvf

character $* 6$ luis

character*7 luen, lumf, lupt, Iupw

character $* 8$ labels, labun, luid, lur, Iusp, Iusz, Iutm

character*9 Iuha

character $* 10$ lucp

character*11 luph

character*12 luhx, lutc, runcb

character $* 13$ luh, Iuncb, lupcb

character*14 labsv

character $* 19$ Iudh

character ${ }^{2} 6$ alpbet 
dimension $f I(2), f v(2)$

common/vltab/ aal111,bsmass, cpow, eninp, epsw ,

* 11

, fv houtl, houtv, plent

* powin, powoff, qint, gout, gp 3 in,

* qp3off, radin, rpowmx, rqp $3 \mathrm{mx}$, th

* toutl, toutv, vflow, $z$

* z11111

common/vltab/ ia1111,iacc, icht, icj1,

* icj2, icone, ionoff,ipf ,ipow

* ipowsv, ipowtr, ipp , iqf , iqp

* iqp3sv, iqp 3 tr, isollb, isolrb, js 1

* js2 ,jun1, jun2, ncells,

* nonoff, npowrf, npowsv, npowtb, nop3rf,

* nqp3sv, nqp3tb, type1 , type2 ,

* zi1111 integer vit, type1, type2, zil111

c-----condeck vitaim sets an upper limit to all variable length tables c----via parametex ivltsz. condeck genvlt calls vltdim directly, thus c----providing all variable length tables with a uniform length.

c----parameter ivltsz is used to set a uniform length to all

c----variable length tables.

parameter (ivltsz=270)

dimension vlt(ivltsz)

equivalence ( $\mathrm{v} 1 \mathrm{t}(1)$, aal111)

c-----

$\mathrm{c}$

c

c

dimension tmp (3)

c

write(iout, 100) num, jun1, jun2

100 format // the component type is a pipe, component number is',

1 i4,', first junction number is',i4,', and second',

2 ' junction number is', i4)

cEmass $=0.0$

cener $=0.0$

c

if Igw $=1$

itee $=0$

call ecomp (num, 1, ncells, iflgw, nodes, cfmass, pinteg, cener, tsec,

$\&$

- itee)

c

if (ipow.eq.1) then

$\operatorname{tmp}(1)=$ cpow

$\operatorname{tmp}(2)=e n i n p+d e l t *$ cpow

if (ioout.eq. 1 ) then

call uncnvt ('cpow', $\operatorname{tmp}(1), 1,1,-1)$

call uncnvt ('eninp', tmp $(2), 1,1,-1)$ endif

write(iout, 110) tmp(1), Iupw, tmp (2), lue

110 format (/' power deposited in the pipe coolant is', Ip,e13.5,a,

1 'and total energy deposited in the pipe coolant is',

2 e13.5, a)

endif

$\mathrm{c}$

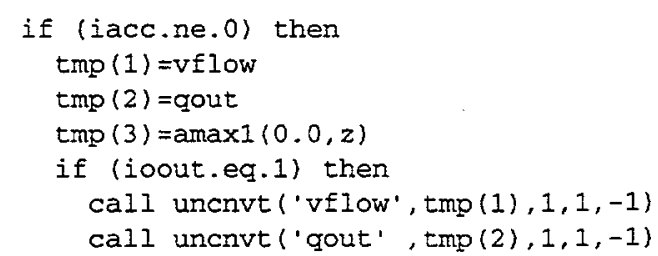




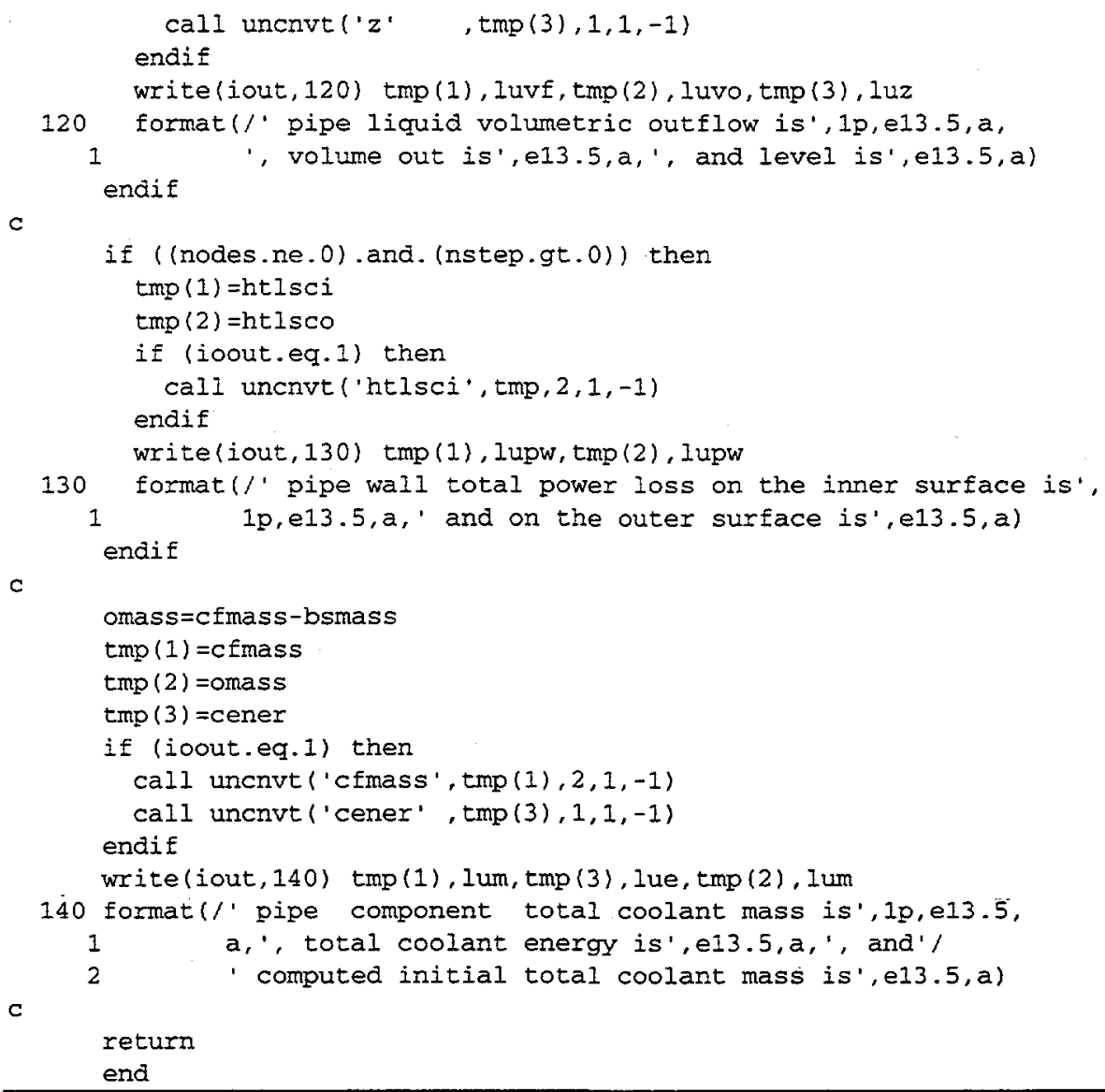

\section{Fortran file wplen.f:}

subroutine wplen (cfmass, cener, bd, lenbdd, tsec)

subroutine wplen writes thermal-hydraulic solution parameters to file trcout for the plenum component

common/contrl/ cpuflg, dammc, damx , delt,

* deltht, difmin, dprmx , dt lmx ,dtmax ,

* atmin ,dto ,dtrat ,atrmx ,atsmx ,

* dtvmx ,encmax, eps1 ,eps2, epso.

* ercemx,epss, etime ,htlosi,htloso,

* odelt, powerc,pssmn ,pssmx , rfat ,

* rvmax ,tend , tercmx, timec ,timet.

* tmmax, varer, vcmn , vcmx, vmaxo,

* vmaxt, vmaxt3, vmcor , vmnew, vmold,

* vmxt30,xtable,x0sm, y0sm, z0sm ,

* omsasm,wsasm, wỏsasm, t0sm (3), xvsm ,

* yvsm, zvsm, $\operatorname{rmatsm}(3,3), \operatorname{omsm}(3), \operatorname{wsm}(3)$,

* $\operatorname{wdsm}(3), \operatorname{errsm}, d t s m$

common/contrl/ dstep, iadded, iblaus, iccmx ,

* icmp , icmpmx, icp , idiag, idiags, ieccpx, ieos, iff3d,

* ifprep, igeom3, im100, im100x, imfr ,

* invan , iofftk, ipak , ipak3d, ipakon, ipkpmp,

* ireset, irsflg, irstfl, isolut, issflg, istdy ,

* isttc, ithd, itmin, itpako, jfat , kccmx ,

* lcmptr, levstg, livflg, ncmn, , ncmx

* ncontr, nconts, ncontt, nerg , ndial , nencl, 
* newrfa, nfrc1, nfrc3, nitav, nitmn,

* nitmx, nloopp, nosets, nsend, nseo ,

* nsmn , nsmx , nspl , nspu , nsso ,

* nstab, nstp , nvgrav, nvpow , oitmax, sitmax,

* stdyst, transi, motsm, statsm, saxsm

integer astep, oitmax, sitmax, stayst,

* transi, statsm, saxsm

common/istat/ varerm, verr

common/istat/ iott, nstep, oitno

integer oitno

c

common/labelv/factor (150), offset (150)

common/labelv/in (26), itls (777), itsv(105),

ils, ilu, ilun, iold

common/labelv/labels (777), labsv (2, 105), labun (150),

$1 \operatorname{luncb}(2,150), \operatorname{lupcb}(2,150), \operatorname{runcb}(2,150)$

common/labelv/alpbet, luar, Iucp, lud, Iudh, lue, luen,

I Iuh, Iuha, Iuhx, Iuid, Iuis, Ium, lumf, Iup,

2 Iupd, luph, lupt, lupw, lur, Ius, lusp, 1usz,

3 Iut, Iutc, Iutm, lutp, luv, luvf, luvo, luz

character*2 lud, lutp, lus

character*3 lut, luz

character*4 luar, Iue, Ium, luvo

character*5 Iup, lupd, luv, luvf

character*6 Iuis

character*7. Iuen, lumf, Iupt, Iupw

character*8 labels, labun, Iuid, lur, Iusp, lusz, Iutm

character*9 luha

character*10 lucp

character*11 Iuph

character*12 luhx, lutc, runcb

character*13 Iuh, luncb, Iupcb

character*14 labsv

character*19 Iudh

character*26 alpbet

$c$

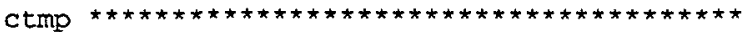

c

common /tecplot/iicomp (500), iicell(500), ictype (500),

\& . id_leg(500), npoints, inn

c

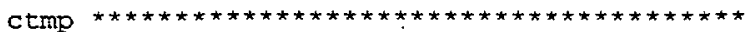

C

plenum data

parameter (ifxsiz=5000000)

common a (ifxsiz)

dimension alcm(1)

equivalence (a(1), alcm(1))

dimension ia (ifxsiz)

character* 8 ach (ifxsiz)

equivalence (a(1), $\operatorname{ach}(1)$ )

equivalence $(a(1)$, ia(1))

$\mathrm{c}$

common/dimen/ ifree, jnvssl,kvelit,

* kvel2t, kvel3t, last , laim, leniod, lendim,

* lentbl, Ifree , 1last, Imldp , Inldpv, Inrapt,

* locrap, 1start, lstrap, lver , maim,

* memfig, moff, ncomp, ncompt,

* nhtstr, njnmx , njnt , njun , nloops,

* nmvssl,npx ,nstgj, nthm ,nthmla, 


\section{TRAC CODE MODIFICATIONS}

Page:

* nthm3d, numtcr, nvcon, nvelx ,nvely ,

* nvelz , nwrda

c

common/units/ ibfadd, ibfadg, ibfadr,

* ibflnd, ibfing, ibflnr, idout, ieeeg, igout.

* ikeybd, imout, in ,inlab ,inproc,

* ioall, iodone,ioerr, iogrf , ioinp .

* iolab, ioout, ioskip, iout ,irstrt,

* itty , iunlab, iunout, lcmcpd, nittab.

* npwtab, nray

common /cunits/cara

character $* 100$ card

c

common/junct/jptr,jmatch

c

c fixed length table

integer flt (1)

real rflt (1)

equivalence (flt (1), rflt(1))

character type 8, title* 32, ctitle* 8

common/fltab/ htlsci,htsco,coild, pinteg

comon/fltab/ ctitle(4),icflg, id

* irest, lenarr, lenfv, lenfv2, lenptr,

* lenvit, lextra,lfv, ifvn, ncellt,

* numbm1, numbm2, numbm3, numbn1, numbn2,

* numbr 3 , nodes , num , nrvlt , ctype

equivalence (flt(1), htlsci), (type, ctype), (ctitle(1), title)

$c$

dimension $\operatorname{ld}(3), \operatorname{Idn}(3)$

common/ptab/ lalp, lalpn, lalpd,

* lalpan, lalv , lalvn, lalve , lalven,

* lara , laran , larel , lareln, larev,

* larevn, lard, larln, larv , larvn,

* Ibit , lbitn , lchti , lchtin, Ichtia,

* lchtan, lcif , lcifn, lcone, lconcn,

* Id, lan, lea , lean, lel

* leln, lev, levn, Igam, Igamn,

* Ihig, Ihigo, Ihil , Ihilo, Ihiv

* Inivo, lp , Ipn , Ipa ,lpan

* lqppc, Iqppco, lroa , Iroan, Irol

* Iroln, lrov, Irovn i is , Isn

* Itce, Itcen, Ita , Itan, itl

* Itln, itv ,Itvo , Itw , ltwn

* Itwa , Itwan. Itwe Itwen , Ivi ivin,

* Ivlt , Ivito, Ivm , Ivmn, Ivv ,

* Ivvr ivvt ivvto

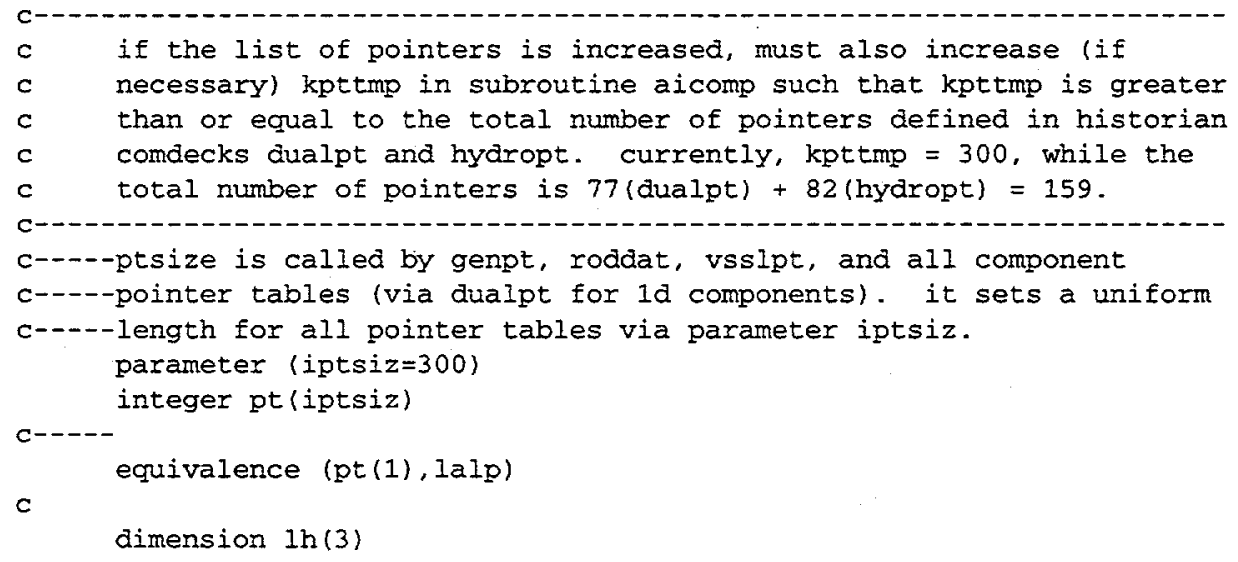


common/ptab/ lalpmn, lalpmx, lalpo

* lam , lare, lefz . lcl, lcpl,

* lcpv, lcv, Idalva, ldfldp, Idfvap.

* lariv, lox , lelev, Ifa , Ifavol,

* Ifinan, Ifric, Ifsmlt, Igrav, Igrvol,

* In , Ind , Ihdhe, Ihfg, Ihgam.

* Ihla Ihlatw, Ihva ,Ihvatw,

* Iqri , lqrv , lop 3 .

* Igppp ,Iregnm, Irhs ,Irmem, Irmvm ,

* lrarl, lrarv ,

* Ixsm, Iysm, Izsm, Irsm, Irosm,

* Infvsm, Inflsm, luvsm, Infcvsm, Infclsm, 1vvsm, 1vlsm,

* Inf1sm, lnf2sm, $1 \mathrm{nf} 3 \mathrm{sm}, \ln f v 4 \mathrm{sm}, \operatorname{lnf} 14 \mathrm{sm}$,

* Irom , Irvmf, lsig , Itria, Itsat.

* Itssn , 1visl , lvisv , lvlalp, lvlvc,

* lvlvol, lvlx , lvol , lvr , Ivrv.

* Ivvvol, lvvx , Iwa , lwat ,lwfl,

* lwfv , lwfmfl, lwfmfv

$c$ if the list of pointers is increased, must also increase (if

c

c

c

c

c

$c$

c

necessary) kpttmp in subroutine aicomp such that kpttmp is greater than or equal to the total number of pointers defined in historian comdecks dualpt and hydropt. currently, kpttmp $=300$, while the total number of pointers is 77 (dualpt) +82 (hydropt) $=159$.

in case new pointers are added after lwfmfv, then the coding in subroutine aicomp (third executable statement) for evaluation of the local variable lenpt 1 = locf (lwfmfv) - locf (pt(1)) + 1 must be changed to use the name of the new pointer at the end of this section of the common block / ptab /.

common/ptab/ lalw, lavw, labnd,

* Idnfl, Idnfv, Iioj, Ijsn, Ijunj,

* Ipak, Isgn, lzzzzz

common/vltab/ aall11,bl ,bsmass,

* bv ,epsw, fasl,fas2, flxa,

* flxal, flxav, flxc , Elxel, flxev,

* $1 \mathrm{IxI}, \mathrm{flxv}, \mathrm{rxcl}, \mathrm{rxcv}, \mathrm{xl}$,

* xv, 211111

common/vltab/ ia1111,k ,iconc,

* ipow ,juns1 ,juns2, ncells, npljn,

* zi1111

integer vlt, zil111

c----comdeck vltdim sets an upper limit to all variable length tables c----via parameter ivltsz. comdeck genvlt calls vltaim directly, thus c----providing all variable length tables with a uniform length.

c----parameter ivitsz is used to set a uniform length to all

c----variable length tables.

parameter (ivltsz $=270$ )

dimension vlt(ivltsz)

equivalence (vlt(1), aa1111) c-- - -

c

\begin{tabular}{|c|c|c|c|c|c|c|}
\hline \multicolumn{2}{|c|}{ dimension } & $\operatorname{alp}(1)$ &, $\operatorname{alv}(1)$ & , alve (1) &, $\operatorname{ara}(1)$ & , arel (1) \\
\hline 1 & $\operatorname{arev}(1)$ &, $\operatorname{arl}(1)$ &, $\operatorname{arv}(1)$ & , bor $(1)$ &, $\operatorname{eva}(1)$ & , el (1) \\
\hline 2 & $\operatorname{ev}(1)$ &, $\operatorname{chti}(1)$ & , chtia(1) &, $\operatorname{gam}(1)$ & $p(1)$ & , pa (1) \\
\hline 3 & rova (1) &, $\operatorname{rol}(1)$ & $\operatorname{rov}(1)$ & , solid(1) &, $\operatorname{tI}(1)$ &, $\operatorname{tv}(1)$ \\
\hline 4 & bit $(1)$ & $\operatorname{hil}(1)$ &, $\operatorname{hiv}(1)$ & , & & \\
\hline 5 & $\operatorname{alpn}(1)$ & , alvn(1) & , alven (1) &, $\operatorname{aran}(1)$ & , areln (1) & , arevn (1) \\
\hline 6 & $\operatorname{arln}(1)$ &, $\operatorname{arvm}(1)$ &, $\operatorname{born}(1)$ & , evan (1) &, $\operatorname{eln}(1)$ &,$e \operatorname{m}(1)$ \\
\hline
\end{tabular}




$$
\text { cl (1) }
$$

alpo(1), am(1)

$$
, \operatorname{cpl}(1)
$$

, $\operatorname{arc}(1)$

, $\operatorname{cpv}(1)$

, cv (1)

$\operatorname{sig}(1), \operatorname{tsat}(1)$

, $\operatorname{tssn}(1)$

, hfg (1)

, dalp (1)

rom

wa (1), elev(1), gp3f(1), dalva(1), dxvol(1), favol (1) '

vlvol (1), vrvol(1), gravol(1), alpmn(1), alpmx (1), vlvc(1) ,

- fasmlt(1), vlalp (1),

$\operatorname{dtsap}(1), \operatorname{deldp}(1), \operatorname{devap}(1), \operatorname{deldt}(1), \operatorname{devat}(1), \operatorname{arolp}(1)$, drovp (1) , drolt(1), arovt(1), hvst(I), hlst (I) , dhvsp (1) , dhlsp (1), dtssp (1), devat (1), devap (1), $\operatorname{arvap}(1), \operatorname{drvat}(1)$, hgam(1), hla(1), hva(1), hlatw(1), hvatw(1), finan(1) , $1 \quad \operatorname{wat}(1), \operatorname{vrvpl}(1), x \sin (1), y \operatorname{sm}(1), z \operatorname{sm}(1)$

c equivalence (a(01),alp(1)), (a(02),alv(1)) , 1 (a(03),alve(I));(a(04), ara(1)), (a(05), arel(1)), $2(a(06), \operatorname{arev}(1)),(a(07), \operatorname{ar} 1(1)),(a(08), \operatorname{arv}(1))$, $3(a(09), \operatorname{bor}(1)),(a(10), \operatorname{eva}(1)),(a(11), e 1(1))$, $4(a(12), \operatorname{ev}(1)),(a(13), \operatorname{chti}(1)),(a(14), \operatorname{chtia}(1))$, $5(\mathrm{a}(15), \operatorname{gam}(1)),(\mathrm{a}(16), \mathrm{p}(1)),(\mathrm{a}(17), \mathrm{pa}(1))$, $6(a(18), \operatorname{rova}(1)),(a(19), \operatorname{rol}(1)),(a(20), \operatorname{rov}(1))$, 7 (a(21), solid(1)),(a(22),tl(1)) , (a(23), tv(1)) , $8(a(24), \operatorname{bit}(1)),(a(25), \operatorname{hil}(1)),(a(26), \operatorname{hiv}(1))$ equivalence (a(27),alpn(1)), (a(28),alvn(1)), $1(a(29), \operatorname{alven}(1)),(a(30), \operatorname{aran}(1)),(a(31), \operatorname{areln}(1))$, $2(a(32), \operatorname{arevn}(1)),(a(33), \operatorname{arln}(1)),(a(34), \operatorname{arvn}(1))$, $3(a(35), \operatorname{born}(1)),(a(36), \operatorname{evan}(1)),\left(a(37), e \ln (1)^{-\cdots}\right)$, 4 (a(38), evn(1) ), (a(39), chtin(1)), (a(40), chtan(1)), 5 (a(41), gamn(1)), (a(42),pn(1)) , (a(43),pan(1)) , $6(a(44), \operatorname{rovan}(1)),(a(45), \operatorname{roln}(1)),(a(46), \operatorname{rovn}(1))$, $7 \quad(a(47), \operatorname{solion}(1)),(a(48), \operatorname{tin}(1)),(a(49), \operatorname{tvn}(1))$, $8(a(50), \operatorname{bitn}(1)),(a(51), \operatorname{hiln}(1)),(a(52), \operatorname{hivn}(1))$ equivalence $(a(53), c l(1)),(a(54), \operatorname{cpl}(1))$, $1(a(55), \operatorname{cpv}(1)),(a(56), \operatorname{cv}(1)),(a(57), a) p o(1))$, $2(a(58), \operatorname{am}(1)),(a(59), \operatorname{arc}(1)),(a(60), \operatorname{hfg}(1))$, $3(a(61), \operatorname{dalp}(1)),(a(62), \operatorname{rom}(1)),(a(63), \operatorname{sig}(1))$, $4(a(64), \operatorname{tsat}(1)),(a(65), \operatorname{tssn}(1)),(a(66), v i s 1(1))$, $5(a(67), \operatorname{visv}(1)),(a(68), \operatorname{vol}(1)),(a(69), w a(1))$, 6 (a(70), elev(1)),(a(71), gpjf(1)), (a(72), dalva(1)). $.7(a(73)$, dxvol (1)), (a (74), favol(1)), (a(75), vivol(1)), $8(a(76), \operatorname{vvvol}(1)),(a(77), \operatorname{gravol}(1)),(a(78), a 1 p m n(1))$, 9 (a(79),alpmx(1)),(a(80),vlvc(1)), (a(81), fasmlt(1)) equivalence (a(82),vlalp(1)), (a(83), atsdp(1)), 1 (a(84), deIdp (1)), (a(85), devap(1)), (a(86), delat (1)), 2 (a(87), devdt(1)),(a(88), arolp(1)), (a (89), drovp(1) ), 3 (a(90), drolt (1)), (a(91), arovt (1)), (a (92), hvst(1)), 4 (a(93), hlst(1)), (a (94), ahvsp(1)), (a (95), dhlsp(1)), 5 (a(96), dtssp(1)),(a(97), devat (1)), (a(98), devap(1)), 6 (a(99), drvap(1)), (a(100), drvat(1)), (a(101), hgam(1)), 7 (a(102),hla(1)),(a(103),hva(1)), (a(104), hlatw(1)), 8 (a(105), hvatw(1)), (a(106), finan(1)), (a(107), wat(1)), $9 \quad(a(108), \operatorname{vrvpl}(1)),(a(109), x \operatorname{sm}(1)),(a(110), y \operatorname{sm}(1))$, $1 \quad(a(111), z \sin (1))$

c

c common/twostp/ npsfe, npsme, ntsprn

C dimension bd(lenbdd, 1), tmp (8)

$c$ ctmp $c$ 


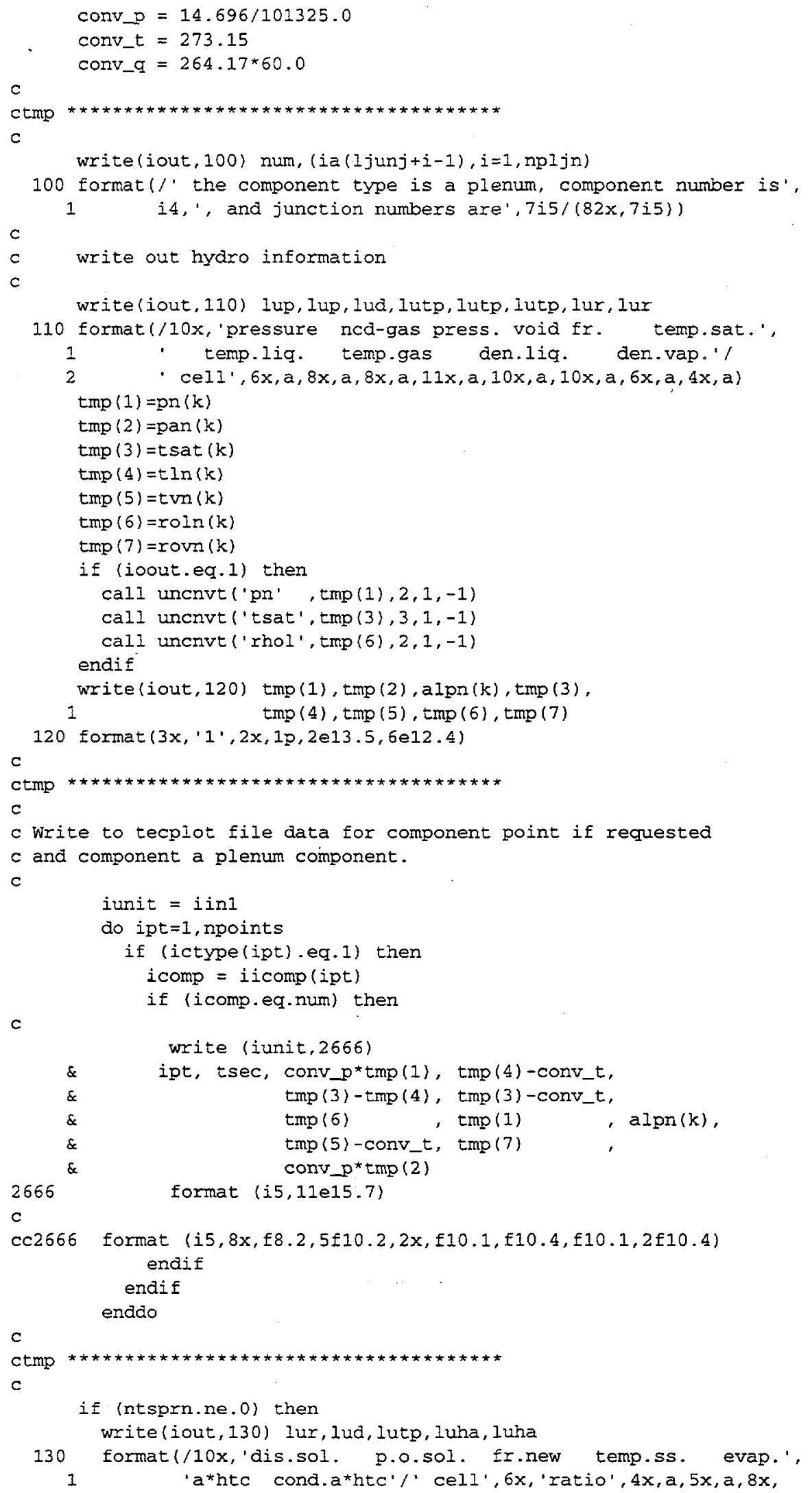


2 $\operatorname{tmp}(1)=\operatorname{soIidn}(k)$

$\operatorname{tmp}(2)=\operatorname{tssn}(k)$

$\operatorname{tmp}(3)=$ alve $(k)$

$\operatorname{tmp}(4)=\operatorname{cht}$ in $(k)$

if (ioout.eq.1) then

call uncnvt('sn' , $\operatorname{tmp}(1), 1,1,-1)$

cal1 uncnvt ('tssn', tmp (2), $1,1,-1$ )

call uncnvt('alve', $\operatorname{tmp}(3), 2,1,-1)$

endif

write(iout, 140) born (k), tmp (1), dalp (k), tmp (2), tmp (3), tmp (4)

format ( $3 \mathrm{x},{ }^{\prime} 1$ ' $, 2 \mathrm{x}, 1 \mathrm{p}, 1 \mathrm{e} 13.5,2 \mathrm{x}, 0 \mathrm{p}, \mathrm{f} 7.3,1 \mathrm{p}, 4 \mathrm{e} 11.4$ )

write (iout, 150) (Iuv, $i=1,8$ )

150 format (/16x, 'junction velocities (negative in to the plenum',

1 ' cell and positive out of the plenum cell)' $/ 4(8 x$,

$i i=(\operatorname{mpljn}+3) / 4$

vel.1ig. vel.gas ')/4(' jun.', $3 x, a, 7 x, a, 4 x)$

do $180 i=1, i i$

$j 0=(i-1) * 4$

$j 1=j 0+1$

$j 2=\min 0(j 1+3, \operatorname{npljn})$

$j 3=j 0+j 0-j 2$

do $160 j=j 1, j 2$

is $=i a(1 j s n-1+j)$

$\operatorname{tmp}(j-j 0)=b d(6, i s) * b d(23$, is $)$

$\operatorname{tmp}(j-j 3)=b d(6$, is $) * b d(24$, is $)$

160 continue

if (ioout.eg.1) then

call uncnvt ('vl', tmp, j2-j3,1,-1)

endif

write (iout, 170) (ia (1junj+j-1), tmp $(j-j 0), \operatorname{tmp}(j-j 3), j=j 1, j 2)$

170 format $(1 \mathrm{x}, 4(\mathrm{i} 4,1 \mathrm{p}, 2 \mathrm{e} 12.4,2 \mathrm{x}))$

180 continue

write (iout, 190) (Iuv, $i=1,8$ )

190 format(/13x, 'junction tilde velocities (negative in to the',

2

do $210 i=1, i j$

- plenum cell and positive out of the plenum cell)'/4(8x,

'velt.Iiq. velt.gas ')/4(' jun.', $3 x, a, 7 x, a, 4 x)$ )

* $j 0=(i-1) * 4$

$j 1=j 0+1$

$j 2=\min 0\langle j 1+3, n p I j n\rangle$

$j 3=j 0+j 0-j 2$

do $200 j=j 1, j 2$

is $=i a(1 j \operatorname{sn}+i-I)$

$\operatorname{tmp}(j-j 0)=b d(6, i s) * b d(62$, is $)$

$\operatorname{tmp}(j-j 3)=\operatorname{bd}(6$, is $) * \operatorname{bd}(63$, is $)$

200

continue

if (ioout.eq.1) then

call uncnvt ('vl', tmp, j2-j3,1,-1)

endif

write $($ iout, 170) (ia $(1 j u n j+j-1), \operatorname{tmp}(j-j 0), \operatorname{tmp}(j-j 3), j=j 1, j 2)$

210 continue

write(iout, 220) (lumf, $i=1,8$ )

220

format//16x, 'junction mass flows (negative in to the plenum'.

2

do $240 i=1, i i$

' cell and positive out of the plenum cell $)^{\prime} / 4(8 x$,

$j 0=(i-1) * 4$

$j 1=j 0+1$ 
$j 2=\min 0(j 1+3, \operatorname{npljn})$

$j 3=j 0+j 0-j 2$

do $230 j=j 1, j 2$

$\operatorname{tmp}(j-j 0)=a(1$ rmvm $+j-1)-a(1$ rvm $f+j-1)$

$\operatorname{tmp}(j-j 3)=a(1 r v m f+j-1)$

continue

if (ioout.eq.1) then

call uncnvt ('rmvm', tmp, $j 2-j 3,1,-1)$

endif

write (iout, 170) (ia $(1 j u n j+j-1), \operatorname{tmp}(j-j 0), \operatorname{tmp}(j-j 3), j=j 1, j 2)$

240 continue

endif

C

cfmass $=v o l(k) *(\operatorname{arv}(k)+\operatorname{arl}(k))$

cener $=\operatorname{vol}(k) *(\operatorname{arev}(k)+\operatorname{arel}(k))$

omass $=$ cfmass - bsmass

$\operatorname{tmp}(1)=$ cfmass

$\operatorname{tmp}(2)=$ omass

$\operatorname{tmp}(3)=$ cener

if (ioout.eq.1) then

call uncnvt ('cfmass', tmp (1), $2,1,-i$ )

call uncnvt ('cener' , tmp (3), $1,1,-1)$

endif

write (iout, 250) tmp (1), lum, tmp (3), lue, tmp (2), lum

250 format $(/ '$ plenum component total coolant mass is',1p,e13.5,

$1 \quad a, \cdot$, total coolant energy is', e13.5,a,', and'/

2 ' computed initial total coolant mass is',e13.5,a)

c

return

end

\section{Fortran file wprizr.f:}

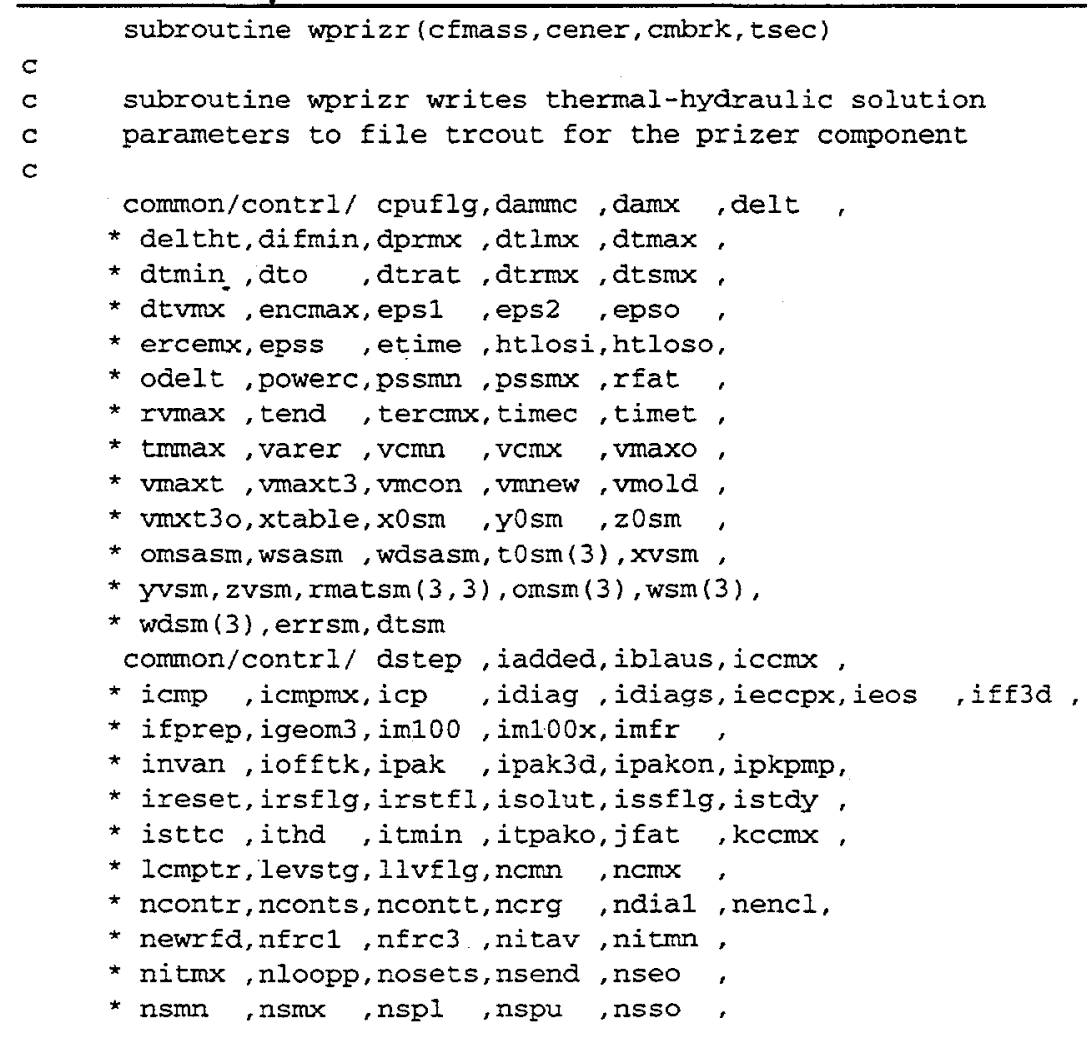


* nstab ,nstp ,nvgrav, nvpow , oitmax, sitmax,

* stdyst, transi,motsm, statsm, saxsm

integer dstep, oitmax, sitmax, stdyst.

* transi, statsm, saxsm

C

common/units/ ibfadd,ibfadg, ibfadr,

* ibflnd, ibflng, ibflnr, idout, ieeeg, igout ,

* ikeybd, imout, in ,inlab, inproc.

* ioall, iodone, ioerr , iogrf , ioinp.

* iolab , ioout, ioskip, iout, irstrt.

* itty , iunlab, iunout, icmcpd, nittab.

* npwtab, nrdy

common/cunits/card

character $* 100$ card

comon/istat/ varerm, verr

common/istat/ iott, nstep, oitno

integer oitno

common/labelv/factor (150), offset (150)

common/labelv/ih (26), itls (777), itsv(105),

ils, ilu, ilun, iold

common/labelv/labels (777), labsv (2, 105), labun (150),

$1 \quad \operatorname{Iuncb}(2,150), \operatorname{lupcb}(2,150)$, runcb (2, 150)

common/labelv/alpbet, luar, Iucp, lud, ludh, lue, Iuen.

1 Iuh, Iuha, Iuhx, Iuid, Iuis, Ium, Iumf, Iup,

2 Iupd, Iuph, lupt, Iupw, Iur, Ius, Iusp, Iusz ,

3 Iut, lutc, lutm, lutp, luv, luvf, luvo, Iuz

character*2 lud, lutp, lus

character*3 lut, Iuz

character $* 4$ luar, lue, lum, luvo

character*5 lup, Iupd, Iuv, Iuvf

character*6 luis

character 7 Iuen, Iumf, lupt, lupw

character $* 8$ labels, labun, luid, lur, lusp, lusz, lutm

character*9 luha

character*10 Iucp

character ${ }^{11}$ luph

character $* 12$ luhx, lutc, runcb

character $* 13$ luh, Iuncb, Iupcb

- character ${ }^{14}$ labsv

character*19 ludh

character $* 26$ alpbet

parameter

(ifxsiz $=5000000$ )

common a(ifxsiz)

dimension alcm(1)

equivalence (a(1), alcm(1))

dimension ia (ifxsiz)

character $* 8$ ach(ifxsiz)

equivalence (a(1), $a c h(1))$

equivalence (a(1), ia(1))

fixed length table

integer $f l t(1)$

real rfit (1)

equivalence (fIt (1), rfIt (I))

character type*8,title*32, ctitle*8

common/fltab/ htlsci,htlsco, coild, pinteg

common/fltab/ ctitle(4),icflg, id

* irest , lenarr, lenfv, lenfv2, lenptr,

* lenvit, lextra, Ifv , lfvn, ncelit.

* numbra1, numbom2, numbm3, numbn1, numbn2. 
* numbn3, nodes, num ,nrvit, ctype

equivalence (flt(1), htlsci), (type, ctype), (ctitle(1), title)

c

c prizer variable length table

dimension $f I(2), f v(2)$

common/vitab/aal111, bsmass, bsmssp, bxmass,

* dpmax , epsw ,fl ,flow, fv , houtl,

* houtv, pset, qheat, qin , qint.

* qout, gp 3 in, radin, th, tout .

* toutv, z ,zhts, z11111

common/vitab/ ial1ll,ichf, icj, iconc,

* ict1, iuv1 ,iuv2 ,js1,js2,

* jun1, jun2, ncells, type1.

* type2,

* zi1111

character*8 type1, type2

integer vit, zil11I

c-----comdeck vltdim sets an upper limit to all variable length tables

c----via parameter ivitsz. comdeck genvlt calis vltdim directly, thus

c----providing all variable length tables with a uniform length.

c----parameter ivitsz is used to set a uniform length to all

c----variable length tables.

parameter (ivltsz $=270$ )

dimension vlt(ivitsz)

equivalence (vIt(I), aa1I11)

$\mathrm{c}$

dimension $1 d(3), 1 d n(3)$

common/ptab/ lalp, lalpn, lalpd,

* lalpdn, lalv , lalvn, lalve, lalven,

* lara , Iaran , larel , lareln, larev.

* larevn, larl, larln, larv, larvn.

* lbit , Ibitn, lchti, Ichtin, Ichtia.

* Ichtan, lcif , lcifn, lcone, Iconen.

* la , Ian, lea, lean, lel

* leln, lev, levn, Igam, Igamn.

* Ihig, Ihigo, Ihil , Ihilo, Ihiv,

* Inivo . Ip Ipn , Ipa Ipan .

* Igppc, Iqppco, Iroa , Iroan, Irol .

* Iroln, irov, Irovn, is , Isn.

* Itce. Itcen, Ita , Itan, itl.

* Itlr. itv , Itva , itw , Itwa.

* Itwa , Itwan, Itwe , Itwen, Ivl IvIn,

* Ivlt, Ivlto , Ivm , Ivmn , Ivv

* Ivvr , Ivvt I Ivvto

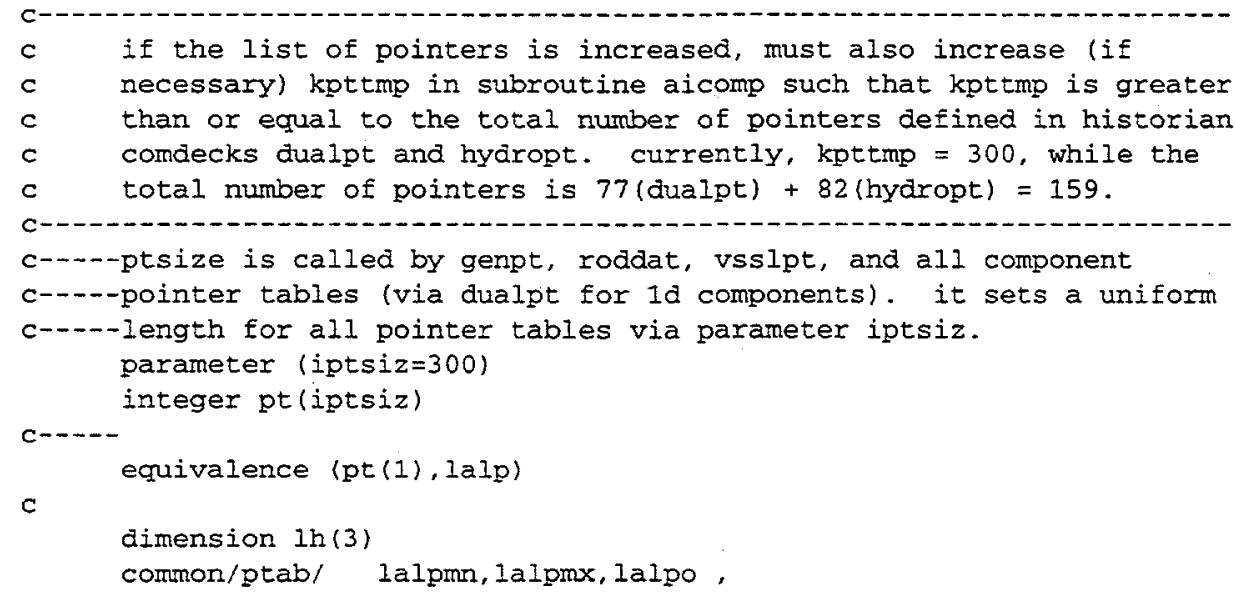


* lam , larc, lcfz, lcl, lcpl

* lcpv , lcv , ldalva, ldfldp, ldfvap.

* Idriv .Idx , Ielev, Ifa , Ifavol.

* Ifinan, Ifric, Ifsmit, Igrav, Igrvol,

* Ih ,Ihd, Ihdht, Ihfg, Ihgam.

* Inla , Ihlatw, Ihva ,Ihvatw,

* Iqri , Iqrv, Iqp $3 \mathrm{f}$.

* Iqppp , Iregnm,Irhs , Irmem, Irmvm ,

* Irarl , Irarv,

* Ixsm, Iysm, Izsm, Irsm, Irosm,

* Infvsm, Inflsm, luvsm, Infcvsm, Infclsm, 1vvsm, 1vlsm,

* $\operatorname{lnf} 1 \mathrm{sm}, \operatorname{lnf} 2 \mathrm{sm}, \operatorname{lnf} 3 \mathrm{sm}, \operatorname{lnfv} 4 \mathrm{sm}, \operatorname{lnf} 14 \mathrm{sm}$,

* Irom , Irvnf, Isig , Itrid, ltsat.

* Itssn , lvisl , Ivisv, Ivlalp, Ivlve,

* Ivlvol,ivlx , Ivol , lvr , lvrv

* Ivvrol, Ivvx , Iwa , lwat, lwfi.

* Iwfv , Iwfmfl, Iwfmfv

$c$

- dimension tmp (4)

$c$

$c$

$c$

if the list of pointers is increased, must also increase (if necessary) kpttmp in subroutine aicomp such that kpttmp is greater than or equal to the total number of pointers defined in historian comdecks dualpt and hydropt. currently, kpttmp $=300$, while the total number of pointers is 77 (dualpt) +82 (hydropt) $=159$.

in case new pointers are added after lwfmfv, then the coding in subroutine aicomp (third executable statement) for evaluation of the local variable lenpt $1=$ locf (Iwfmfv) - locf $(p t(1)$ ) 1 must be changed to use the name of the new pointer at the end of this section of the common block / ptab /.

\section{common/ptab/ liar, Imatid, lnff, llccfl}

common/ptab/ lcpw, 1cw, 10r

* Iemis, thol, Ihov, Irn, Irn2

* Irow, 1tchf, Itol, Itov

write(iout, 100) num, jun1, jun2

100 format $/ /$ the component type is a prizer, component number is',

1 i4,', Eirst junction number is', i4, ' and second'.

2 'junction number is', i4)

cImass $=0.0$

cener $=0.0$

c

$i \pm 1 g w=1$

itee $=0$

call ecomp (num, 1, ncells, iflgw, nodes, cfmass, pinteg, cener, tsec,

$\&$ itee)

if a steady-state calculation was or is being evaluated prior to a transient calculation, the mass checker must account for the increase in the volume of the component by a factor of $1.0 e+35$ during the steady-state calculation. This is done by assigning the fraction $1.0 e-35$ of the junction mass flow during the steady-state calculation to the component itself and the remainder to the total break flow; this logic assumes that a steady-state calculation may only be run before a transient 
c

if (stayst.eq.0) then

omass $=$ cfmass - bsmssp $-1.0 e-35^{\star}$ bsmass

else

qout $=1.0 e-35^{\star}$ qout

cfmass $=1.0 e-35^{*}$ cfmass

cener $=1.0 \mathrm{e}-35^{\star}$ cener

omass $=$ cfmass $-1.0 e-35 *$ bsmass

endif

c

$\operatorname{tmp}(1)=$ flow

$\operatorname{tmp}(2)=$ qout

$\operatorname{tmp}(3)=\operatorname{amax} 1(0.0, z)$

$\operatorname{tmp}(4)=q i n$

if (ioout.eq.1) then

call uncnvt ('flow', tmp (1), 1, 1, -1)

call uncnvt ('gout' , $\operatorname{tmp}(2), 1,1,-1)$

call uncnvt (' $z$ ' , tmp (3), $1,1,-1$ )

call uncnvt ('qin' , tmp (4), $1,1,-1)$ endif

write (iout, 110) tmp (1), Iuvf, tmp (2), Iuvo, tmp (3), Iuz, tmp (4), lupw

110 format $/ /$ prizer Iiquid volumetric outflow is',1p, e13.5,a,

1 ', volume out is',e13.5,a,', and level is',e13.5,a//

2 'prizer heater/sprayer power is',e13.5,a)

c

if (nodes.ne.0) and. (nstep.gt.0)) then

$\operatorname{tmp}(1)=h t I s c i$

$\operatorname{tmp}(2)=h t$ lsco

if (ioout.eq.1) then

call uncnvt('htlsci', tmp $, 2,1,-1)$

endif

write(iout, 120) tmp(1), lue, tmp (2), lue

120 format (" prizer wall total power loss on the inside',

1 ' surface is', 1p,e13.5,a,' and on the outside',

2 ' surface is', e13.5,a)

endif

c

cmbrk=bsmass

$\operatorname{tmp}(1)=$ cfmass

$\operatorname{tmp}(2)=$ omass

$\operatorname{tinp}(3)=$ cener

if (ioout.eq.1) then

call unenvt ('cfmass', tmp (1), 2, 1, -1)

call uncnvt ('cener' , tmp $(3), 1,1,-1)$ endif

write (iout, 130) tmp (1), lum, tmp (3), lue, tmp (2), lum

130 format (/" prizer component total coolant mass is',1p,e13.5,

1

2

a, ', total coolant energy is', e13.5,a,', and'/

' computed initial total coolant mass is',e13.5,a)

$c$

return

end

\section{Fortran file wpump.f:}

subroutine wpump (cfmass, cener, tsec)

$c$

subroutine wpump writes thermal-hydraulic solution

parameters to file trcout for the pump component

parameter

(ifxsiz $=5000000$ ) 
common a(ifxsiz)

dimension alcm(I)

equivalence $(a(1), \operatorname{alcm}(1))$

dimension ia (ifxsiz)

character*8 ach (ifxsiz)

equivalence (a(1),ach(I))

equivalence (a(1), ia(1))

c

c fixed length table

integer fIt (1)

real rflt(1)

equivalence ( $f \mathrm{lt}(1), \mathrm{r} f \mathrm{It}(1))$

character type 8, title ${ }^{\star} 32$, ctitle* 8

common/fltab/ htlsci,htlsco,coild, pinteg

comon/fltab/ ctitle(4),icflg, id

* irest, lenarr, lenfv, lenfv2, lenptr.

* lenvlt, lextra, lfv , Ifvn , ncelit.

* numbm1, numbm2, numbm3, numbn1, numbon2.

* numbn3, nodes , num , nrvit, ctype

equivalence (flt (1), htlsci), (type, ctype), (ctitle(1), title)

$c$

comon/units/ ibfadd, ibfadg, ibfadr,

* ibflna, ibflng, ibflnr, idout, ieeeg, igout.

* ikeybo,imout, in ,inlab, inproc.

* ioall, iodone, ioerr, iogrf, ioinp.

* iolab, ioout , ioskip, iout ,irstrt.

* itty , iunlab, iunout, lcmcpd, nittab.

* npwtab, nrdy

common/cunits/card

character $* 100$ card

c

common/istat/ varerm, verr

common/istat/ iott, nstep, oitno

integer oitno

C

common/labelv/factor (150), offset (150)

common/labelv/ih(26), itls (777), itsv(105),

1 ils, ilu, ilun, iold

common/labelv/labels (777), Iabsv (2, 105), labun (150),

1

luncb $(2,150), \operatorname{lupcb}(2,150), \operatorname{runcb}(2,150)$

- common/labelv/alpbet, luar, lucp, lud, ludh, lue, luen,

1

Iuh, Iuha, Iuhx, Iuid, Iuis, Ium, lumf, lup,

2

3

character* 2 lud, lutp, Ius

character*3 Iut, Iuz

character*4 luar, Iue, Ium, luvo

character*5 lup, lupd, Iuv, Iuvf

character*6 luis

character*7 luen, lumf, lupt, Iupw

character*8 labels, labun, luid, lur, lusp, Iusz, Iutm

character*9 Iuha

character*10 lucp

character*11 Iuph

character*12 Iubx, lutc, runcb

character ${ }^{\star 13}$ luh, luncb, lupcb

character*14 labsv

character*19 ludh

character $* 26$ alpbet

c

pump variable length table

dimension $f l(2)$, $f v(2)$

common/vitab/ aall11, aeffmi, alpha, 
* alphao, beffmi, bsmass, ceffmi, delp

* dsmom, effmi , effmil, epsw, fl flow,

* fv head, houtl, houtv , mflow.

* omega ,omegan, omgoff, omtest, gp 3 in,

* gp3off,radin, rflow, rhead, tho

* romega, romgmx, rqp $3 \mathrm{mx}$, rrho , rtork .

* smom, tfro, tfrl, tfr2, tfr3.

* tfrb , tfxlo, tfrl1, tfri2, tfri3,

* th , torque, toutl, toutv.

* z11111

dimension ndata(16)

common/vltab/ ial111,ichf, icj1, icj2,

* iconc, indxhm, indxtm, ionoff, ipf

* ipm ,ipmps, ipmps2, ipmpsv, ipmptr.

* ipmpty,ipp , iqf , iqp , iqp3sv,

* iqp 3 tr,irp , isol1, isol2, js1

* js2 ,jun1 ,jun2 ,ncells,ndata.

* ndmax , nhdin , nonoff, nompre, npmpsd.

* npmpsv, npmptb, nqp 3 rf, nqp3 sv, nqp3 tb.

* ntam , option, type1, type2 .

* zi1111

integer vit, typel, type2, zill11, option

real mflow

c-----comdeck vltdim sets an upper limit to all variable length tables

c-----via parameter ivltsz. comdeck genvlt calls vltdim directly, thus

c-..-providing all variable length tables with a uniform length.

c-..--parameter ivitsz is used to set a uniform length to all

c----variable length tables.

parameter (ivitsz $=270$ )

dimension vlt(ivltsz)

equivalence (vlt(1), aa1111)

c--n

$c$

c

dimension tmp (8)

$c$

write(iout, 100) num, jun1, jun2

100 format (/" the component type is a pump, component number is',

1 i4,', first junction number is', i4,', and second',

2 'junction number is', i4)

write(iout, 110)

110 format (/" trip',4x, 'impeller', 5x, 'liquid', 5x, 'volumetric', 6x,

1 'mass', $8 x$, 'void', 6x, 'momentum', 7x, 'pump' , $8 x$, 'delta', $8 x$

'pump'/' status' , 5x, 'speed',6x, 'density', 7x, 'flow', 9x,

'flow', $6 \mathrm{x}$, 'fraction', 5x, 'source', $8 \mathrm{x}$, 'head', 7x, 'pressure',

4 if (iunout.eq. 1 ) then

write (iout, 120) luis, Iur, Iuvf, lumf, lud, luph, luph, Iupd, lupt

120 format $(11 x, a, 5 x, a, 6 x, a, 7 x, a, 8 x, a, 5 x, a, 2 x, a, 5 x, a, 6 x, a)$

endif

iset $=0$

if (ipmptr.ne.0) call trip(ipmptr, iset, tlaps, delsv)

tmp $(I)=$ omegan

$\operatorname{tmp}(2)=r h o$

$\operatorname{tmp}(3)=$ flow

tmp $(4)=$ mflow

tmp $(5)=$ smom

$\operatorname{tmp}(5)=$ head

$\operatorname{tmp}(7)=\operatorname{delp}$

$\operatorname{tmp}(8)=$ tarque

if (ioout.eq.1) then 
call uncnvt ('omegan' $\operatorname{tmp}(1), 1,1,-1$ )

call uncnvt('rhol' ,tmp (2), $1,1,-1$ )

call uncnvt ('rflow' , tmp (3), 1, 1, -1)

call uncnvt ('mflow' , $\operatorname{tmp}(4), 1,1,-1)$

cal1 uncnvt ('smom' ,tmp $(5), 1,1,-1$ )

call uncnvt ('head' , tmp $(5), 1,1,-1)$

call uncnvt ('deltap', tmp $(7), 1,1,-1$ )

call uncnvt ('torque', tmp $(8), 1,1,-1)$

endif

write (iout, 130) iset, ( $\operatorname{tmp}(i), i=1,4)$, alpha, (tmp (i), $i=5,8)$

130 format (i5, 1p, el4.4, 3e13.5, 0p, £10.6,1p,4e13.5)

c

cfmass $=0.0$

cener $=0.0$

$c$

iflgw $=1$

itee $=0$

call ecomp (num, 1, ncells, iflgw, nodes, cfmass, pinteg, cener, tsec,

$\&$

itee)

if ((nodes.ne.0) and. (nstep.gt.0)) then

$\operatorname{tmp}(1)=h t$ lsci

$\operatorname{tmp}(2)=h t 1 s c o$

if (ioout.eq.1) then

call uncnvt('htlsci', tmp, 2,1,-1)

endif

write (iout, 140) tmp (1), lupw, tmp (2), lupw

140 format (/' pump wall total power loss on the inner surface is',

$1 \quad 1 p, e 13.5, a,{ }^{\prime}$ and on the outer surface is',e13.5,a)

endif

$c$

Omass $=$ cfmass - bsmass

$\operatorname{tmp}(1)=$ cfmass

tmp $(2)=$ omass

$\operatorname{tmp}(3)=$ cener

if (ioout.eq.1) then

cal1 uncnvt ('cfmass', tmp (1), $2,1,-1$ )

call uncnvt ('cener' , tmp (3), $1,1,-1$ )

endif

write (iout, 150) tmp (1), Ium, tmp (3), Iue, tmp (2), Ium

150 format $/ /$ pump component total coolant mass is',1p,e13.5,

1 a,' , total coolant energy is', el3.5,a,', and'/

2 ' computed initial total coolant mass is', e13.5,a)

c

return

end

\section{Fortran file wtee.f:}

subroutine wtee (cfmass, cener, tsec)

c

subroutine wtee writes thermal-hydraulic solution

parameters to file trcout for the tee component

common/contrl/ cpuflg, damme, damx, delt,

* deltht, difmin, aprmx , dtlmx , dtmax ,

* atmin ,ato , dtrat ,atrmx , dtsmx ,

* dtvmx , encmax, eps 1 , eps 2 ,epso.

* ercemx, epss , etime ,htlosi,htloso,

* odelt, powerc,pssmn ,pssmx , rfat

* rvmax , tend , tercmx, timec , timet.

* tmmax , varer, vcmn , vcrux , vmaxo, 
* vmaxt , vmaxt3, vmcon, vmnew, vmold,

* vinxt3o,xtable, x0sm,y0sm, z0sm,

* omsasm, wsasm , wdsasm, tosm(3), xvsm ,

* yvsm, zvsm, rmatsm( $(3,3), \operatorname{omsm}(3), \operatorname{wsm}(3)$.

* wdsm (3), errsm, dtsm

common/contrl/ dstep, iadded, iblaus, iccmx ,

* icmp, icmprn, icp, idiag, idiags, ieccpx, ieos, iff3d,

* ifprep, igeom 3, im100, im100x, imfr

* invan , iofftk, ipak ,ipak3d,ipakon, ipkpmp,

* ireset, irsflg, irstfl, isolut, issflg, istdy ,

* isttc, ithd , itmin, itpako, jfat, kccmx ,

* Icmptr, levstg, Ilvflg, ncmn, ncmx ,

* ncontr, nconts, ncontt, ncrg , ndial , nencl,

* newrfa, nfrc1, nfrc3, nitav , nitmn ,

* nitmx , nloopp, nosets, nsend, nseo ,

* nsmr , nsmx ,nspl , nspu , nsso

* nstab , nstp , nvgrav, nvpow , oitmax, sitmax,

* stdyst, transi, motsm, statsm, saxsm

integer dstep , oitmax, sitmax, stdyst,

* transi, statsm, saxsm

c

common/holl/ accumh, breakh, coreh,

* ctainh, fillh, pipeh ,plenh ,prizrh,pumph .

* stgenh, teeh , turbh, valveh, vssih

* ,rodh, slabh, sepdh character $* 8$ rodh, slabh, accumh, breakh, coreh, ctainh, fillh, pipeh

1, plenh, prizrh, pumph, stgenh, teeh, turbh, valveh, vssih

$c$

* sepdh

common/units/ ibfadd, ibfadg,ibfadr,

* ibflnd, ibflng, ibflnr, idout, ieeeg, igout.

* ikeybd, imout, in , inlab, inproc,

* ioall, iođone, ioerr, iogrf, ioinp,

* iolab, ioout, ioskip, iout , irstrt,

* itty , iunlab, iunout, lcmepd, nittab.

* npwtab, nrdy common/cunits/card

character $* 100$ card

c

common/istat/ varerm, verr

commonistat/ iott, nstep, oitno

integer oitno

$c$

common/labelv/factor (150), offset (150)

common/labelv/ih (26), itls (777), itsv(105).

1

ils, ilu, ilun, iold

common/labelv/labels (777), labsv (2, 105), labun (150),

1

luncb $(2,150), \operatorname{Iupcb}(2,150), \operatorname{runcb}(2,150)$

common/labelv/alpbet, luar, Iucp, Iud, ludh, lue, luen,

1 Iuh, Iuha, Iuhx, Iuid, Iuis, Ium, Iumf, Iup,

2 lupd, luph, lupt, lupw, lur, Ius, 1usp, Iusz,

3 Iut, Iutc, Iutm, Iutp, Iuv, Iuvf, luvo, Iuz

character*2 lud, lutp, Ius

character $* 3$ lut, luz

character*4 luar, lue, lum, luvo

character*5 lup, lupd, luv, luvf

character* 6 luis

character*7 luen, Iumf, lupt, lupw

character*8 labels, labun, Iuid, Iur, Iusp, Iusz, Iutm

character $* 9$ luha

character*10 lucp

character*11 luph 
character $* 12$ luhx, Iutc, runcb

character*13 luh, luncb, lupcb

character*14 labsv

character*19 ludh

character $* 26$ alpbet

parameter (ifxsiz=5000000)

common a (ifxsiz)

dimension alcm(1)

equivalence (a(1), alcm(1))

dimension ia (ifxsiz)

character $* 8$ ach (ifxsiz)

equivalence (a(1), $\operatorname{ach}(1))$

equivalence $(a(1)$, ia(1))

c

c fixed length table

integer $f I t(I)$

real rflt (1)

equivalence ( $f(t)(1), \operatorname{rflt}(1)$ )

character type*8,title*32, ctitle*8

common/fltab/ htlsci,htlsco,coild, pinteg

common/fltab/ ctitle(4),icflg, id

* irest, lenarr, lenfv, lenfv2, lenptr,

* lenvlt, lextra, Ifv , Ifvn , ncelit,

* numbm1, numbrn2, numbom3, numba1, numbn2,

* numbri, nodes, num , nrvit, ctype

equivalence (flt (1), htlsci), (type, ctype), (ctitle(1), title)

c

$\mathrm{c}$

c tee variable length table

dimension $f I(4), f v(4)$

common/vitab/ aal11, ai ,alpot,alpotn,alpoto,alpd ,

* alps ,an ,bsmass, ca ,cal

* calv , cav , cost, deldim, dpsep,

* dpss ,epsw , enin1, enin2 .

* fl ,fv ,houtl1, hout12, houtv1,

* houtv2, powr1 , powr2, pwinl ,pwin2,

* pwofe1, pwoff2, qpin1, qpin2 , qpoff1,

* qpoff2, radin1, radin2, rh ,rpwmx1,

* rpwmx2, rqprnx1, rqpmx2,

* rr1 , rt1 , rt1v, rt2l, rt2v

* thi , th2 , theta, tlen1, tlen2,

* toutl1, tout 12, toutv1, toutv2, varyl ,

* vdryu , wlio ,xco ,xcu ,

* z11111

common/vitab/ ial111,icbs1, icbs2,

* ichf , icj1, icj2, icj3, iconcl,

* iconc2,iary, ientrn,ionof1, ionof2,

* ipf1 ,ipf2, ipow1, ipow2 , ipp1,

* ipp2 ,ipwsv1, ipwsv2, ipwtr1, ipwtr2,

* iqf1,iqf2, iqp1,iqp2, iqpsv1,

* iqpsv2, iqptr1, iqptr2, isep , isol1,

* isol2, isol3, isoln, istage,jcell,

* js1 ,js2 ,js3,

* jun1 , jun2, jun3 ", ncel11,

* ncel12, ncel1s, ncsep , ndryr , nonof1,

* nonof2, npwrf1, npwrf2, npwsv1, npwsv2,

* npwtb1, npwtb2, nqprf1, nqpr 22, nqpsv1,

* nqpsv2, nqptb1, nqptb2, nseps , type1 ,

* type 2 , type3

* zi1111

integer vit,type1, type2, type , zi1111

c-----comdeck vitdim sets an upper limit to all variable length tables 
c-----via parameter ivltsz. comdeck genvlt calls vltdim directly, thus c----providing all variable length tables with a uniform length.

c----parameter ivitsz is used to set a uniform length to all

c----variable length tables.

parameter (ivltsz=270)

dimension vit(ivltsz)

equivalence (vit (1), aal111)

$c-\cdots-$

c tee pointer table

dimension $\operatorname{ld}(3), \operatorname{lon}(3)$

common/ptab/ lalp, lalpn, lalpd,

* lalpdn, lalv, lalvn, lalve , lalven,

* lara , laran, larel , Iareln, larev,

* larevn, larl, larln , larv , larvn,

* lbit , loitn, lchti , Ichtin, lchtia,

* lchtan, lcif, Icifn, Icone, Iconen,

* la , lar , lea , Iean , lel

* lein, lev, levn, Igam, Igamn,

* Ihig, Ihigo, Ihil , Ihilo, Ihiv

* Ihivo, ip , Ipn , Ipa ,Ipan

* lappe, Igppco, Iroa , Iroan, Irol

* Iroln, irov, Irovn, is , isn

* Itce, Itcen, Itd, Itan, it

* Itin, itv, Itvn, itw, itwn

* Itwa , Itwan, Itwe , Itwen, Ivi , Ivin,

* Ivlt, ivito, Ivm, Ivmn, Ivv,

* Ivvn , Ivvt, Ivvto

$c$ if the list of pointers is increased, must aiso increase (if

c necessary) kpttmp in subroutine aicomp such that kpttmp is greater

c than or equal to the total number of pointers defined in historian

c comdecks dualpt and hydropt. currently, kpttmp $=300$, while the

c total number of pointers is 77 (dualpt) +82 (hydropt) $=159$.

c----ptsize is called by genpt, roddat, vsslpt, and all component

c----pointer tables (via dualpt for 1 d components). it sets a uniform

c----length for all pointer tables via parameter iptsiz.

parameter (iptsiz=300)

integer pt(iptsiz)

c.....-

equivalence (pt $(1), 1 a l p)$

c

dimension $1 \mathrm{~h}(3)$

common/ptab/ lalpmn, lalpmx, lalpo.

* lam , larc, lcfz, lcl , lcpl,

* lcpv, lcv , Idalva, Idfldp, Idfvdp,

* Idriv, ldx , lelev, Ifa , Ifavol,

* Ifinan, Ifric , Ifsmlt, Igrav, Igrvol,

* Ih , Ihd , Ihdht, Ihfg, Ihgam,

* Ihla , Ihlatw, Ihva ,Ihvatw.

* lqr 1 , Iqrv , lqp3e,

* lqppp, Iregnm, Irhs , Irmem, Irmvm

* Irarl , Irarv ,

* 1xsm, Iysm, Izsm, 1rsm, Irosm,

* Infvsm, Inflsm, luvsm, Infevsm, Infclsm, 1vvsm, 1vlsm,

* $\operatorname{lnf} 1 \mathrm{sm}, \operatorname{lnf} 2 \mathrm{sm}, 1 \mathrm{nf} 3 \mathrm{sm}, 1 \mathrm{nfv} 4 \mathrm{sm}, 1 \mathrm{nf} 14 \mathrm{sm}$,

* Irom , Irvme , Isig , ltrid, Itsat,

* Itssn, Ivisl, Ivisv, Ivialp, Ivlve,

* Ivivol, Ivlx . Ivol , Ivr ivrv,

* Ivvvol, ivvx , Iwa , lwat, IwfI,

* Iwfv. Iwfmel, Iwfmfv 





iflgw $=0$

itee $=2$

call ecomp (num, ncell1+2, ncells, iflgw, nodes, cfmass, pinteg, cener, $\&$ tsec, itee)

C

if (ipow2.eq.1) then

$\operatorname{tmp}(1)=$ powr 2

$\operatorname{tmp}(2)=$ enin2 + delt $t$ powr2

if (ioout.eq. I) then

call uncnvt ('powr2', tmp (1), $1,1,-1$ )

call uncnvt('enin2', $\operatorname{tmp}(2), 1,1,-1$ )

endif

write(iout, 140) tmp(1), lupw, tmp (2), lue

140 format (/" power deposited in the tee-secondary coolant is',

I Ip, e13.5,a, ' and total energy deposited in the tee-',

2 'secondary coolant is', e13.5,a)

endif

$c$

if (type.eq.sepdh) then

write(iout, 150) xco, xcu

150 format (/' tee separator carryover is',1p,e13.5.

1 ' and carryunder is', e13.5)

endif

c

if ((nodes.ne.0) and. (nstep.gt.0)) then

$\operatorname{tmp}(1)=h t 1 s c i$

$\operatorname{tmp}(2)=h t i s c o$

if (ioout.eq.1) then

call uncnvt ('helsci', tmp, $2,1,-1)$

endif

write (iout, 160) tmp (1), lupw, tmp (2), lupw

160 format (/' tee wall total power loss on the inner surface is',

1 1p,e13.5,a,' and on the outer surface is',e13.5,a)

endif

$c$

omass=cfmass-bsmass

$\operatorname{tmp}(1)=$ cfmass

$\operatorname{tmp}(2)=$ omass

$\operatorname{tmp}(3)=$ cener

if (ioout.eq.1) then

cal1. uncnvt ('cfmass', tmp (1), $2,1,-1$ )

call uncnvt ('cener', $\operatorname{tmp}(3), 1,1,-1)$

endif

write (iout, 170) tmp (1), Ium, tmp (3), lue, tmp (2), Ium

170 format $/ /$ tee component total coolant mass is',1p,e13.5.

1 a, ', total coolant energy is',el3.5,a, ', and'/

2 ' computed initial total coolant mass is',e13.5,a)

c

return

end

\section{Fortran file wturb.f:}

subroutine wturb (cfmass, cener, tsec)

$\mathrm{c}$

c subroutine wturb writes thermal-hydraulic solution

c parameters to file trcout for the turb component

c

common/contrl/ cpuflg, damme, damx , delt,

* deltht, difmin, dprmx , dt lmx ,dtmax ,

* atmin ,ato ,atrat atrmx , dtsmx .

* dtvmx ,encmax, eps1, eps2, epso, 
* ercemx, epss ,etime, htlosi,htloso,

* odelt, powerc, pssmn, pssmx, rfat

* rvmax , tend , tercmx, timec ,timet ,

* tmmax, varer, vcrin , vcmx , vmaxo,

* vmaxt, vmaxt3, vmcon, vmnew, vmold,

* vinxt3o, xtable, x0sm, yosm, z0sm

* omsasm, wsasm, wasasm, tosm (3), xvsm ,

* yvsm, zvsm, rmatsm $(3,3), \operatorname{omsm}(3), w \operatorname{wsm}(3)$,

* wdsm (3), errsm, dtsm

common/contrl/ dstep, iadded,iblaus, iccmx ,

* icmp, icmpmx, icp , idiag, idiags, ieccpx, ieos, iff3d,

* ifprep, igeom3, imi00,im100x,imfr

* invan, iofftk, ipak ,ipak3d, ipakon, ipkpmp,

* ireset, irsflg, irstfl, isolut, issflg, istdy .

* isttc, ithd , itmin, itpako, jfat , kccmx ,

* lcmptr, levstg, 11 vflg, ncmn , ncmx ,

* ncontr, nconts, ncontt, ncrg , ndial, nencl,

* newrfa, nfrc1 , nfrc3 , nitav, nitmn,

* nitmx , nloopp, nosets, nsend, nseo ,

* nsmr , nsmx , nspl inspu , nsso,

* nstab, nstp , nvgrav, nvpow , oitmax, sitmax,

* stdyst, transi,motsm, statsm, saxsm

integer dstep , oitmax, sitmax, stdyst,

* transi, statsm, saxsm

common/dimen/ ifree, jnvssl,kvel1t,

* kvel2t, kvel3t, last , ldim , lenbd, lendim,

* lentbl, Ifree , Ilast , Imldp , Inlapv, Inrapt,

* locrap, 1start, Istrdp, Iver ,maim,

* menf.Ig, moff , ncomp, ncompt.

* nhtstr, njnmx .njnt ,njun .nloops,

* nmvssi, npx ,nstgj , nthm , nthmia,

* nthm3a, numtcr, nvcon, nvelx , nvely,

* nvelz, nwrda

common/units/ ibfadd, ibfadg, ibfadr,

* ibflnd, ibflng, ibflnr, idout, ieeeg, igout .

* ikeybd, imout, in ,inlab, inproc,

* ioall, iodone, ioerr, iogrf ,ioinp,

* iolab, ioout, ioskip, iout , irstrt,

* itty , iunlab, iunout, lcmcpd, nittab,

* npwtab, nrdy

common /cunits/card

character $* 100$ card

c

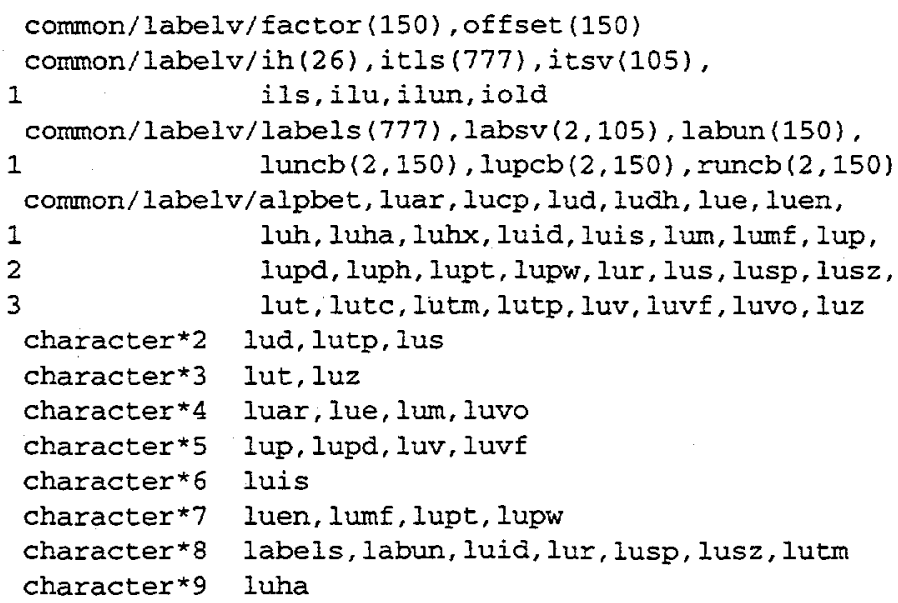


character*10 lucp

character $* 11$ luph

character*12 luhx, lutc, runcb

character*13 luh, luncb, lupcb

character $* 14$ labsv

character ${ }^{*} 19$ Iudh

character ${ }^{2} 6$ alpbet

parameter $\quad($ ifxsiz $=5000000)$

common a (ifxsiz)

dimension alcm(1)

equivalence (a(1), alcm(1))

dimension ia (ifxsiz)

character $* 8$ ach (ifxsiz)

equivalence (a(1), ach (1))

equivalence $(a(1)$, ia(1))

$c$

c fixed length table

integer fit (1)

real rflt(1)

equivalence (fIt (1), rflt(1))

character type* 8, title* 32 , ctitle*8

common/fltab/ htlsci,htisco,coild, pinteg

common/fltab/ ctitle(4), icflg, id

* irest, lenarr, Ienfv, lenfv2, lenptr.

* lenvlt, lextra, Ifv , lfvn, ncellt.

* numbom1, numbm2, numbm3, numbn1, numbn2,

* numbin, nodes, num , nrvlt, ctype

equivalence (flt ( 1 ), htlsci), (type, ctype), (ctitle(1), title)

$c$

c turbine stage variable length table

dimension $f l(2), f v(2)$

common/v1tab/ aal111, alpha1,alpha2,

* ar bsmass, coef1.

* coef2, cof3sq, cp, cpow, dia ,

* dsmom, effdsn, effstg, eninp, epsw , fl

* flodir, flow , fv gamma, phirem,plent.

* powin ,powoff, powdsn, powstg, pres1,

* pres2 ,qualty, reactn, rhol1, rhol2,

* rhom1, rhom2, rhov1, rhov2, rpowmx,

* smom .. suprht, temp11, templ2, tempv1,

* tempv2, vell1, vell2, velm1, velm2,

* velv1, velv2, vstag,

* $z 11111$

common/vltab/ ial111,icjl, icj2, iconc,

* ionoff,ipf ,ipow ,ipowsv, ipowtr.

* ipp ,isollb, isolrb, istg, jsl

* js2 ,jun1, jun2, lentxb, ncells,

* nefcon, npowrf, npowsv, npowtb, nrows .

* type1 , type2,

* zi1111 integer vit, type1, type2,zil111

c-----comdeck vltdim sets an upper limit to all variable length tables

c-----via parameter ivltsz. comdeck genvit calls vltdim directly, thus

c----providing all variable length tables with a uniform length.

c----parameter ivitsz is used to set a uniform length to all

c----variable length tables.

parameter (iv1tsz=270)

dimension vit (ivltsz)

equivalence (vIt(1), aal111)

$c$ 
turbine stage pointer table

dimension $1 d(3), 1 d n(3)$

common/ptab/ lalp, lalpn, lalpd.

* lalpdn, lalv , lalvn, lalve, laiven.

* lara , laran, larel , lareln, larev.

* larevn, larl , larln , larv , larvn,

* lbit Ibitn, Ichti , Ichtin, Ichtia,

* Ichtan, lcif , lcifn, Icone, lconcn,

* la , lan, lea , lean, lel

* leln , lev , levn, Igam, Igamn,

* Ihig, Ihigo, Ihil , Ihilo, Ihiv

* Ihivo, lp ,Ipn , Ipa ,lpan

* loppc, Iqppco, Iroa , Iroan, Irol

* Iroln, Irov , Irovn, is , Isn

* Itce, Itcen, Ita , itan, Itl

* Itln , ltv , Itvn , Itw , Itwn

* ltwa , Itwan, Itwe , Itwen, Ivl , lvin,

* Ivlt, ivito. Ivm, lvmn, ivv,

* Ivvr , Ivvt Ivvto

$c$ if the list of pointers is increased, must also increase (if

c necessary) kpttmp in subroutine aicomp such that kpttmp is greater

c than or equal to the total number of pointers defined in historian

c comdecks dualpt and hydropt. currently, kpttmp $=300$, while the

c total number of pointers is 77 (dualpt) +82 (hydropt) $=159$.

c---ptsize is called by genpt, roddat, vsslpt, and all component

c----pointer tables (via dualpt for Id components). It sets a uniform

c----length for all pointer tables via parameter iptsiz.

parameter (iptsiz=300)

integer pt(iptsiz)

c-----

equivalence (pt (1), lalp)

$c$

dimension $3 \mathrm{~h}(3)$

common/ptab/ lalpmn, lalpmx, lalpo,

* lam , larc, Icfz, Icl. , lcpl,

* lcpv, lcv , Idalva, ldfldp, Idfvdp,

* Iariv, Idx . Ielev, Ifa , Ifavol,

* Ifinan, Ifric . Ifsmlt, Igrav, Igrvol,

* Ih , lha . Ihdht, linfg, lingam,

* Ihla , lhlatw, Ihva , Ihvatw,

* lqri , lqxv , Iqp3f.

* Iqppp ,Iregnm, Irhs , Irmem, Irmvm ,

* Irarl, Irarv.

* 1xsm, Iysm, 1zsm, 1rsm, 1rosm,

* Infvsm, Inflsm, luvsm, Infcvsm, Infclsm, 1vvsm, Ivism,

* Inflsm, Inf2sm, lnf3sm, Infv4sm, Infl4sm,

* Irom , Irvmf Isig , Itrid, Itsat,

* Itssn , Ivisl , Ivisv , Ivlalp, lvive.

* Ivlvol, lvix, Ivol, lvr. Ivrv.

* Ivvrol, lvvx , Iwa , Iwat , lwfl,

* Iwfv , Iwfmfl, Iwfinfv

c

if the list of pointers is increased, must also increase (if

necessaryl kpttmp in subroutine aicomp such that kpttmp is greater than or equal to the total number of pointers defined in historian comdecks dualpt and hydropt. currently, kpttmp $=300$, while the total number of pointers is 77 (dualpt) +82 (hydropt) $=159$.

in case new pointers are added after lwfmfv, then the coding in 


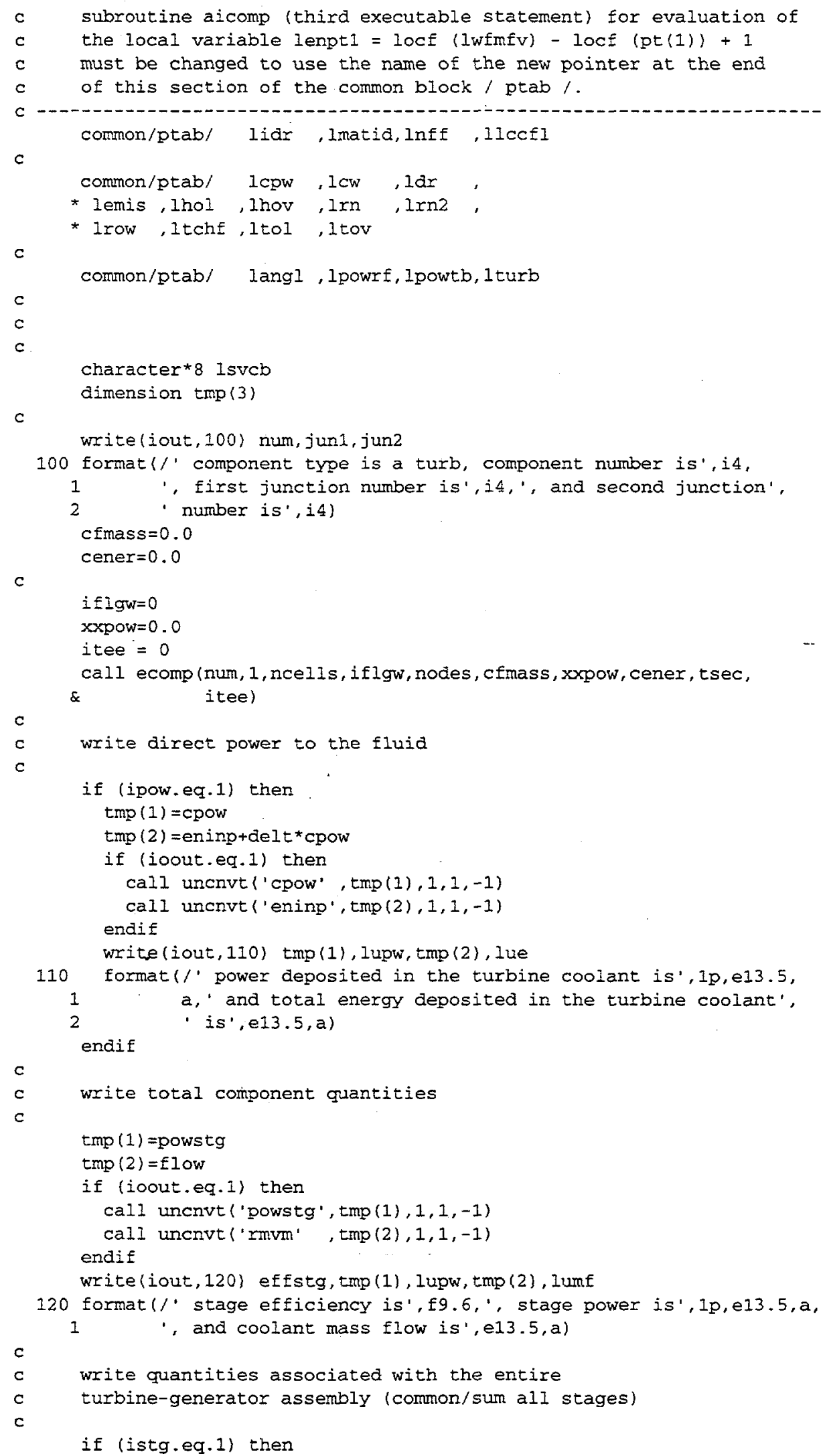


I turb=1 turb-ifree +1

$\operatorname{tmp}(1)=a($ ifree + lenarr +10$)$

tmp $(2)=a($ ifree + lenarr +12$)$

$\operatorname{tmp}(3)=a($ ifree + lenar $r+20)$

call unsvcb (itrpsv, lsvcb, iun)

if (ioout.eq.I) then

call uncnvt ('powd' , tmp $(1), 1,1,-1)$

call uncnvt ('omegd' , $\operatorname{tmp}(2), 1,1,-1)$

call uncnvt (Isvcb , tmp (3), $1,1,-1$ ) endif

write (iout, 130) tmp (1), Iupw, tmp(2), Iuis,

1 tmp (3), Iuncb (ioout+1, iun)

130 format//" the following quantities are associated with the', ' entire turbine assembly'/ generator power is',1p, e13.5,a, ', turbine speed is', e13.5,a,', and turbine', endif

' signal value is', e13.5, a)

$c$

omass $=$ cfmass - bsmass

$\operatorname{tmp}(1)=$ cfmass

$\operatorname{tmp}(2)=$ omass

$\operatorname{tmp}(3)=$ cener

if (ioout.eq.1) then

call uncnvt ('cfmass', $\operatorname{tmp}(1), 2,1,-1$ )

call uncnvt ('cener', tmp ( 3$), 1,1,-1$ )

endif

write (iout, 140) tmp (1), Ium, tmp (3), lue, tmp (2), ium

140 format (/' turb component total coolant mass is',1p,e13.5,

1 a,', total coolant energy is', e13.5,a,', and'

2 ' computed initial total coolant mass is', el3.5,a)

c

return

end

\section{Fortran file wvlve.f:}

subroutine wvlve (cfmass, cener, tsec)

$c$

c subroutine wvlve writes thermal-hydraulic solution

c parameters to file trcout for the valve component

$c$.

parameter (ifxsiz=5000000)

common a (ifxsiz)

dimension alcm(I)

equivalence $(a(1), \operatorname{alcm}(1))$

dimension ia(ifxsiz)

character*8 ach(ifxsiz)

equivalence (a(1), $\operatorname{ach}(1))$

equivalence (a(1), ia(1))

$c$

c fixed length table

integer flt(I)

real $\operatorname{rfit}(1)$

equivalence (flt(i), rflt(I))

character type*8,title*32, ctitle*8

comon/fltab/ htlsci,htlsco,coild, pinteg

common/fltab/ ctitle(4),icflg, id

* irest , lenarr, lenfv. lenfv2, lenptr.

* lenvit, lextra, Ifv , Ifvn, ncellt,

* numbm1, numbm2, numbm3, numbn1, numbon2,

* numbn3,nodes, num ,nrvit, ctype

equivalence (flt (1), htlsci), (type, ctype), (ctitle(1), title) 
c

common/units/ ibfadd, ibfadg, ibfadr,

* ibflnd, ibflng, ibflnr, idout, ieeeg, igout,

* ikeybd, imout, in , inlab, inproc,

* ioall, iodone, ioerr, iogrf, ioinp,

* iolab, ioout, ioskip, iout, irstrt,

* itty, iunlab, iunout, lcmcpd, nittab,

* npwtab, nrdy

common/cunits/card

character $\star 100$ card

$c$

common/istat/ varerm, verr

common/istat/ iott, nstep, oitno

$c$

integer oitno

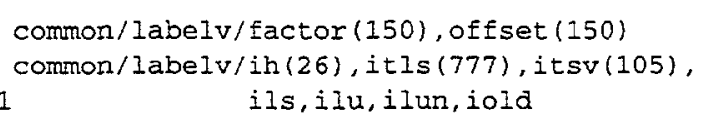


parameter $($ ivltsz $=270)$

dimension vlt(ivltsz)

equivalence (vlt (1), aal111)

c

vive pointer table

dimension $1 d(3), I d n(3)$

common/ptab/ lalp , lalpn, lalpd.

* lalpdn, lalv , lalvn , lalve , lalven,

* lara , laran , larel , lareln, larev.

* larevn, larl , larln , larv , larvn.

* Ibit , Ibitn, Ichti , Ichtin, Ichtia,

* Ichtan,lcif , lcifn, Icone, Iconen,

* la , lan , lea , lean , lel

* leln , lev, levn, igam, Igamn.

* Ihig, Ihigo, Ihil , Ihilo, Ihiv

* Ihivo, Ip , lpn , Ipa ,Ipan.

* Iqppc , Ioppco, iroa , Iroan irol.

* Iroln, irov, lrovn, is , Isn

* Itce, itcen, Ita , Itan, ItI

* Itln , Itv, Itvn, ltw , ltwn

* Itwa , Itwan, Itwe , Itwen, IvI , Ivln

* lvit, Ivlto, Ivm, lvmn, Ivv,

* Ivvr , lvvt , Ivvto

$c$ if the list of pointers is increased, must also increase (if necessary) kpttmp in subroutine aicomp such that kpttmp is greater than or equal to the total number of pointers defined in historian comdecks dualpt and hydropt. currently, kpttmp $=300$, while the total number of pointers is 77 (dualpt) +82 (hydropt) $=159$.

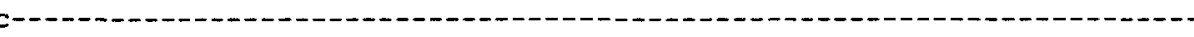

c----ptsize is called by genpt, roddat, vsslpt, and all component

c-----pointer tables (via dualpt for $1 d$ components). it sets a uniform

c----length for all pointer tables via parameter iptsiz. parameter (iptsiz=300)

integer pt (iptsiz)

c-o-

equivalence (pt (1), lalp)

$c$

dimension $1 \mathrm{~h}(3)$

- common/ptab/ lalpmn,lalpmx, lalpo.

* lam , larc, lefz, lcl , lcpl,

* lcpv , lcv , ldalva, laflap. Idfvap.

* Iariv, lax , lelev, lfa . Ifavol.

* Ifinan, Ifric , Ifsmit, lgrav , lgrvol,

* Ih , ind, lhdht, infg, Ihgam,

* Ihla , Ihlatw, lhva , Ihvatw,

* lqrl , Iqrv, , lqp3

* lqppp ,Iregnm, Irhs ,Irmem, Irmum ,

* Irarl irarv.

* Ixsm, lysm, lzsm, lrsm, lrosm,

* Infvsm, Inflsm, luvsm, Infcvsm, Infclsm, lvvsm, Ivlsm,

* Inf1sm, lnf2sm, lnf3sm, lnfv4sm, Infl4sm,

* Irom, Irvmf, Isig , Itrid, Itsat,

* Itssn, Ivisl , lvisv, Ivlalp, Ivlvc ,

* Ivlvol,ivix , Ivol , Ivr , Ivrv.

* Ivvvol, Ivvx , lwa , Iwat .Iwfl,

* Iwfv , Iwfmfl, Iwfmifv

$\dot{c}$

if the list of pointers is increased, must also increase (if necessary) kpttmp in subroutine aicomp such that kpttmp is greater 


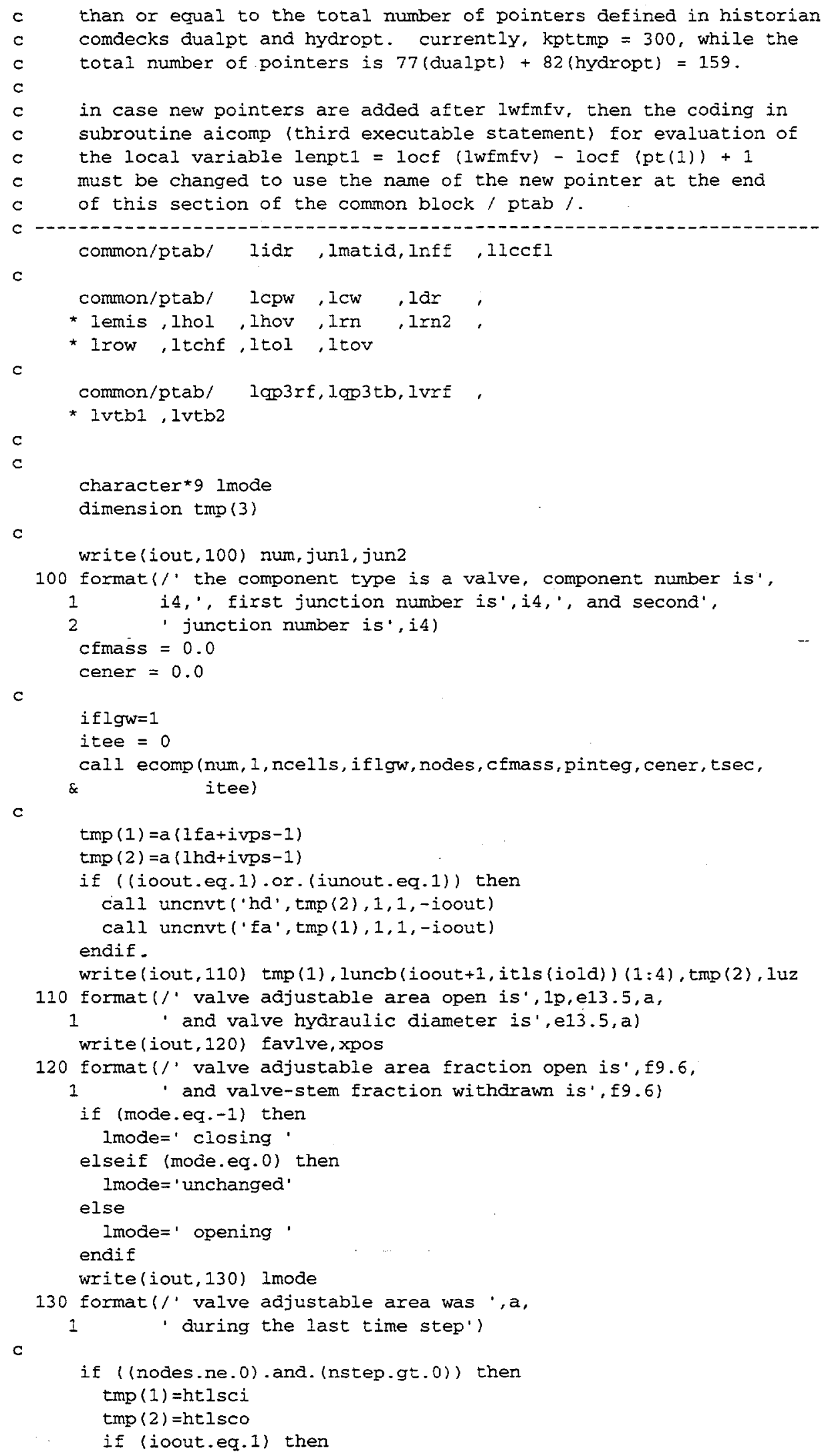




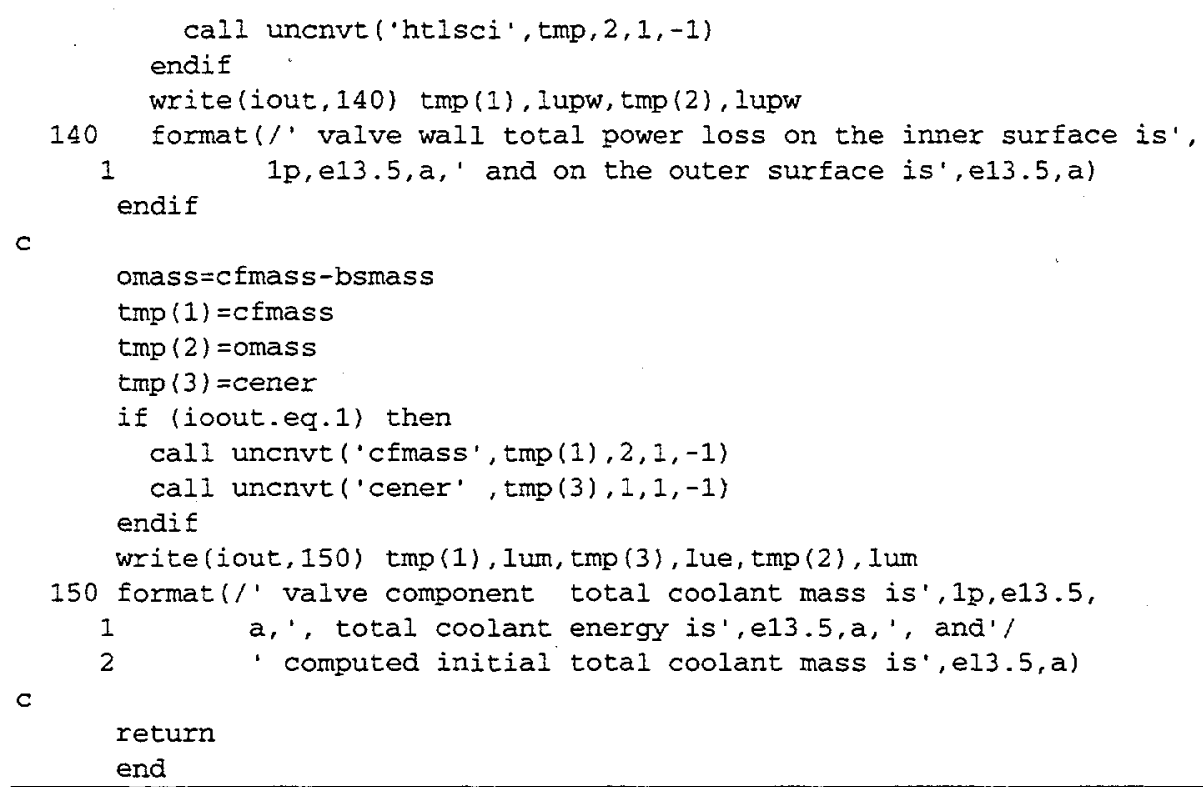




\section{Appendix B: Listing of Post-Processing Program TSPLIT}

The file listed below represents the entire source code for the TSPLIT algorithm. TSPLIT contains only a main program with no subroutines.

\section{Fortran file tsplit.f:}

program tsplit

$c$

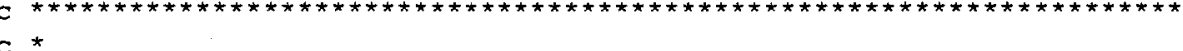

* Program "tsplit" takes as input the TRAC code generated

* "tecsum.grf" file and splits it out into separate "tecplot" files

* consistent with the requested graphics points inputted to TRAC

thru the "graphin" file.

* First the data within the "tecsum.grf" file is read in and stored

* within appropriate arrays consistent with component type. Then,

* each individual component graph point data set is written to its

own unique file (see output below).

INPUT: "graphin" file

"tecsum.grf" file

INTERMEDIATE:

OUTPUT:

$c *$

"tec???-xx.dat" files for graphics points in components that are not plenums or heat structures.

"tecp???-xx.dat" files for graphics points in plenum components.

"tecphi???-xx.dat" files for graphics points on inner surfaces of heat structures.

where ??? - represents TRAC component number

$x x$ - represents cell location within component

graphin arrays:

npoints - number of locations (points) within TRAC model where graphics requested

component - component id number containing specified graph point

cell - cell number within component where graphics requested (number second leg of tee sequentially from end cell number on first leg)

(If a heat structure then cell number is axial row of nodes where point resides)

ictype - type of component:

(0 for fill, pipe, pressurizer, pump, turb, value)

(1) for plenum)

(2) for tee)

(3 for heat structure)

$c$ * id_leg - which leg of component cell is located in:

$c *$

(1 always for fill, pipe, pressurizer, pump. 


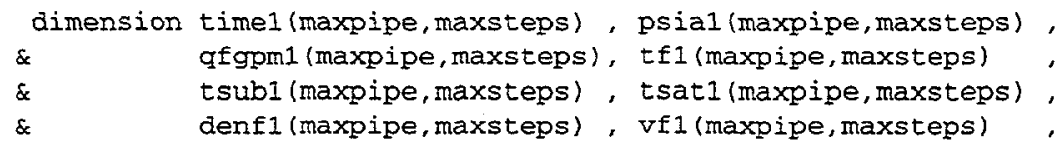




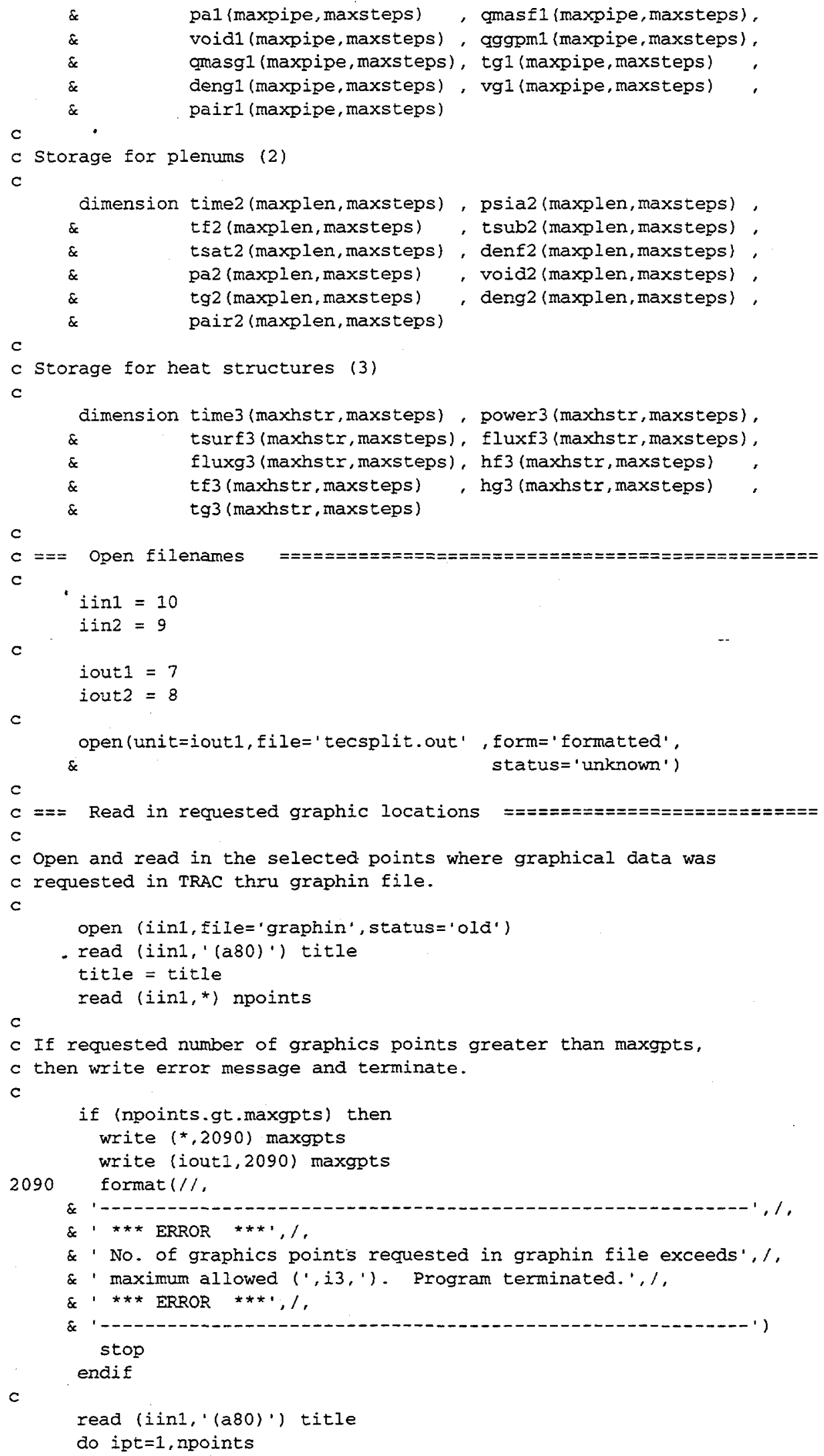


read (iinl,*) iicomp(ipt), iicell(ipt), ictype(ipt), id_leg(ipt)

c

cc write $(*, 2200)$

write (iout 1,2200 )

2200 format $(/)$ \& ' -

\& ' graphics point request file to TRAC opened: "graphin",/,

\& '

$c$

c Adjust cell id up one when component is a tee to be consistent

$c$ with storage scheme used in TRAC when cell is in the second

c leg of that tee.

c

do ipt $=1$, npoints

if (ictype (ipt).eq.2 and. ia_leg(ipt) .eq.2) then iicell $(i p t)=i$ icell $(i p t)+1$

endif

endao

$c$

c $===$ Open tecplot summary graphic data sets $========================$

$c$

c open summary tecplot graphics file lone file will contain all

c component graphics; external routine "tecsplit" is requred

$c$ to split out the graphics into multiple tecplot files for

c each requested graphics point):

$c$

Eilename $=$ 'tecsum.grf'

open (iin2, file=filename, status=' unknown')

cc write $(*, 2000)$

write (iout 1,2000 )

2000 format $(/)$,

\& '-..-

\& ' Tecplot graphics summary file opened: "tecsum.grf", 1 ,

c

\& ' '

$c===$ Extract from tecsum.grf file indexing $===============+=========0$

$\mathrm{C}$

C TRAC writes out the results for each requested graphics point in a

$c$ potentially different order that originally requested. Extract out

$c$ the ordering (indexing) from the first time step data set and then

$c$ rewind file for followon reading.

c

write (ioutl, '(/,"file unit indexing for components", $/)$ )

read (iin2,' (a80)') title

do ipt $=1$, npoints

read (iin2,*) jpt

index $(i p t)=j p t$

write (iout1.1101) ipt, jpt enddo

1101

format $(2 x, \cdot$ TRAC no. ', i3,'

graphin no. $=', i 31$

c

rewind (iin2)

read (iin2,'(a80)') title

$c$

$c$ check indexing for repeated numbers and terminate if found.

c

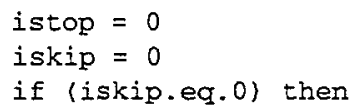


write (iout1, '(/,"Checking for repeated graphic points", /)')

do ipt $=1$, npoints

do jpt=1, npoints

if (ipt.ne.jpt) then

if (index(ipt).eq.index(jpt)) then

write (iout1,1201) ipt,jpt

1201 format (' graphic point ', i3,' repeated at graphic',

$\&$ ' point ',i3,/,'ERROR program terminated')

$$
\text { istop }=1
$$

endif

endif

$$
\text { endao }
$$

endio

$\mathrm{C}$

c check indexing for missing number and terminate if found.

$\mathrm{C}$

write (iout), (/,"Checking for missing graphic points",/)')

do ipt=1, npoints

icheck (ipt) $=0$

endido

do ipt $=1$, npoints

jpt $\quad=$ index (ipt)

$i \operatorname{check}(j p t)=1$

enddo

do ipt $=1$, npoints

if (icheck(ipt).eq.0) then

write (iout1,1211) ipt

1211 format (' graphic point ', i3,' missing',

$\&$

/. 'ERROR program terminated')

write (iout1,1221) iicomp(ipt), iicell(ipt), ictype(ipt),

$\&$

1221

$\&$ id_leg (ipt)

$\&$

format (' iicomp $=$ ', i3,' iicel $=$ ', i2,' ictype $=$ ', i2, istop $=1$

id_leg $=$, i2)

endif

endio

endif

c

if (istop.ne.0) stop

$c===$ Read each graphic point data to storage arays $==================$

$c$.

c Read in each graphic point for each time data set and store its

$c$ data into the appropriately defined storage arrays.

c

itime $=0$

8000

continue

ipipe $=0$

iplen $=0$

instr $=0$

itime $=$ itime +

c If requested number of time steps greater than maxgpts-1,

$c$ then write error message and terminate.

c

if (itime.gt.maxgpts-1) then

write $(*, 2092)$ maxgpts-1

write (iout1, 2092) maxgpts-1

2092

format $(/)$

$\&$ \& $\star \star \star * *$ ERROR $\star \star \star *, /$,

- No. of time step data sets in tecsum.grf file exceeds', /.

$\&$ ' maximum allowed $\left(", i 3,{ }^{\prime}\right)$. Program terminated. ', , 
$\& \cdot * *$ ERROR $* * * 1,1$,

\& 1 stop

endif

$c$

do ipt $=1$, npoints

$j p t=i n d e x(i p t)$

$c$

if (ictype (jpt).eq.0 .or. ictype(jpt).eq.2) then

c Read data if component is not a plenum component or heat structure.

C

ipipe = ipipe +1

read (iin2, *, end $=9000$ ) idum,

$\approx \quad$ timel(ipipe,itime) , psial(ipipe,itime),

\& $\quad$ qfgpml(ipipe,itime), tf1(ipipe,itime)

$\therefore \quad$ tsubl (ipipe,itime), tsatl(ipipe,itime),

\& denf1(ipipe,itime), vfl(ipipe,itime)

\& pal(ipipe,itime) , gmasfl(ipipe,itime),

\& voidl(ipipe, itime), gggpml (ipipe, itime),

\& qmasg1(ipipe,itime), tg1(ipipe,itime)

\& dengl(ipipe,itime), vgl(ipipe,itime)

idum $=$ idum

pairl (ipipe, itime)

C

c Read data if component is a plenum component.

$\mathrm{C}$

elseif (ictype(jpt).eq.1) then

iplen $=$ iplen +1

read (iin2, *, end $=9000$ ) idum,

time2(iplen,itime), psia2(iplen,itime) ,

tf2(iplen,itime), tsub2(iplen,itime),

tsat2 (iplen, itime), denf2(iplen, itime) ,

pa2(iplen,itime) , void2(iplen,itime) ,

tg2(iplen,itime) , deng2(iplen,itime) ,

pair2 (iplen, itime)

c Read data if component is a heat structure.

C

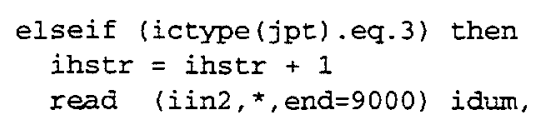


c For each graphic point requested in graphin file open file setting

c its filename consistent with requested graphic point's component

$c$ and cell location. Write out a header to file. Write graphic

$c$ point's time data to this opened

c file and then close file. Loop over each graphic point and perform

$c$ the above operations.

c

write (iout1, 2001)

2001 format (//,' Tecplot graphics Eilenames/unit numbers: './. $\&$

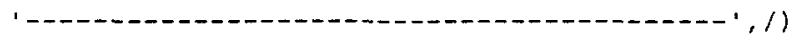

ipipe $=0$

iplen $=0$

ihstr $=0$

do ipt=1, npoints

jpt $=$ index $(i p t)$

if (ictype (jpt) .eq. 0 .or. ictype (jpt).eq.2) then

c Write data if component is not a plenum component or heat structure.

c Open file and write header:

c

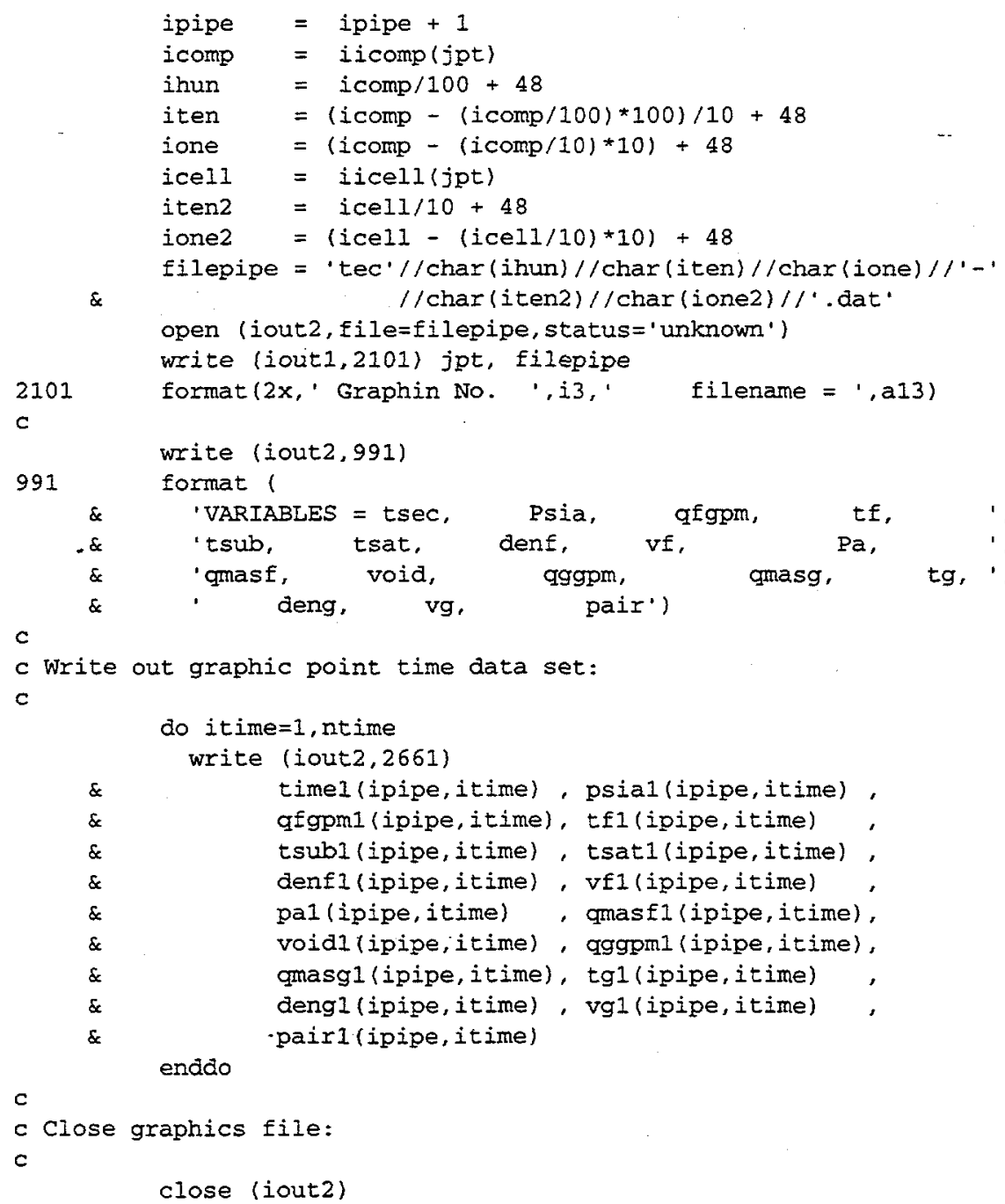


elseif (ictype(jpt).eq.1) then

$c$

c Write data if component is a plenum component.

$\mathrm{c}$

c Open file and write header:

$c$

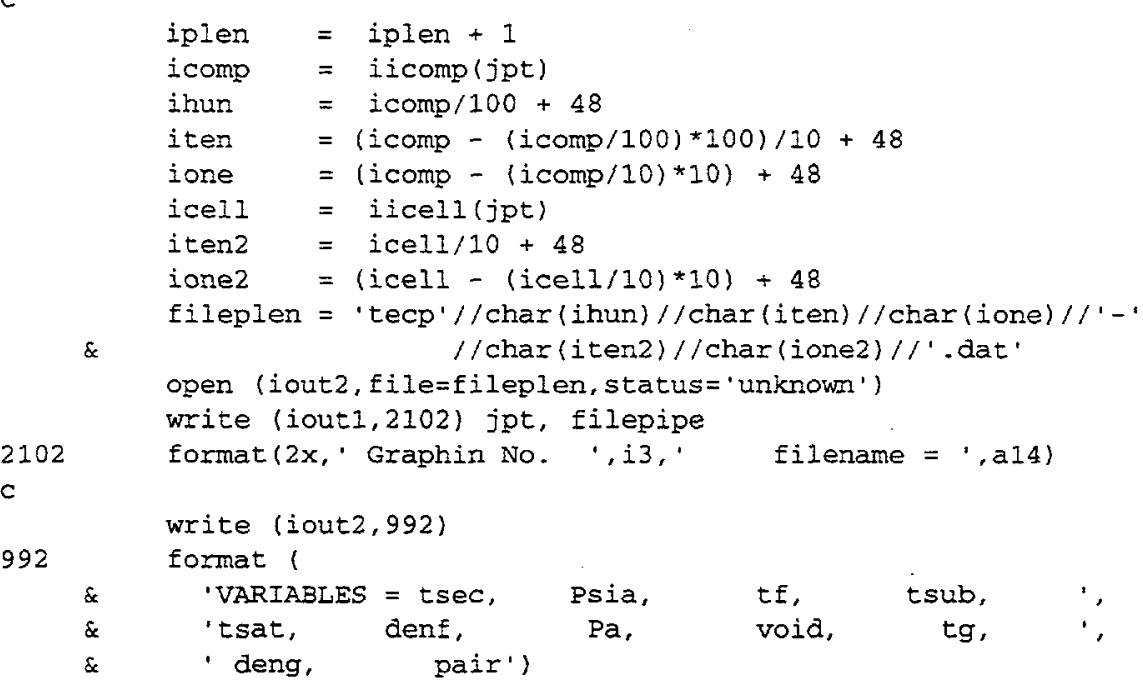

c Write out graphic point time data set:

$c$

do itime $=1$, ntime

write (iout2, 2662)

time2(iplen,itime) , psia2(iplen,itime),

tf2(iplen,itime), tsub2(iplen,itime),

tsat2(iplen,itime), denf2(iplen,itime),

pa2(iplen,itime) , void2(iplen,itime),

tg2(iplen, itime), deng2(iplen,itime),

enddo

pair2 (iplen, itime)

c

c Close graphics file:

c

close (iout2)

elseif (ictype(jpt).eq.3) then

$c$

$c$ Write data if component is a heat structure.

$c$

c Open file and write header:

c

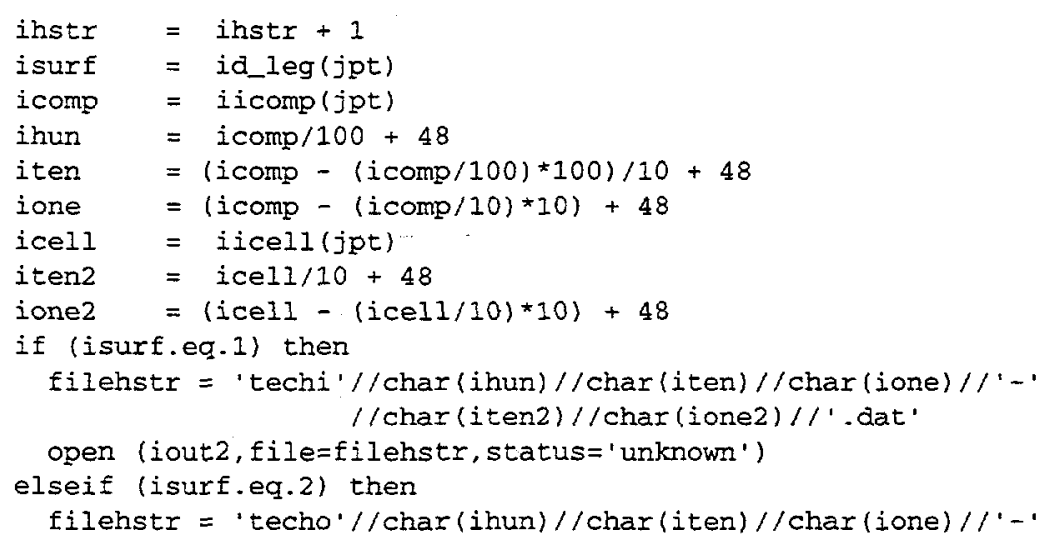


open (iout2, file=filehstr, status = 'unknown' ) endif write (iout1,2103) jpt, filepipe

2103

c

993 format $\{2 x$, ' Graphin No. ',i3,'

\&

c Write out graphic point time data set:

c

do itime $=1$, ntime

write (iout 2,2663 ) time3 (ihstr, itime), power3 (ihstr,itime), tsurf3(ihstr, itime), fluxf3 (ihstr, itime), fluxg3 (ihstr, itime), hf3 (ihstr, itime) tf 3 (ihstr,itime) , hg3(ihstr, itime) endio

c Close graphics file: endif enddo

$c$

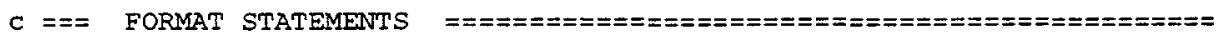
c

2661 format $(8 x, f 8.2, f 10.2, f 12.3,4 f 10.2, f 10.4, f 13.1, f 12.3, f 10.4$, el 4.4 , $\&$

$\mathrm{f} 12.3, f 10.2,2 \mp 10.4, f 10.2)$

2662 format $(8 \mathrm{x}, \mathrm{f} 8.2,5 \mathrm{f} 10.2, \mathrm{f} 13.1, f 10.4, f 10.2, f 10.4, f 10.2)$

2663 format $(8 \mathrm{x}, \mathrm{f} 8.2, \mathrm{e} 14.5, \mathrm{f} 10.2,2 \mathrm{e} 14.5, \mathrm{f} 12.3, \mathrm{f} 10.2, \mathrm{f} 12.3, \mathrm{f} 10.2)$

c

stop

end 


\section{Appendix C: Listing of Example "graphin" File}

The file listed below represents an example "graphin" file typically used during a Blanket System accident analysis TRAC run. Also provide at the end of the file is a legend.

\section{Input file graphin:}

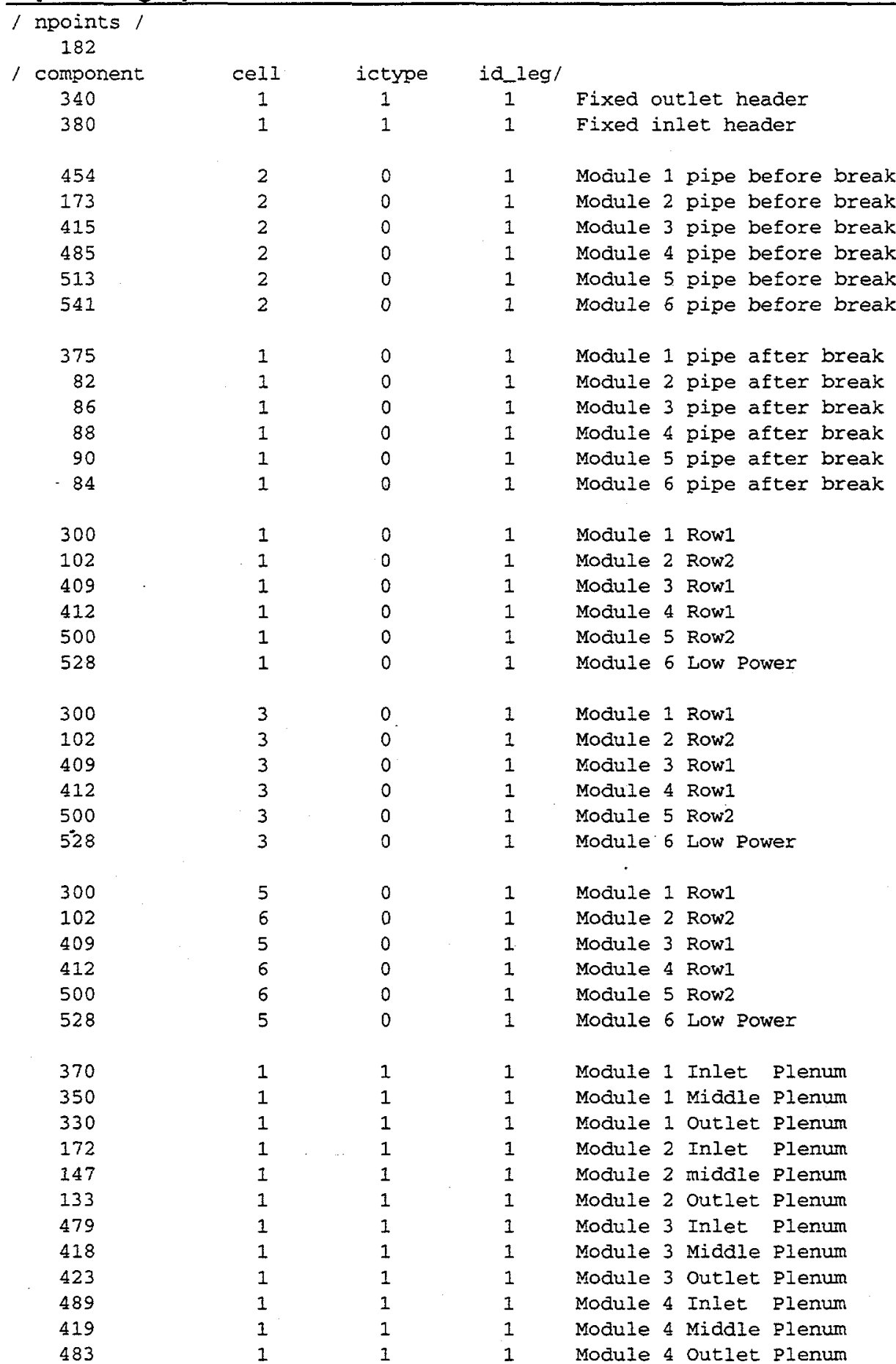




\section{0}

503

508

538

531

536

26

$\begin{array}{llll}1 & 1 & 1 \\ 1 & 1 & & 1 \\ 1 & 1 & & 1 \\ 1 & 1 & & 1 \\ 1 & 1 & 1 \\ 1 & 1 & & 1\end{array}$

1
1
1
1
1
1

1

\begin{abstract}
Module 5 Inlet Plenum Module 5 Midale plenum Module 5 outlet Plenum Module 6 Inlet Plenum Module 6 Midale Plenum Module 6 outlet Plenum
\end{abstract}

Hot leg pump suction line

Hot leg purn suction line near break Hot leg pump suction line near break

PCL Pump 1 suction

PCL Pump 1 Discharge

PCL Pump 2 Suction

PCL Pump 2 Discharge

PCL Pump 1 check valve

PCL Pump 2 check valve

PCL Pump to $\mathrm{HX}$

PCL $\mathrm{Fx} 1$ inlet

PCL Hx 1 middle

PCL $\mathrm{HX} 1$ outlet

PCL HX 2 inlet

PCL $\mathrm{Hx} 2$ middle

PCL $\mathrm{H} \times 2$ outlet

PCL HX 1 secondary side outlet

PCL $\mathrm{H} \times 1$ secondary side inlet fill

PCL HX 2 secondary side outlet

PCL HX 2 secondary side inlet fill

Cold leg $\mathrm{HX}$ discharge line

Cold leg $\mathrm{Hx}$ discharge line near break Cold leg Fix discharge line near break Cold leg $\mathrm{Hx}$ discharge line near break Cold leg $\mathrm{Hx}$ discharge line near break Cold leg $\mathrm{Hx}$ discharge line near break

RHR Pump Suction

RHR Pump Discharge

RHR $\mathrm{HX}$ inlet

RHR Hx outlet

RHR Hx secondary side outlet

RHR HX secondary side inlet fill

Pzr Pressure Signal

pzr Bottom pressure

Hot Module (1) upflow inside

Hot Module (1) upflow inside

Hot Module (1) upflow inside

Hot Module (1) upflow outside

Hot Module (1) upflow outside

Hot Moduie (1) upflow outside

Hot Module (2) upflow inside

Hot Module (2) upflow inside Hot Module (2) upflow inside Hot Module (2) upflow outside Hot Module (2) upflow outside Hot Module (2) upflow outside 


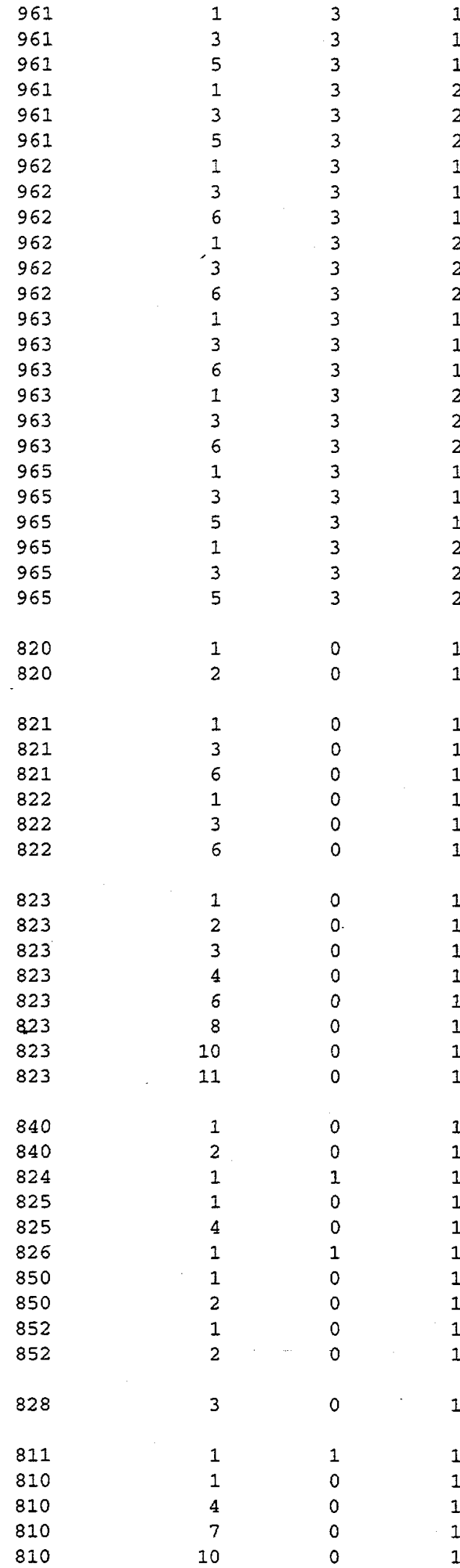

Hot Module (3) upflow inside Hot Module (3) upflow inside Hot Module (3) upflow outside Hot Module (3) upflow outside Hot Module (3) upflow outside Hot Module (3) upflow outside Hot Module (4) upflow inside Hot Module (4) upflow inside Hot Module (4) upflow inside Hot Module (4) upflow outside Hot Module (4) upflow outside Hot Module (4) upflow outside Hot Module (5) upflow inside Hot Module (5) upflow inside Hot Module (5) upflow inside Hot Module (5) upflow outside Hot Module (5) upflow outside Hot Module (5) upflow outside Hot Module (6) upflow inside Hot Module (6) upflow inside Hot Module (6) upflow inside Hot Module (6) upflow outside Hot Module (6) upflow outside Hot Module (6) upflow outside

Flood valve pool side

flood valve vessel side

Flood horizontal line

Flood horizontal line

Flood horizontal line

Flood vertical line

Flood vertical line

Flood vertical line

Cavity vessel lower 1 Cavity vessel lower 2 Cavity vessel lower 3 Cavity vessel lower 4 Cavity vessel lower 6 Cavity vessel lower 8 Cavity vessel lower 10 Cavity vessel lower 11

Cavity line to Hot module loc. I Cavity line to Hot module 10c. 2 Cavity vessel hot module loc. Cavity vessel middle 1

Cavity vessel middle 1

Cavity vessel HR line loc.

Cavity line to HR line (HR side)

Cavity line to HR line (HR side)

Cavity line to HR line (Header side)

Cavity line to HR line (Header side)

Vent Iine break side

Cavity Pool flood line plenum

Cavity pool upper 1

Cavity pool upper 4

Cavity pool upper 7

Cavity pool upper 10 


\section{4}

984

984

901

901

901

916

916

916

905

905

905

988

988

988

911

911

911

931

$$
931
$$$$
931
$$$$
912
$$$$
912
$$$$
912
$$$$
932
$$$$
932
$$$$
932
$$$$
913
$$$$
913
$$$$
913
$$

$\begin{array}{lll}1 & 3 & 2 \\ 3 & 3 & 2 \\ 5 & 3 & 2 \\ 1 & 3 & 2 \\ 3 & 3 & 2 \\ 5 & 3 & 2 \\ 1 & 3 & 2 \\ 3 & 3 & 2 \\ 6 & 3 & 2 \\ 1 & 3 & 2 \\ 3 & 3 & 2 \\ 6 & 3 & 2 \\ 1 & 3 & 2 \\ 3 & 3 & 2 \\ 5 & 3 & 2 \\ 1 & 3 & 2 \\ 3 & 3 & 2 \\ 5 & 3 & 2 \\ 1 & 3 & 2 \\ 3 & 3 & 2 \\ 6 & 3 & 2 \\ 1 & 3 & 2 \\ 3 & 3 & 2 \\ 6 & 3 & 2 \\ 1 & 3 & 2 \\ 3 & 3 & 2 \\ 6 & 3 & 2 \\ 1 & 3 & 2 \\ 3 & 3 & 2 \\ 6 & 3 & \end{array}$

Module 1 downflow Al 1

Module 1 downflow Al 3

Module 1 downflow Al 5

Module 1 upflow Al 1

Module 1 upflow AI 3

Module 1 upflow Al 5

Module 2 downflow AI 1

Module 2 downflow A1 3

Module 2 downflow Al 6

Module 2 upflow AI I

Module 2 upflow A1 3

Module 2 upflow Al 6

Module 3 downflow Al 1

Module 3 downflow Al 3

Module 3 downflow AI 5

Module 3 upflow Al 1

Module 3 upflow Al 3

Module 3 upflow Al 5

Module 4 downflow AI 1

Module 4 downflow AI 3

Module 4 downflow Al 6

Module 4 upflow Al 1

Module 4 upflow Al 3

Module 4 upfiow Al 6

Module 5 downflow Al 1

Module 5 downflow Al 3

Module 5 downflow AI 6

Module 5 upflow Al 1

Module 5 upflow Al 3

Module 5 upflow Al 6

INPUT NOTES:

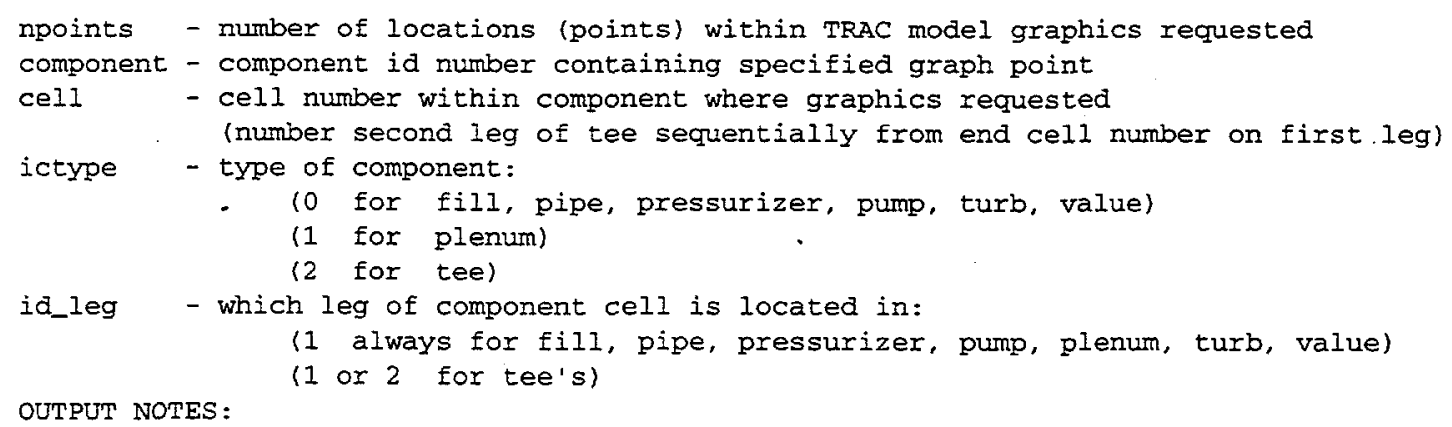

OUTPUT NOTES:

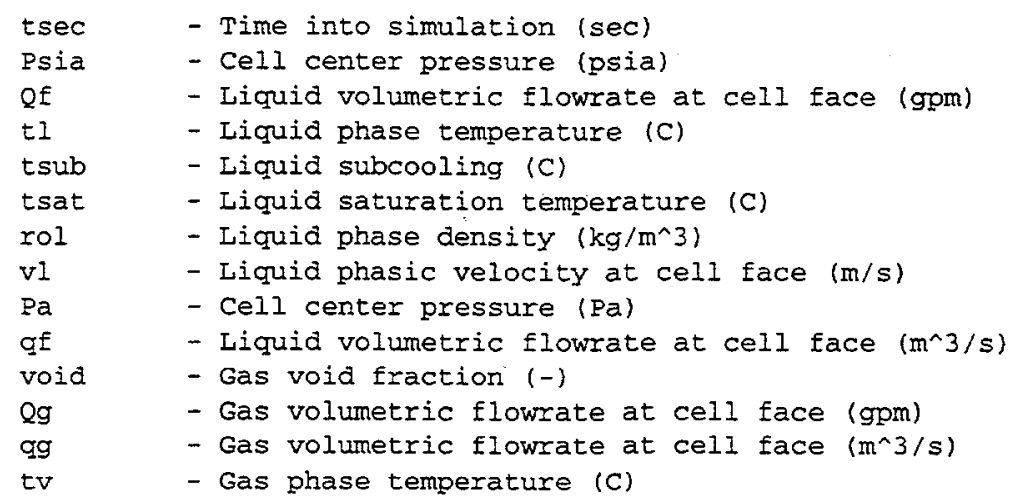


